

\section{EDITORIAL BOARD}

ANTONIO CARCATERRA ERIC A. CARLEN

FRANCESCO DELL'ISOLA

RAFFAELE ESPOSITO

ALBERT FANNJIANG

Gilles A. FrancFort

PiERANGElo MARCATI

JEAN-JACQUES MARIGO

PETER A. MARKOWICH MARTIN OSTOJA-STARZEWSKI

PIERRE SEPPECHER

DAVID J. STEIGMANN

PAUl STEINMANN

PierRe M. Suquet

\section{MANAGING EDITORS}

MICOL AMAR

CORRADO LATTANZIO

ANGELA MADEO

MARTIN OSTOJA-STARZEWSKI

\section{ADVISORY BOARD}

ADNAN AKAY

Holm AltenbaCH

MICOL AMAR

HARM ASKES

TEODOR ATANACKOVIĆ

VICTOR BERDICHEVSKY

GuY BouchitTÉ

ANDREA BRAIDES

ROBERTO CAMASSA

MAURO CARFORE

ERIC DARVE

FELIX DARVE

ANNA DE MASI

Gianpietro Del Piero

EMMANUELE Di BENEDETTO

BERNOLD FIEDLER

IRENE M. GAMBA

SERGEY GAVRILYUK

TIMOTHY J. HEALEY

DOMINIQUE JEULIN

ROGER E. KHAYAT

CORRADO LATTANZIO

ROBERT P. LIPTON

ANGELO LUONGO

ANGEla MadeO

JUAN J. MANFREDI

CARLO MARCHIORO

GÉrard A. MAUGin

ROBERTO NATALINI

PATRIZIO NEFF

ANDREY PIATNITSKI

ERrico Presutti

MARIO PUlVIRENTI

LuCiO RuSSO

Miguel A. F. SANJUAN

Patrick SElvadurai

ALEXANDER P. SEYRANIAN

MIROSLAV ŠILHAVÝ

GUIDO SWEERS

ANTOINETTE TORDESILLAS

LEV TRUSKINOVSKY

JUAN J. L. VELÁZQUEZ

VINCENZO VESPRI

ANGELO VULPIANI msp.org/memocs

Università di Roma “La Sapienza”, Italia

Rutgers University, USA

(CO-CHAIR) Università di Roma "La Sapienza", Italia

(TREASURER) Università dell'Aquila, Italia

University of California at Davis, USA

(CO-CHAIR) Université Paris-Nord, France

Università dell' Aquila, Italy

École Polytechnique, France

DAMTP Cambridge, UK, and University of Vienna, Austria

(CHAIR MANAGING EDITOR) Univ. of Illinois at Urbana-Champaign, USA

Université du Sud Toulon-Var, France

University of California at Berkeley, USA

Universität Erlangen-Nürnberg, Germany

LMA CNRS Marseille, France

Università di Roma “La Sapienza”, Italia

Università dell' Aquila, Italy

Université de Lyon-INSA (Institut National des Sciences Appliquées), France (CHAIR MANAGING EDITOR) Univ. of Illinois at Urbana-Champaign, USA

Carnegie Mellon University, USA, and Bilkent University, Turkey

Otto-von-Guericke-Universität Magdeburg, Germany

Università di Roma "La Sapienza”, Italia

University of Sheffield, UK

University of Novi Sad, Serbia

Wayne State University, USA

Université du Sud Toulon-Var, France

Università di Roma Tor Vergata, Italia

University of North Carolina at Chapel Hill, USA

Università di Pavia, Italia

Stanford University, USA

Institut Polytechnique de Grenoble, France

Università dell'Aquila, Italia

Università di Ferrara and International Research Center MEMOCS, Italia

Vanderbilt University, USA

Freie Universität Berlin, Germany

University of Texas at Austin, USA

Université Aix-Marseille, France

Cornell University, USA

École des Mines, France

University of Western Ontario, Canada

Università dell' Aquila, Italy

Louisiana State University, USA

Università dell' Aquila, Italia

Université de Lyon-INSA (Institut National des Sciences Appliquées), France

University of Pittsburgh, USA

Università di Roma "La Sapienza", Italia

Université Paris VI, France

Istituto per le Applicazioni del Calcolo "M. Picone", Italy

Universität Duisburg-Essen, Germany

Narvik University College, Norway, Russia

Università di Roma Tor Vergata, Italy

Università di Roma "La Sapienza", Italia

Università di Roma “Tor Vergata", Italia

Universidad Rey Juan Carlos, Madrid, Spain

McGill University, Canada

Moscow State Lomonosov University, Russia

Academy of Sciences of the Czech Republic

Universität zu Köln, Germany

University of Melbourne, Australia

École Polytechnique, France

Bonn University, Germany

Università di Firenze, Italia

Università di Roma La Sapienza, Italia

MEMOCS (ISSN 2325-3444 electronic, 2326-7186 printed) is a journal of the International Research Center for the Mathematics and Mechanics of Complex Systems at the Università dell'Aquila, Italy.

Cover image: "Tangle" by @ John Horigan; produced using the Context Free program (contextfreeart.org).

\section{PUBLISHED BY}

mathematical sciences publishers

nonprofit scientific publishing

http://msp.org/

(C) 2014 Mathematical Sciences Publishers 


\title{
DELAMINATED THIN ELASTIC INCLUSIONS INSIDE ELASTIC BODIES
}

\author{
Alexander M. KhludneV And GÜNTER R. LeUGering
}

\begin{abstract}
We propose a model for a two-dimensional elastic body with a thin elastic inclusion modeled by a beam equation. Moreover, we assume that a delamination of the inclusion may take place resulting in a crack. Nonlinear boundary conditions are imposed at the crack faces to prevent mutual penetration between the faces. Both variational and differential problem formulations are considered, and existence of solutions is established. Furthermore, we study the dependence of the solution on the rigidity of the embedded beam. It is proved that in the limit cases corresponding to infinite and zero rigidity, we obtain a rigid beam inclusion and cracks with nonpenetration conditions, respectively. Anisotropic behavior of the beam is also analyzed.
\end{abstract}

\section{Introduction}

The enforcement of elastic bodies using thin inclusions is a field of broad interest in solid and structural mechanics. The interplay between elastic fibers and matrix materials in general is important also in biological and medical problems involving tissues, muscles, tendon-couplings, etc. There are a number of different approaches in modeling such composites. The most classical approach assumes inextensible fibers; see, for example, [Saccomandi and Beatty 2002]. In this context, the modeling is often based directly on a finite elements. Another approach is based on a modeling of the matrix material as a supporting layer, like a Winkler support; see, for example, [Nassar and Hassen 1987]. Here, the fiber is represented by an Euler-Bernoulli beam. A very natural approach is based on asymptotic analysis [Argatov and Nazarov 1999]. Here, the embedded beams are taken with a small thickness parameter and the elastic layer is infinite. The limiting problem relates to a Winkler or Pasternak-type model. Finally, there are attempts to model hybrid partial differential equations coupling, say, the two-dimensional wave equation to a one-dimensional wave equation, using proper transmission conditions; see [Koch and Zuazua 2006].

MSC2010: 74-XX.

Keywords: thin inclusion, nonlinear boundary conditions, nonpenetration, crack, variational inequality. 
In general, the terminology "thin inclusion" is used in cases where the dimension of the inclusion is less than that of the body. Among thin inclusions we can distinguish between rigid and elastic ones. Moreover, thin inclusions have a tendency to delaminate from the matrix material, thereby introducing cracks. A mathematical theory should be capable of consistently handling these different aspects. Therefore, in order to analyze composite materials one has to consider mathematical models of deformable bodies with elastic and rigid inclusions and cracks. In such a case, new types of boundary value problems and boundary conditions appear. Cracks also can be viewed as thin inclusions of zero rigidity. There are different approaches to modeling cracks in solids. The classical models are characterized by linear boundary conditions at the crack faces [Kozlov and Maz'ya 1991; Grisvard 1992; Nazarov and Plamenevsky 1994]. These linear models allow the opposite crack faces to penetrate each other which demonstrates a shortcoming of the model from a mechanical standpoint. For a discussion of singularities at the crack tip see, for example, [Kozlov and Maz'ya 1991; Nazarov and Plamenevsky 1994]. In recent years, a crack theory with nonpenetration conditions at the crack faces has been under active study. This theory is characterized by inequality-type boundary conditions which leads to free boundary value problems. The book [Khludnev and Kovtunenko 2000] contains results on crack models with the nonpenetration conditions for a wide class of constitutive laws. Elastic behavior of bodies with cracks and inequality-type boundary conditions is analyzed in [Khludnev 2010a]. In particular, the differentiability of energy functionals with respect to crack length is investigated. Finding the derivatives of the energy functionals is important from the standpoint of the Griffith rupture criterion; see [Kovtunenko 2003; Rudoy 2007; Frémiot et al. 2009; Khludnev et al. 2010]. The asymptotic behavior of the solution near crack tips was analyzed in [Khludnev and Kozlov 2008]. Existence theorems and qualitative properties of solutions in equilibrium problems for elastic bodies with thin and volume rigid inclusions can be found in [Khludnev et al. 2009; 2010b; Neustroeva 2009; Khludnev and Leugering 2010; 2011; Rudoy 2011; Rotanova 2011]. For behavior near rigid inclusion tips, see [Itou et al. 2012].

We propose a new model of a thin elastic inclusion inside of an elastic body. We consider a planar elastic body $\Omega$ with embedded elastic fibers $\gamma_{i}, i=1, \ldots n$, as shown in the figure below. However, in this article we do not focus on the

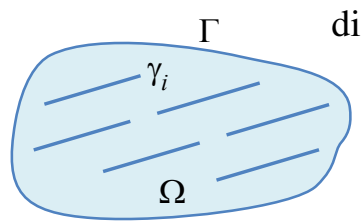
distribution of such fibers in such a domain but rather on the mathematical modeling and analysis of immersed fibers to begin with. We, therefore, without loss of generality, concentrate on a single fiber $\gamma$ embedded into $\Omega$ with boundary $\Gamma$.

The mechanical behavior of the inclusion is modeled by the Kirchhoff-Love equations. The inclusion may be delaminated, providing therefore the presence 
of a crack. To exclude a mutual penetration between the crack faces, nonlinear boundary conditions of inequality type are considered along the cracks. Different problem formulations are proposed which are shown to be equivalent to each other. We prove the existence and uniqueness of solutions and analyze limit cases describing the passage to infinity and zero of the rigidity parameter of the inclusion. In particular, the models of rigid beam inclusions, semirigid beam inclusions, and crack models with the nonpenetration conditions are obtained in the limits.

The paper is organized as follows. In Section 2, we provide the problem formulation and handle the case where no delamination takes place. In Section 3, we derive the model for a one-sided delamination along the fiber. In Sections 4 and 5 we study the limiting model, as the rigidity of the fiber tends to infinity and zero, respectively. Sections 6 and 7 are concerned with two-sided delamination along the fiber and fibers that exhibit different stiffness properties with respect to longitudinal and vertical displacements. Oblique and kinking fibers as well as branching fibers can also be handled. Moreover, other beam models can be considered. However, this is subject to a forthcoming publication.

\section{Problem formulation: the case without delamination}

Denote by $\Omega \subset \mathbb{R}^{2}$ a bounded domain with Lipschitz boundary $\Gamma$ such that $\bar{\gamma} \subset \Omega$, $\gamma=(0,1) \times\{0\}$. Denote by $v=(0,1)$ a unit normal vector to $\gamma, \tau=(1,0)$, and set $\Omega_{\gamma}=\Omega \backslash \bar{\gamma}$; see figure.

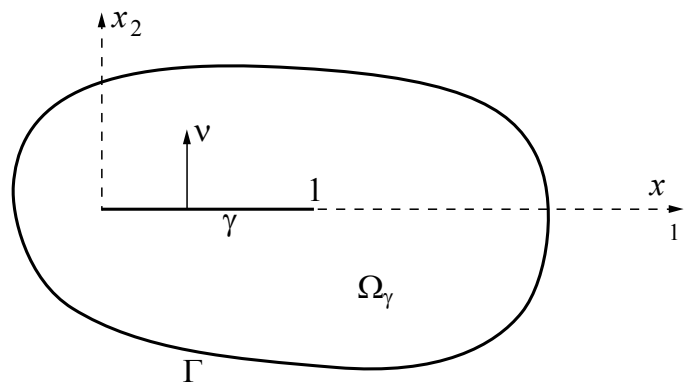

In what follows, the domain $\Omega_{\gamma}$ represents a region with an elastic material, and $\gamma$ is an elastic inclusion with specified properties. In particular, we consider $\gamma$ as a Kirchhoff-Love or Euler-Bernoulli beam incorporated in the elastic body. Let $A=\left\{a_{i j k l}\right\}, i, j, k, l=1,2$, be a given elasticity tensor with the usual properties of symmetry and positive definiteness,

$$
\begin{gathered}
a_{i j k l}=a_{j i k l}=a_{k l i j}, \quad i, j, k, l=1,2, \quad a_{i j k l} \in L^{\infty}(\Omega), \\
a_{i j k l} \xi_{i j} \xi_{k l} \geq c_{0}|\xi|^{2}, \quad \forall \xi_{j i}=\xi_{i j}, \quad c_{0}=\text { const. }>0 .
\end{gathered}
$$

A summation convention over repeated indices is used: all functions with two lower indices are assumed to be symmetric in those indices. 
An equilibrium problem for the body $\Omega_{\gamma}$ and the elastic inclusion $\gamma$ (see, for example, [Bessoud et al. 2008]) is formulated as follows. For given external forces $f=\left(f_{1}, f_{2}\right) \in L^{2}(\Omega)^{2}$ acting on the body, we want to find a displacement field $u=\left(u_{1}, u_{2}\right)$, a stress tensor $\sigma=\left\{\sigma_{i j}\right\}, i, j=1,2$, and thin inclusion displacements $v, w$, defined in $\Omega, \Omega_{\gamma}$, and $\gamma$, respectively, such that

$$
\begin{aligned}
& -\operatorname{div} \sigma=f \quad \text { in } \Omega_{\gamma}, \\
& \sigma-A \varepsilon(u)=0 \quad \text { in } \Omega \text {, } \\
& E I v_{x x x x}=\left[\sigma_{v}\right] \text { on } \gamma \text {, } \\
& -E S w_{x x}=\left[\sigma_{\tau}\right] \text { on } \gamma \text {, } \\
& u=0 \quad \text { on } \Gamma \text {, } \\
& E I v_{x x}=E I v_{x x x}=E S w_{x}=0 \quad \text { for } x=0,1 \text {, } \\
& v=u_{v}, \quad w=u_{\tau} \quad \text { on } \gamma .
\end{aligned}
$$

Here $[h]=h^{+}-h^{-}$is a jump of a function $h$ on $\gamma$, where $h^{ \pm}$are the traces of $h$ on the faces of the beam $\gamma^{ \pm}$. The signs \pm correspond to the positive and negative directions of $v ; v_{x}=d v / d x, x=x_{1},\left(x_{1}, x_{2}\right) \in \Omega ; \varepsilon(u)=\left\{\varepsilon_{i j}(u)\right\}$ is the strain tensor, $\varepsilon_{i j}(u)=\frac{1}{2}\left(u_{i, j}+u_{j, i}\right), i, j=1,2$; and $\sigma v=\left(\sigma_{1 j} v_{j}, \sigma_{2 j} v_{j}\right), \sigma_{v}=\sigma_{i j} v_{j} v_{i}$, $\sigma_{\tau}=\sigma v \cdot \tau, u_{v}=u v, u_{\tau}=u \tau$. By $E, I$, and $S$ we denote the Young's modulus, the inertia of the cross section, and the area of cross section, respectively. Below, for the sake of simplicity, we put $E I=1$ and $E S=1$. The essence of the mathematical results obtained in this article does not change by this particular choice. When it comes to the asymptotic analysis for the stiffness of the beam, the role of the stiffness parameters will be taken into account. See Sections 4, 5, and 7.

Functions defined on $\gamma$ we identify with functions of the variable $x$.

Relations (2-1), (2-3), and (2-4) are the equilibrium equations for the elastic body and the inclusion, and (2-2) represents Hooke's law. According to (2-7), the vertical and tangential (along the axis $x_{1}$ ) displacements of the elastic body coincide with the inclusion displacements at $\gamma$.

Below we provide a variational formulation of the problem (2-1)-(2-7). To this end, we introduce the Sobolev space

$$
V=\left\{(u, v, w) \in\left(H_{0}^{1}(\Omega)\right)^{2} \times H^{2}(\gamma) \times H^{1}(\gamma) \mid v=u_{v}, w=u_{\tau} \text { on } \gamma\right\},
$$

and the energy functional

$$
\Pi(u, v, w)=\frac{1}{2} \int_{\Omega} \sigma(u) \varepsilon(u)-\int_{\Omega} f u+\frac{1}{2} \int_{\gamma} v_{x x}^{2}+\frac{1}{2} \int_{\gamma} w_{x}^{2} .
$$

Here $\sigma(u)=\sigma$ is defined by (2-2), that is, $\sigma(u)=A \varepsilon(u)$, and, for simplicity, we write $\sigma(u) \varepsilon(u)=\sigma_{i j}(u) \varepsilon_{i j}(u), f u=f_{i} u_{i}$. We use standard notation for the spaces 
$\left(H_{0}^{1}(\Omega)\right)^{2}, H^{2}(\gamma), H^{1}(\gamma)$. The functions $u, v$, and $w$ are independent, and the only relations are provided by the definition of $V$. In particular, $u_{v}$ and $u_{\tau}$ have more regularity as compared to that resulting from the inclusion $u \in\left(H_{0}^{1}(\Omega)\right)^{2}$. We also use the notation $H^{s}(X)^{2}:=\left(H^{s}(X)\right)^{2}$ for Sobolev spaces concerning functions in the plane.

Consider the minimization problem

$$
\text { Find }(u, v, w) \in V \text { such that } \Pi(u, v, w)=\inf _{V} \Pi .
$$

Theorem 2.1. Problem $(\mathrm{P})$ admits a unique solution $(u, v, w)$ satisfying

$$
\begin{aligned}
(u, v, w) & \in V, \\
\int_{\Omega} \sigma(u) \varepsilon(\bar{u})-\int_{\Omega} f \bar{u}+\int_{\gamma} v_{x x} \bar{v}_{x x}+\int_{\gamma} w_{x} \bar{w}_{x}=0, & \forall(\bar{u}, \bar{v}, \bar{w}) \in V .
\end{aligned}
$$

Moreover, if the solution is smooth, then the strong representation, (2-1)-(2-7), and the weak representation, (2-8) and (2-9), are equivalent.

Proof. In order to prove that the problem (2-8) and (2-9) admits a solution, it suffices to establish the coercivity of the functional $\Pi$ on the space $V$, since its weak lower semicontinuity is obvious. Due to Korn's inequality, we have

$$
\Pi(u, v, w) \geq c_{0}\|u\|_{1, \Omega}^{2}-c_{1}\|u\|_{1, \Omega}+\frac{1}{2} \int_{\gamma}\left(v_{x x}^{2}+w_{x}^{2}\right) \pm \beta \int_{\gamma}\left(v^{2}+w^{2}\right),
$$

with positive constants $c_{0}$ and $c_{1}$ and a parameter $\beta>0$, where $\|\cdot\|_{1, \Omega}$ is the norm in $H_{0}^{1}(\Omega)^{2}$ and $\|\cdot\|_{i, \gamma}$ is the norm in $H^{i}(\gamma), i=1,2$. We have $v=u_{v}$ and $w=u_{\tau}$ at $\gamma$, hence, for small $\beta$, due to the trace inequality

$$
\frac{c_{0}}{2}\|u\|_{1, \Omega}^{2}-\beta \int_{\gamma}\left(v^{2}+w^{2}\right) \geq 0 .
$$

Thus, from (2-10) we obtain the desired limit:

$$
\begin{array}{r}
\Pi(u, v, w) \geq \frac{c_{0}}{2}\|u\|_{1, \Omega}^{2}-c_{1}\|u\|_{1, \Omega}+\frac{1}{2} \int_{\gamma}\left(v_{x x}^{2}+w_{x}^{2}\right)+\beta \int_{\gamma}\left(v^{2}+w^{2}\right) \rightarrow+\infty, \\
\|(u, v, w)\|_{V} \rightarrow \infty,
\end{array}
$$

We now show the equivalence of (2-1)-(2-7) and (2-8) and (2-9) for smooth solutions. Let (2-1)-(2-7) be fulfilled. Take $(\bar{u}, \bar{v}, \bar{w}) \in V$ and multiply (2-1), (2-3), and (2-4) by $\bar{u}, \bar{v}$, and $\bar{w}$, respectively. Integrating over $\Omega_{\gamma}$ and $\gamma$, respectively, we get

$$
\int_{\Omega_{\gamma}}(-\operatorname{div} \sigma-f) \bar{u}+\int_{\gamma}\left(v_{x x x x} \bar{v}-w_{x x} \bar{w}\right)-\int_{\gamma}\left(\left[\sigma_{\nu}\right] \bar{v}+\left[\sigma_{\tau}\right] \bar{w}\right)=0 .
$$

Hence, by the boundary conditions (2-5) and (2-6), 


$$
\int_{\Omega_{\gamma}}(\sigma(u) \varepsilon(\bar{u})-f \bar{u})+\int_{\gamma}[\sigma v] \bar{u}+\int_{\gamma}\left(v_{x x} \bar{v}_{x x}+w_{x} \bar{w}_{x}\right)-\int_{\gamma}\left(\left[\sigma_{v}\right] \bar{v}+\left[\sigma_{\tau}\right] \bar{w}\right)
$$

We have $[\sigma \nu] \bar{u}=\left[\sigma_{v}\right] \bar{u}_{v}+\left[\sigma_{\tau}\right] \bar{u}_{\tau}$ on $\gamma$. Taking into account that $(\bar{u}, \bar{v}, \bar{w}) \in V$, from (2-11) the identity (2-9) follows. In so doing, we change the integration domain $\Omega_{\gamma}$ by $\Omega$, since $[u]=[\bar{u}]=0$ on $\gamma$. Conversely, let (2-8) and (2-9) be fulfilled. We take test functions of the form $(\bar{u}, \bar{v}, \bar{w})=(\varphi, 0,0), \varphi \in C_{0}^{\infty}\left(\Omega_{\gamma}\right)^{2}$. This gives the equilibrium equation (2-1). Next, from (2-9) it follows that

$$
\begin{array}{r}
-\int_{\gamma}\left(\left[\sigma_{\nu}\right] \bar{u}_{v}+\left[\sigma_{\tau}\right] \bar{u}_{\tau}\right)+\int_{\gamma}\left(v_{x x x x} \bar{v}-w_{x x} \bar{w}\right)+\left.w_{x} \bar{w}\right|_{0} ^{1}+\left.v_{x x} \bar{v}_{x}\right|_{0} ^{1}-\left.v_{x x x} \bar{v}\right|_{0} ^{1}=0, \\
\forall(\bar{u}, \bar{v}, \bar{w}) \in V .
\end{array}
$$

Choosing here $\bar{w}=0$ and $\bar{v}=\bar{v}_{x}=0$ at $x=0,1$, the relation follows:

$$
-\int_{\gamma}\left[\sigma_{\nu}\right] \bar{u}_{\nu}-\int_{\gamma}\left[\sigma_{\tau}\right] \bar{u}_{\tau}+\int_{\gamma}\left(v_{x x x x} \bar{v}-w_{x x} \bar{w}\right)=0 .
$$

Consequently, by the equalities $\bar{v}=\bar{u}_{\nu}$, and $\bar{w}=\bar{u}_{\tau}$ on $\gamma$, we obtain (2-3) and (2-4). In such a case, the identity (2-12) implies (2-6). Hence, the equivalence of $(2-1)-(2-7)$ and (2-8) and (2-9) is proved.

\section{Delaminated elastic inclusion}

Assume that a delamination of the elastic inclusion takes place at $\gamma^{+}$, thus we have a crack. In our model, inequality-type boundary conditions will be considered to prevent a mutual penetration between the crack faces. Displacements of the inclusion should coincide with the displacements of the elastic body at $\gamma^{-}$. The problem formulation is as follows. We have to find a displacement field $u=\left(u_{1}, u_{2}\right)$, a stress tensor $\sigma=\left\{\sigma_{i j}\right\}, i, j=1,2$, and thin inclusion displacements $v$ and $w$ defined in $\Omega_{\gamma}, \Omega_{\gamma}$, and $\gamma$, respectively, such that

$$
\begin{aligned}
-\operatorname{div} \sigma & =f & & \text { in } \Omega_{\gamma}, \\
\sigma-A \varepsilon(u) & =0 & & \text { in } \Omega_{\gamma}, \\
v_{x x x x} & =\left[\sigma_{v}\right] & & \text { on } \gamma, \\
-w_{x x} & =\left[\sigma_{\tau}\right] & & \text { on } \gamma, \\
u & =0 & & \text { on } \Gamma, \\
{\left[u_{v}\right] \geq 0, \quad v=u_{v}^{-}, \quad w=u_{\tau}^{-}, \quad \sigma_{v}^{+}\left[u_{v}\right] } & =0 & & \text { on } \gamma, \\
v_{x x}=v_{x x x}=w_{x} & =0 & & \text { for } x=0,1, \\
\sigma_{v}^{+} \leq 0, \quad \sigma_{\tau}^{+} & =0 & & \text { on } \gamma .
\end{aligned}
$$


The first inequality in (3-7) provides a mutual nonpenetration between the crack faces. The second and the third relations of (3-7) show that the inclusion displacements coincide with the vertical and tangential displacements of the elastic body at $\gamma^{-}$.

First, we provide a variational formulation of the problem (3-1)-(3-8). We introduce the set of admissible displacements

$$
K=\left\{(u, v, w) \in H_{\Gamma}^{1}\left(\Omega_{\gamma}\right)^{2} \times H^{2}(\gamma) \times H^{1}(\gamma) \mid\left[u_{\nu}\right] \geq 0, v=u_{v}^{-}, w=u_{\tau}^{-} \text {on } \gamma\right\}
$$

and the energy functional

$$
\Pi_{1}(u, v, w)=\frac{1}{2} \int_{\Omega_{\gamma}} \sigma(u) \varepsilon(u)-\int_{\Omega_{\gamma}} f u+\frac{1}{2} \int_{\gamma} v_{x x}^{2}+\frac{1}{2} \int_{\gamma} w_{x}^{2},
$$

where the Sobolev space $H_{\Gamma}^{1}\left(\Omega_{\gamma}\right)$ is defined as

$$
H_{\Gamma}^{1}\left(\Omega_{\gamma}\right)=\left\{v \in H^{1}\left(\Omega_{\gamma}\right) \mid v=0 \text { on } \Gamma\right\} .
$$

Theorem 3.1. There exists a unique solution of the problem

$$
\text { Find }(u, v, w) \in K \text { such that } \Pi_{1}(u, v, w)=\inf _{K} \Pi_{1} \text {. }
$$

This solution satisfies the variational inequality

$$
(u, v, w) \in K,
$$

$$
\begin{array}{r}
\int_{\Omega_{\gamma}} \sigma(u) \varepsilon(\bar{u}-u)-\int_{\Omega_{\gamma}} f(\bar{u}-u)+\int_{\gamma} v_{x x}\left(\bar{v}_{x x}-v_{x x}\right)+\int_{\gamma} w_{x}\left(\bar{w}_{x}-w_{x}\right) \geq 0, \\
\forall(\bar{u}, \bar{v}, \bar{w}) \in K .
\end{array}
$$

Moreover, (3-1)-(3-8) and (3-10) and (3-11) are equivalent for smooth solutions.

Proof. The coercivity of the functional $\Pi_{1}$ can be proved as that in Section 2; hence, the problem (3-10) and (3-11) indeed has a solution. As for the equivalence of the representations for smooth solutions, assume that (3-1)-(3-8) hold. Take $(\bar{u}, \bar{v}, \bar{w}) \in K$ and multiply (3-1), (3-3), and (3-4) by $\bar{u}-u, \bar{v}-v$, and $\bar{w}-w$, respectively. Integrating over $\Omega_{\gamma}$ and $\gamma$, we have

$$
\int_{\Omega_{\gamma}}(-\operatorname{div} \sigma-f)(\bar{u}-u)+\int_{\gamma}\left(v_{x x x x}-\left[\sigma_{\nu}\right]\right)(\bar{v}-v)+\int_{\gamma}\left(-w_{x x}-\left[\sigma_{\tau}\right]\right)(\bar{w}-w)=0,
$$

and hence

$$
\begin{array}{r}
\int_{\Omega_{\gamma}} \sigma(u) \varepsilon(\bar{u}-u)-\int_{\Omega_{\gamma}} f(\bar{u}-u)+\int_{\gamma}[\sigma v(\bar{u}-u)]+\int_{\gamma} v_{x x}\left(\bar{v}_{x x}-v_{x x}\right) \\
+\int_{\gamma} w_{x}\left(\bar{w}_{x}-w_{x}\right)-\int_{\gamma}\left[\sigma_{v}\right](\bar{v}-v)-\int_{\gamma}\left[\sigma_{\tau}\right](\bar{w}-w)=0 .
\end{array}
$$


To prove the variational inequality (3-11), it suffices to state in (3-12) that

$$
B \equiv \int_{\gamma}[\sigma v(\bar{u}-u)]-\int_{\gamma}\left[\sigma_{\nu}\right](\bar{v}-v)-\int_{\gamma}\left[\sigma_{\tau}\right](\bar{w}-w) \leq 0 .
$$

This can be verified by (3-7) and (3-8). Hence the variational inequality (3-11) follows from (3-12), as required.

Conversely, let (3-10) and (3-11) be fulfilled. First, it is easy to derive the equilibrium equation (3-1) from (3-10) and (3-11). We next substitute the test functions $(\bar{u}, \bar{v}, \bar{w})=(u, v, w) \pm(\varphi, \omega, \psi)$ in (3-11), with $\left[\varphi_{\nu}\right]=0, \varphi_{\nu}^{-}=\omega$, $\varphi_{\tau}^{-}=\psi$, on $\gamma$. This gives

$$
\int_{\Omega_{\gamma}} \sigma(u) \varepsilon(\varphi)-\int_{\Omega_{\gamma}} f \varphi+\int_{\gamma} v_{x x} \omega_{x x}+\int_{\gamma} w_{x} \psi_{x}=0 .
$$

Hence,

$$
-\int_{\gamma}[\sigma v \cdot \varphi]+\int_{\gamma} v_{x x x x} \omega-\int_{\gamma} w_{x x} \psi-\left.v_{x x x} \omega\right|_{0} ^{1}+\left.v_{x x} \omega_{x}\right|_{0} ^{1}+\left.w_{x} \psi\right|_{0} ^{1}=0 .
$$

Assuming $\omega=\omega_{x}=\psi=0$ as $x=0,1$, from (3-13) one gets

$$
-\int_{\gamma}\left(\left[\sigma_{\nu}\right] \varphi_{\nu}+\left[\sigma_{\tau} \varphi_{\tau}\right]\right)+\int_{\gamma}\left(v_{x x x x} \omega-w_{x x} \psi\right)=0 .
$$

Due to the arbitrariness of $\varphi_{\tau}^{+}$, we obtain $\sigma_{\tau}^{+}=0$ on $\gamma$. Since $\omega=\varphi_{\nu}$ and $\psi=\varphi_{\tau}^{-}$ on $\gamma$ we obtain the equations (3-3) and (3-4). Now, taking into account (3-3) and (3-4), it follows from (3-13) that boundary conditions (3-6) are fulfilled. Let us prove the last relation of (3-7) and the inequality in (3-8). To this end, we take in (3-11) test functions of the form $(\bar{u}, \bar{v}, \bar{w})=(u, v, w)+(\varphi, 0,0)$, with $\varphi_{v}^{+} \geq 0$ on $\gamma, \varphi_{\nu}^{-}=0$, and $\varphi_{\tau}^{-}=0$ on $\gamma$. This provides

$$
\int_{\Omega_{\gamma}} \sigma(u) \varepsilon(\varphi)-\int_{\Omega_{\gamma}} f \varphi \geq 0,
$$

and thus

$$
-\int_{\gamma} \sigma^{+} v \cdot \varphi^{+} \geq 0
$$

This relation implies

$$
\int_{\gamma} \sigma_{v}^{+} \varphi_{\nu}^{+} \leq 0
$$

Since $\varphi_{\nu}^{+}$is an arbitrary nonnegative function, we conclude that $\sigma_{\nu}^{+} \leq 0$ on $\gamma$.

Next, assume that at any point $y \in \gamma$ we have $\left[u_{v}(y)\right]>0$. It necessarily gives $\sigma_{v}^{+}(y)=0$, since in such a case a function $(\bar{u}, \bar{v}, \bar{w})=(u, v, w) \pm(\lambda \varphi, 0,0)$ can be 
substituted in (3-11) with a smooth function $\varphi, \operatorname{supp} \varphi \subset \bar{D}, \lambda$ a small parameter, and $D$ a small neighborhood:

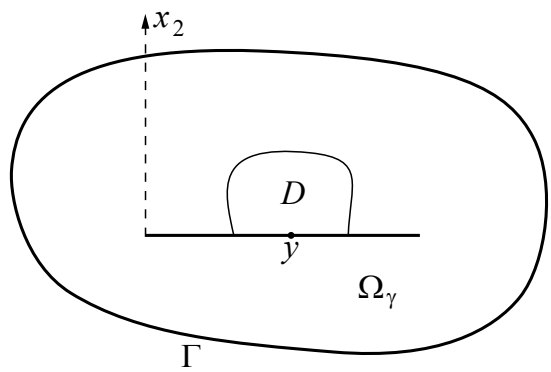

This provides the relation

$$
\int_{\Omega_{\gamma}} \sigma(u) \varepsilon(\varphi)-\int_{\Omega_{\gamma}} f \varphi=0
$$

hence the statement follows. On the other hand, if $\sigma_{v}^{+}(y)<0$ we derive $\left[u_{v}(y)\right]=0$, and, consequently, the last relation of (3-7) is proved. The proof of the equivalency of (3-1)-(3-8) and (3-10) and (3-11) is complete.

\section{Convergence as the rigidity tends to infinity}

In fact, a solution of the problem (3-1)-(3-8) should depend on the rigidity parameter of the thin inclusion. In the model (3-1)-(3-8), this parameter was taken to be equal to 1 . In this section we introduce the parameter into the model and analyze its passage to infinity. To this end, we define the energy functional

$$
\Pi_{\delta}(u, v, w)=\frac{1}{2} \int_{\Omega_{\gamma}} \sigma(u) \varepsilon(u)-\int_{\Omega_{\gamma}} f u+\frac{\delta}{2} \int_{\gamma} v_{x x}^{2}+\frac{\delta}{2} \int_{\gamma} w_{x}^{2}, \quad \delta>0 .
$$

Theorem 4.1. There exists a unique solution to the problem

$$
\text { Find }\left(u^{\delta}, v^{\delta}, w^{\delta}\right) \in K \text { such that } \Pi_{\delta}\left(u^{\delta}, v^{\delta}, w^{\delta}\right)=\inf _{K} \Pi_{\delta}
$$

that satisfies the variational inequality

$$
\left(u^{\delta}, v^{\delta}, w^{\delta}\right) \in K,
$$

$$
\begin{array}{r}
\int_{\Omega_{\gamma}} \sigma\left(u^{\delta}\right) \varepsilon\left(\bar{u}-u^{\delta}\right)-\int_{\Omega_{\gamma}} f\left(\bar{u}-u^{\delta}\right)+\delta \int_{\gamma} v_{x x}^{\delta}\left(\bar{v}_{x x}-v_{x x}^{\delta}\right)+\delta \int_{\gamma} w_{x}^{\delta}\left(\bar{w}_{x}-w_{x}^{\delta}\right) \geq 0, \\
\forall(\bar{u}, \bar{v}, \bar{w}) \in K .
\end{array}
$$

Proof. The proof is analogous to that of Theorem 2.1 and is omitted.

Our aim in this section is to pass to the limit in (4-1) and (4-2) as $\delta \rightarrow+\infty$. To this end, we introduce the notation for vertical rigid displacements $R_{S}(\gamma)$ and for 
admissible displacements $K_{r}$ :

$$
\begin{aligned}
R_{s}(\gamma) & :=\left\{l(x) \mid l(x)=c_{0}+c_{1} x, x \in \gamma ; c_{0}, c_{1} \in \mathbb{R}\right\}, \\
K_{r} & :=\left\{u \in H_{\Gamma}^{1}\left(\Omega_{\gamma}\right)^{2}\left|\left[u_{\nu}\right] \geq 0, u_{\nu}^{-}\right|_{\gamma} \in R_{s}(\gamma),\left.u_{\tau}^{-}\right|_{\gamma} \in \mathbb{R}\right\} .
\end{aligned}
$$

Theorem 4.2. Let $\left(u^{\delta}, v^{\delta}, w^{\delta}\right) \in K$. Then we can pass to the limit as $\delta \rightarrow+\infty$ and obtain a unique element $(u, v, w) \in K_{r}$ such that $(u, v, w)$ satisfies

$$
\begin{array}{llll}
u^{\delta} \rightarrow u & \text { weakly in } H_{\Gamma}^{1}\left(\Omega_{\gamma}\right)^{2}, & & \\
v^{\delta} \rightarrow v & \text { weakly in } H^{2}(\gamma), & v_{x x}=0 & \text { on } \gamma, \\
w^{\delta} \rightarrow w & \text { weakly in } H^{1}(\gamma), & w_{x}=0 & \text { on } \gamma .
\end{array}
$$

In particular, $v(x)=c_{0}+c_{1} x, w(x)=q_{0}, q_{0}=$ const., $x \in(0,1)$. Moreover, $(u, v, w)$ satisfies the limiting problem

$$
\begin{aligned}
& u \in K_{r}, \\
& \int_{\Omega_{\gamma}} \sigma(u) \varepsilon(\bar{u}-u)-\int_{\Omega_{\gamma}} f(\bar{u}-u) \geq 0, \quad \forall \bar{u} \in K_{r} .
\end{aligned}
$$

Proof. From (4-2) it follows that

$$
\int_{\Omega_{\gamma}} \sigma\left(u^{\delta}\right) \varepsilon\left(u^{\delta}\right)-\int_{\Omega_{\gamma}} f u^{\delta} \pm \beta \int_{\gamma}\left(\left(w^{\delta}\right)^{2}+\left(v^{\delta}\right)^{2}\right)+\delta \int_{\gamma}\left(v_{x x}^{\delta}\right)^{2}+\delta \int_{\gamma}\left(w_{x}^{\delta}\right)^{2}
$$

For small $\beta>0$, due to $v^{\delta}=u_{v}^{\delta-}$ and $w^{\delta}=u_{\tau}^{\delta-}$ on $\gamma$, the following relation holds:

$$
\frac{1}{2} \int_{\Omega_{\gamma}} \sigma\left(u^{\delta}\right) \varepsilon\left(u^{\delta}\right)-\beta \int_{\gamma}\left(\left(w^{\delta}\right)^{2}+\left(v^{\delta}\right)^{2}\right) \geq 0 .
$$

Consequently, from (4-8) one gets, as $\delta \geq \beta$,

$$
c_{0}\left\|u^{\delta}\right\|_{1, \Omega_{\gamma}}^{2}+\beta\left\|v^{\delta}\right\|_{2, \gamma}^{2}+\beta\left\|w^{\delta}\right\|_{1, \gamma}^{2} \leq c_{1}\left\|u^{\delta}\right\|_{1, \Omega_{\gamma}}, \quad c_{0}>0 .
$$

Hence, uniformly in $\delta \geq \delta_{0}$,

$$
\left\|u^{\delta}\right\|_{1, \Omega_{\gamma}}^{2}+\left\|v^{\delta}\right\|_{2, \gamma}^{2}+\left\|w^{\delta}\right\|_{1, \gamma}^{2} \leq c .
$$

On the other hand, the relation (4-8) implies for $\delta \geq \delta_{0}$,

$$
\delta \int_{\gamma}\left(v_{x x}^{\delta}\right)^{2}+\delta \int_{\gamma}\left(w_{x}^{\delta}\right)^{2} \leq c .
$$

Thus, we can pass to the limit on a subsequence and obtain (4-3)-(4-5). Let us choose $(\bar{u}, l, q) \in K$ as a test function in (4-2), $l \in R_{s}(\gamma), q \in \mathbb{R}$. Notice that 
$\bar{u} \in K_{r}$. Then, from (4-2) it follows that

$$
\int_{\Omega_{\gamma}} \sigma\left(u^{\delta}\right) \varepsilon\left(\bar{u}-u^{\delta}\right)-\int_{\Omega_{\gamma}} f\left(\bar{u}-u^{\delta}\right) \geq \delta \int_{\gamma}\left(v_{x x}^{\delta}\right)^{2}+\delta \int_{\gamma}\left(w_{x}^{\delta}\right)^{2} .
$$

Again, passing to the limit, as $\delta \rightarrow \infty$, according to (4-3)-(4-5) we obtain the variational inequality (4-6) and (4-7) just as in [Khludnev 2010a; 2010b; Khludnev and Leugering 2010], with $\left.u\right|_{\gamma}=\rho$, where, for any $x \in \gamma$, we have

$$
\rho(x)=b\left(x_{2},-x_{1}\right)+\left(a_{1}, a_{2}\right), \quad \text { with } a_{1}, a_{2}, b \in \mathbb{R} .
$$

Hence $u$ is an infinitesimal rigid displacement at $\gamma$. The convergence of the entire sequence and the uniqueness follows as usual.

Remark. The inclusion $\gamma$ in the limit problem (4-6) and (4-7) can be interpreted as a rigid beam inclusion. Solvability of this problem can be also proved independently by minimizing the functional

$$
\pi(v)=\frac{1}{2} \int_{\Omega_{\gamma}} \sigma(v) \varepsilon(v)-\int_{\Omega_{\gamma}} f v
$$

over the set $K_{r}$.

We are now going to establish two strong formulations of (4-6) and (4-7), which, in turn, are equivalent to (4-6) and (4-7) if the solutions are smooth.

Theorem 4.3. We consider two problems:

(i) Find a displacement field $u=\left(u_{1}, u_{2}\right)$, a stress tensor $\sigma=\left\{\sigma_{i j}\right\}, i, j=1,2$, and thin inclusion displacements $l_{0} \in R_{s}(\gamma)$, and $q_{0} \in \mathbb{R}$ defined in $\Omega_{\gamma}, \Omega_{\gamma}$, and $\gamma$, respectively, such that

$$
\begin{aligned}
-\operatorname{div} \sigma & =f & & \text { in } \Omega_{\gamma}, \\
\sigma-A \varepsilon(u) & =0 & & \text { in } \Omega_{\gamma}, \\
u & =0 & & \text { on } \Gamma, \\
{\left[u_{v}\right] \geq 0, \quad l_{0}=u_{v}^{-}, \quad q_{0} } & =u_{\tau}^{-} & & \text {on } \gamma, \\
\int_{\gamma}[\sigma v \cdot u] & =0, & & \\
-\int_{\gamma}[\sigma v \cdot \bar{u}] & \geq 0, & & \forall \bar{u} \in K_{r} .
\end{aligned}
$$

(ii) Find a displacement field $u=\left(u_{1}, u_{2}\right)$, a stress tensor $\sigma=\left\{\sigma_{i j}\right\}, i, j=1,2$, and thin inclusion displacements $l_{0} \in R_{s}(\gamma)$ and $q_{0} \in \mathbb{R}$ defined in $\Omega_{\gamma}, \Omega_{\gamma}$, 
and $\gamma$, respectively, such that

$$
\begin{aligned}
-\operatorname{div} \sigma & =f & & \text { in } \Omega_{\gamma}, \\
\sigma-A \varepsilon(u) & =0 & & \text { in } \Omega_{\gamma}, \\
u & =0 & & \text { on } \Gamma, \\
{\left[u_{v}\right] \geq 0, \quad l_{0}=u_{v}^{-}, \quad q_{0} } & =u_{\tau}^{-} & & \text {on } \gamma, \\
\sigma_{\tau}^{+}=0, \quad \sigma_{v}^{+} \leq 0, \quad \sigma_{v}^{+}\left[u_{v}\right] & =0 & & \text { on } \gamma, \\
\int_{\gamma} \sigma_{\tau}^{-}=0, \quad \int_{\gamma}\left[\sigma_{v}\right] l & =0, & & \forall l \in R_{s}(\gamma) .
\end{aligned}
$$

(The conditions in (4-23) guarantee that the principal vector of forces and the principal vector of moments acting at $\gamma$ are equal to zero.)

Then, if the solution to problem (4-6) and (4-7) of Theorem 4.1 is smooth enough, the two problems are equivalent.

Proof. We first prove that (4-6) and (4-7) and (4-12)-(4-17) are equivalent for smooth solutions. Assume that (4-6) and (4-7) hold. We take test functions $\bar{u}$ in (4-7) such that $\bar{u}=u \pm \varphi, \varphi \in C_{0}^{\infty}\left(\Omega_{\gamma}\right)^{2}$. This provides the equilibrium equation (4-12). From (4-7) it follows

$$
\int_{\Omega_{\gamma}} \sigma(u) \varepsilon(u)-\int_{\Omega_{\gamma}} f u=0 .
$$

Integrating by parts in (4-24) we get (4-16). By (4-24), the variational inequality (4-7) can be rewritten as

$$
\int_{\Omega_{\gamma}} \sigma(u) \varepsilon(\bar{u})-\int_{\Omega_{\gamma}} f \bar{u} \geq 0, \quad \forall \bar{u} \in K_{r},
$$

thus (4-17) follows. Conversely, let (4-12)-(4-17) be fulfilled. We take $\bar{u} \in K_{r}$ and multiply (4-12) by $\bar{u}-u$. Integrating over $\Omega_{\gamma}$ we get

$$
\int_{\Omega_{\gamma}}(-\operatorname{div} \sigma-f)(\bar{u}-u)=0 .
$$

Hence

$$
\int_{\gamma}[\sigma v(\bar{u}-u)]+\int_{\Omega_{\gamma}} \sigma(u) \varepsilon(\bar{u}-u)-\int_{\Omega_{\gamma}} f(\bar{u}-u)=0 .
$$

In order to obtain the variational inequality (4-7), it suffices to prove

$$
-\int_{\gamma}[\sigma v(\bar{u}-u)] \geq 0 .
$$

But the inequality (4-25) follows from (4-16) and (4-17). Thus, the equivalence of (4-6) and (4-7) and (4-12)-(4-17) is established. 
We now turn to the second problem and demonstrate that (4-6)-(4-7) is equivalent to (4-18)-(4-23) for smooth solutions. Let (4-6) and (4-7) be fulfilled. As before, we check that the equilibrium equation (4-18) follows from (4-7). Next, we choose test functions $\bar{u}=u \pm \tilde{u},\left[\tilde{u}_{v}\right]=0,\left.\tilde{u}_{v}^{-}\right|_{\gamma} \in R_{s}(\gamma),\left.\tilde{u}_{\tau}^{-}\right|_{\gamma} \in \mathbb{R}$, and $\tilde{u} \in H_{\Gamma}^{1}\left(\Omega_{\gamma}\right)^{2}$. This gives

$$
\int_{\Omega_{\gamma}} \sigma(u) \varepsilon(\tilde{u})-\int_{\Omega_{\gamma}} f \tilde{u}=0
$$

and, hence,

$$
-\int_{\gamma}\left[\sigma_{\nu}\right] \tilde{u}_{\nu}-\int_{\gamma}\left[\sigma_{\tau} \tilde{u}_{\tau}\right]=0
$$

Since $\tilde{u}_{\tau}^{+}$is arbitrary on $\gamma$, we derive the first relation of (4-22). By $\tilde{u}_{\tau}^{-} \in \mathbb{R}$ on $\gamma$, from (4-26) we also obtain the first and the second relations of (4-23). Now we choose test functions in (4-7) as $\bar{u}=u+\tilde{u}, \tilde{u} \in H_{\Gamma}^{1}\left(\Omega_{\gamma}\right)^{2}$, and $\tilde{u}_{v}^{+} \geq 0$ on $\gamma$, supp $\tilde{u} \subset \bar{D}$; see figure on page 9 . This gives

$$
\int_{\Omega_{\gamma}} \sigma(u) \varepsilon(\tilde{u})-\int_{\Omega_{\gamma}} f \tilde{u} \geq 0 .
$$

Consequently,

$$
\int_{\gamma}\left(\sigma_{v}^{+} \tilde{u}_{v}^{+}+\sigma_{\tau}^{+} \tilde{u}_{\tau}^{+}\right) \leq 0
$$

By the choice of $\tilde{u}$, from (4-27) the second relation of (4-22) follows.

In order to derive (4-18)-(4-23) from (4-6) and (4-7), it remains to check the last condition of (4-22). To this end, assume that at a given point $y \in \gamma$ we have $\left[u_{v}(y)\right]>0$. Take test functions in (4-7) of the form $\bar{u}=u \pm \lambda \varphi$, supp $\varphi \subset \bar{D}$, where $\lambda$ is a small parameter, $D$ is a small neighborhood, and $\varphi$ is a smooth function; see again figure on page 9 . We get

$$
\int_{\Omega_{\gamma}} \sigma(u) \varepsilon(\varphi)-\int_{\Omega_{\gamma}} f \varphi=0
$$

thus

$$
\int_{\gamma} \sigma_{v}^{+} \varphi_{\nu}^{+}=0
$$

and $\sigma_{v}^{+}(y)=0$, that is, $\sigma_{v}^{+}(y)\left[u_{v}(y)\right]=0$. On the other hand, assuming that $\sigma_{v}^{+}(y)<0$, we easily derive $\left[u_{v}(y)\right]=0$, and the last relation of (4-22) follows. Thus, from (4-6) and (4-7) we have derived all relations (4-18)-(4-23). To complete the proof of equivalence of (4-6) and (4-7) and (4-18)-(4-23), assume the converse, that is, let (4-18)-(4-23) be fulfilled. We take $\bar{u} \in K_{r}$ and multiply (4-18) by $\bar{u}-u$. 
Integrating over $\Omega_{\gamma}$, one gets

$$
\int_{\Omega_{\gamma}}(-\operatorname{div} \sigma-f)(\bar{u}-u)=0
$$

and, consequently,

$$
\int_{\Omega_{\gamma}} \sigma(u) \varepsilon(\bar{u}-u)-\int_{\Omega_{\gamma}} f(\bar{u}-u)=-\int_{\gamma}[\sigma v(\bar{u}-u)] .
$$

To derive the variational inequality (4-7) from (4-28), it suffices to prove

$$
-\int_{\gamma}[\sigma v(\bar{u}-u)] \geq 0 .
$$

We have, by (4-22) and by $\bar{u} \in K_{r}$, that

$$
-\int_{\gamma} \sigma_{v}^{+}\left(\left[\bar{u}_{v}\right]-\left[u_{v}\right]\right) \geq 0 .
$$

In view of (4-23) and the first relation of (4-22), the inequality (4-30) can be rewritten as

$$
-\int_{\gamma}\left[\sigma_{\nu}\left(\bar{u}_{v}-u_{v}\right)\right]-\int_{\gamma}\left[\sigma_{\tau}\left(\bar{u}_{\tau}-u_{\tau}\right)\right] \geq 0 .
$$

From (4-31), (4-29) follows. We already mentioned that from (4-28) and (4-29) the variational inequality (4-7) follows. Thus, equivalence of (4-6) and (4-7) and (4-18)-(4-23) is completely proved.

\section{Convergence as the rigidity tends to zero}

In this section we analyze the case where the rigidity parameter $\delta$ for the inclusion convergence to zero. Again, consider the problem (4-1) and (4-2). Our aim is to pass to the limit in (4-1) and (4-2) as $\delta \rightarrow 0$. To this end, we define the set of admissible displacements

$$
K_{0}=\left\{u \in H_{\Gamma}^{1}\left(\Omega_{\gamma}\right)^{2} \mid\left[u_{\nu}\right] \geq 0 \text { on } \gamma\right\} .
$$

Theorem 5.1. Let $\left(u^{\delta}, v^{\delta}, w^{\delta}\right) \in K$ be the unique solution of (4-1) and (4-2). Then, as $\delta \rightarrow 0$, we find a unique element $w \in K_{0}$ such that

$$
\begin{aligned}
u^{\delta} \rightarrow u & \text { weakly in } H_{\Gamma}^{1}\left(\Omega_{\gamma}\right)^{2}, \\
\sqrt{\delta} v^{\delta} \rightarrow \tilde{v} & \text { weakly in } H^{2}(\gamma), \\
\sqrt{\delta} w^{\delta} \rightarrow \tilde{w} & \text { weakly in } H^{1}(\gamma) .
\end{aligned}
$$


Moreover, $(u, v, w)$ satisfies the variational inequality

$$
\begin{aligned}
u & \in K_{0}, \\
\int_{\Omega_{\gamma}} \sigma(u) \varepsilon(\bar{u}-u)-\int_{\Omega_{\gamma}} f(\bar{u}-u) \geq 0, \quad \forall \bar{u} & \in K_{0} .
\end{aligned}
$$

Proof. First note that (4-2) implies

$$
\int_{\Omega_{\gamma}} \sigma\left(u^{\delta}\right) \varepsilon\left(u^{\delta}\right)-\int_{\Omega_{\gamma}} f u^{\delta}+\delta \int_{\gamma}\left(v_{x x}^{\delta}\right)^{2}+\delta \int_{\gamma}\left(w_{x}^{\delta}\right)^{2}=0 .
$$

Hence, we have a uniform-in- $\delta$ estimate

$$
\left\|u^{\delta}\right\|_{1, \Omega_{\gamma}}^{2} \leq c
$$

On the other hand, the relation (5-6) implies, for all $\delta$,

$$
\delta \int_{\gamma}\left(v_{x x}^{\delta}\right)^{2}+\delta \int_{\gamma}\left(w_{x}^{\delta}\right)^{2} \leq c .
$$

By (5-7),

$$
\int_{\gamma}\left(v^{\delta}\right)^{2}=\int_{\gamma}\left(u_{\nu}^{\delta-}\right)^{2} \leq c, \quad \int_{\gamma}\left(w^{\delta}\right)^{2}=\int_{\gamma}\left(u_{\tau}^{\delta-}\right)^{2} \leq c,
$$

hence, in view of (5-8),

$$
\delta\left\|v^{\delta}\right\|_{2, \gamma}^{2}+\delta\left\|w^{\delta}\right\|_{1, \gamma}^{2} \leq c .
$$

By (5-1)-(5-3), a passage to the limit in (4-1) and (4-2) is possible. We choose $\bar{u} \in K_{0}$ such that $\bar{u}_{v}$ and $\bar{u}_{\tau}$ are smooth at $\gamma^{-}$, and define the functions $\bar{v}=\bar{u}_{v}^{-}$and $\bar{w}=\bar{u}_{\tau}^{-}$on $\gamma$. Then $(\bar{u}, \bar{v}, \bar{w}) \in K$, and a substitution of this test function in (4-2) implies

$$
\begin{aligned}
\int_{\Omega_{\gamma}} \sigma\left(u^{\delta}\right) \varepsilon(\bar{u})- & \int_{\Omega_{\gamma}} f\left(\bar{u}-u^{\delta}\right) \\
& \geq \int_{\Omega_{\gamma}} \sigma\left(u^{\delta}\right) \varepsilon\left(u^{\delta}\right)+\delta \int_{\gamma}\left(v_{x x}^{\delta}\right)^{2}-\delta \int_{\gamma} v_{x x}^{\delta} \bar{v}_{x x}+\delta \int_{\gamma}\left(w_{x}^{\delta}\right)^{2}-\delta \int_{\gamma} w_{x}^{\delta} \bar{w}_{x} .
\end{aligned}
$$

Taking the lower limit as $\delta \rightarrow 0$ in both parts of this inequality, we derive

$$
\int_{\Omega_{\gamma}} \sigma(u) \varepsilon(\bar{u}-u)-\int_{\Omega_{\gamma}} f(\bar{u}-u) \geq 0 .
$$

Inequality (5-10) holds for all functions $\bar{u} \in K_{0}$ such that $\bar{u}_{v}$ and $\bar{u}_{\tau}$ are quite smooth at $\gamma^{-}$. We state that it will be valid for all $\bar{u} \in K_{0}$. Indeed, let $\bar{u} \in K_{0}$ be any fixed 
function. We divide the domain $\Omega_{\gamma}$ into two subdomains $\Omega_{1}$ and $\Omega_{2}$, as shown:

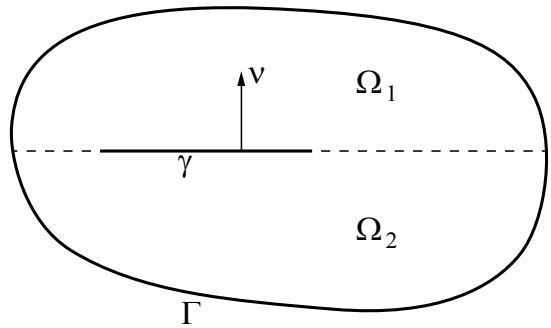

Consider the restriction $\left.\bar{u}\right|_{\Omega_{2}} \in H^{1}\left(\Omega_{2}\right)^{2}$, and extend this function to $\Omega$ as a function from $H_{0}^{1}(\Omega)^{2}$. Denote this extension by $v$. Then we put $\tilde{u}=\bar{u}-v$. It is clear that $\left[\tilde{u}_{v}\right] \geq 0$ on $\gamma$, and $\tilde{u}=0$ in $\Omega_{2}$, thus $\tilde{u}_{v}=0$ and $\tilde{u}_{\tau}=0$ at $\gamma^{-}$. Next we choose a sequence $v^{n} \in C_{0}^{\infty}(\Omega)^{2}$ such that

$$
v^{n} \rightarrow v \quad \text { strongly in } H_{0}^{1}(\Omega)^{2} .
$$

In this case

$$
\tilde{u}+v^{n} \rightarrow \bar{u} \quad \text { strongly in } H_{\Gamma}^{1}\left(\Omega_{\gamma}\right)^{2} .
$$

On the other hand, $\tilde{u}+v^{n} \in K_{0}$, and $\tilde{u}_{v}+v_{v}^{n}$ and $\tilde{u}_{\tau}+v_{\tau}^{n}$ are smooth functions at $\gamma^{-}$. Hence, the limit function $u$ from (5-1) satisfies the variational inequality (5-4), as stated.

Remark. We have proved that the limit problem for (4-1) and (4-2) as $\delta \rightarrow 0$ coincides with the well-known boundary value problem describing the equilibrium of the elastic body with the crack $\gamma$. This model provides a mutual nonpenetration between the crack faces, hence it is suitable from the mechanical standpoint. The strong formulation of the problem (5-4) and (5-5) is as follows. We have to find functions $u=\left(u_{1}, u_{2}\right)$ and $\sigma=\left\{\sigma_{i j}\right\}, i, j=1,2$, defined in $\Omega_{\gamma}$, such that

$$
\begin{aligned}
-\operatorname{div} \sigma=f & \text { in } \Omega_{\gamma}, \\
\sigma-A \varepsilon(u)=0 & \text { in } \Omega_{\gamma}, \\
u=0 & \text { on } \Gamma, \\
{\left[u_{v}\right] \geq 0, \quad \sigma_{v}^{ \pm} \leq 0, \quad\left[\sigma_{v}\right]=0, \quad \sigma_{\tau}^{ \pm}=0, \quad \sigma_{\nu}\left[u_{v}\right]=0 } & \text { on } \gamma .
\end{aligned}
$$

Many results concerning this model can be found in [Khludnev and Kovtunenko 2000; Khludnev 2010a].

\section{Two-sided delamination of the inclusion}

In this section we analyze the case when a delamination takes place at both sides of the elastic inclusion $\gamma$. First, we remark that a delamination of the elastic inclusion can be considered at $\gamma_{0}^{+}$, where $\gamma_{0}$ is a part of $\gamma$. In particular, set $\gamma_{0}=\left(0, \frac{1}{2}\right) \times\{0\}$. 
Suppose that there is no delamination at $\gamma \backslash \gamma_{0}$. In this case a differential formulation of the equilibrium problem is as follows.

Theorem 6.1. We consider the following problem: Find a displacement field $u=$ $\left(u_{1}, u_{2}\right)$, a stress tensor $\sigma=\left\{\sigma_{i j}\right\}, i, j=1,2$, and thin inclusion displacements $v$ and $w$ defined in $\Omega_{\gamma}, \Omega_{\gamma}$, and $\gamma$, respectively, such that

$$
\begin{aligned}
& -\operatorname{div} \sigma=f \quad \text { in } \Omega_{\gamma}, \\
& \sigma-A \varepsilon(u)=0 \quad \text { in } \Omega_{\gamma} \text {, } \\
& v_{x x x x}=\left[\sigma_{v}\right] \text { on } \gamma \text {, } \\
& -w_{x x}=\left[\sigma_{\tau}\right] \text { on } \gamma \text {, } \\
& u=0 \quad \text { on } \Gamma \text {, } \\
& v_{x x}=v_{x x x}=0, \quad w_{x}=0 \quad \text { for } x=0,1, \\
& v=u_{\nu}, \quad w=u_{\tau} \quad \text { on } \gamma \backslash \gamma_{0}, \\
& {\left[u_{v}\right] \geq 0, \quad v=u_{v}^{-}, \quad w=u_{\tau}^{-} \quad \text { on } \gamma_{0},} \\
& \sigma_{v}^{+} \leq 0, \quad \sigma_{\tau}^{+}=0, \quad \sigma_{v}^{+}\left[u_{v}\right]=0 \quad \text { on } \gamma_{0} \text {. }
\end{aligned}
$$

The problem (6-1)-(6-9) admits a variational formulation.

Proof. The arguments are similar to those of the proofs above. The details are omitted.

Moreover, we can consider a different type of delamination along $\gamma$. Denote $\gamma_{1}=\left(0, \frac{2}{3}\right) \times\{0\}$ and $\gamma_{2}=\left(\frac{1}{3}, 1\right) \times\{0\}$, and assume that delamination takes place at $\gamma_{1}^{+}$and $\gamma_{2}^{-}$. In this case the part $\left(\frac{1}{3}, \frac{2}{3}\right) \times\{0\}$ of the inclusion is delaminated at both sides. We introduce the energy functional

$$
\Pi_{1}(u, v, w)=\frac{1}{2} \int_{\Omega_{\gamma}} \sigma(u) \varepsilon(u)-\int_{\Omega_{\gamma}} f u+\frac{1}{2} \int_{\gamma} v_{x x}^{2}+\frac{1}{2} \int_{\gamma} w_{x}^{2},
$$

and the set of admissible displacements

$$
\begin{aligned}
K_{1}=\{ & (u, v, w) \in H_{\Gamma}^{1}\left(\Omega_{\gamma}\right)^{2} \times H^{2}(\gamma) \times H^{1}(\gamma) \mid \\
& {\left[u_{v}\right] \geq 0 \text { on } \gamma \backslash\left(\gamma_{1} \cap \gamma_{2}\right) ; v=u_{v}^{-}, w u_{\tau}^{-} \text {on } \gamma \backslash \gamma_{2} ; } \\
& \left.v=u_{v}^{+}, w=u_{\tau}^{+} \text {on } \gamma \backslash \gamma_{1} ; u_{v}^{+}-v \geq 0, v-u_{v}^{-} \geq 0 \text { on } \gamma_{1} \cap \gamma_{2}\right\} .
\end{aligned}
$$

Theorem 6.2. There exists a unique solution to the problem:

$$
\text { Find }(u, v, w) \in K_{1} \text { such that } \Pi_{1}(u, v, w)=\inf _{K_{1}} \Pi_{1} \text {. }
$$

This solution satisfies the variational inequality

$$
(u, v, w) \in K_{1},
$$




$$
\begin{array}{r}
\int_{\Omega_{\gamma}} \sigma(u) \varepsilon(\bar{u}-u)-\int_{\Omega_{\gamma}} f(\bar{u}-u)+\int_{\gamma} v_{x x}\left(\bar{v}_{x x}-v_{x x}\right)+\int_{\gamma} w_{x}\left(\bar{w}_{x}-w_{x}\right) \geq 0, \\
\forall(\bar{u}, \bar{v}, \bar{w}) \in K_{1} .
\end{array}
$$

Moreover, if $(u, v, w) \in K_{1}$ is a smooth solution of (6-10) and (6-11) then it solves the following strong problem and vice versa:

Find a displacement field $u=\left(u_{1}, u_{2}\right)$, a stress tensor $\sigma=\left\{\sigma_{i j}\right\}, i, j=1,2$, and thin inclusion displacements $v$ and $w$ defined in $\Omega_{\gamma}, \Omega_{\gamma}$, and $\gamma$, respectively, such that

$$
\begin{aligned}
& -\operatorname{div} \sigma=f \quad \text { in } \Omega_{\gamma}, \\
& \sigma-A \varepsilon(u)=0 \quad \text { in } \Omega_{\gamma} \text {, } \\
& v_{x x x x}=\left[\sigma_{\nu}\right] \text { on } \gamma \text {, } \\
& -w_{x x}=\left[\sigma_{\nu}\right] \text { on } \gamma \text {, } \\
& u=0 \quad \text { on } \Gamma \text {, } \\
& v_{x x}=v_{x x x}=0, \quad w_{x}=0 \quad \text { for } x=0,1, \\
& v=u_{v}^{-}, \quad w=u_{\tau}^{-}, \quad \sigma_{v}^{+} \leq 0, \quad \sigma_{v}^{+}\left[u_{v}\right]=0 \quad \text { on } \gamma \backslash \gamma_{2}, \\
& {\left[u_{\nu}\right] \geq 0, \quad \sigma_{\tau}^{+}=0 \quad \text { on } \gamma \backslash \gamma_{2},} \\
& v=u_{v}^{+}, \quad w=u_{\tau}^{+}, \quad \sigma_{v}^{-} \leq 0, \quad \sigma_{v}^{-}\left[u_{v}\right]=0 \quad \text { on } \gamma \backslash \gamma_{1} \text {, } \\
& {\left[u_{\nu}\right] \geq 0, \quad \sigma_{\tau}^{-}=0 \quad \text { on } \gamma \backslash \gamma_{1} \text {, }} \\
& u_{v}^{+}-v \geq 0, \quad \sigma_{\tau}^{+}=0, \quad \sigma_{v}^{+} \leq 0, \quad \sigma_{v}^{+}\left(u_{v}^{+}-v\right)=0 \quad \text { on } \gamma_{1} \cap \gamma_{2} \text {, } \\
& v-u_{v}^{-} \geq 0, \quad \sigma_{\tau}^{-}=0, \quad \sigma_{v}^{-} \leq 0, \quad \sigma_{v}^{-}\left(v-u_{v}^{-}\right)=0 \quad \text { on } \gamma_{1} \cap \gamma_{2} \text {. }
\end{aligned}
$$

Proof. We omit the proof, as it uses the same techniques as above.

\section{Anisotropic thin elastic inclusion}

For the sake of completeness, we consider a case when the rigidity parameters of the elastic inclusion are different in the $x_{1}$ and $x_{2}$ directions. In this section we consider passages to limits for this situation. Assume that the rigidity parameter along the axis $x_{2}$ is fixed, and we change the rigidity parameter along the axis $x_{1}$. For a given parameter $\delta>0$, the problem formulation is as follows:

Find $\left(u^{\delta}, v^{\delta}, w^{\delta}\right)$ such that

$$
\left(u^{\delta}, v^{\delta}, w^{\delta}\right) \in K,
$$

$$
\begin{aligned}
\int_{\Omega_{\gamma}} \sigma\left(u^{\delta}\right) \varepsilon & \left(\bar{u}-u^{\delta}\right)-\int_{\Omega_{\gamma}} f\left(\bar{u}-u^{\delta}\right) \\
& +\int_{\gamma} v_{x x}^{\delta}\left(\bar{v}_{x x}-v_{x x}^{\delta}\right)+\delta \int_{\gamma} w_{x}^{\delta}\left(\bar{w}_{x}-w_{x}^{\delta}\right) \geq 0, \quad \forall(\bar{u}, \bar{v}, \bar{w}) \in K .
\end{aligned}
$$


Our aim is to pass to the limit in (7-1) and (7-2) as $\delta \rightarrow+\infty$ and $\delta \rightarrow 0$. We omit a justification of the limiting procedures, and just formulate the limit problems. Observe that this justification recalls those of Sections 4 and 5.

7.1. Passage to the limit as $\delta \rightarrow+\infty$. The formulation of the limiting problem is as follows. We have to find a displacement field $u=\left(u_{1}, u_{2}\right)$, a stress tensor $\sigma=\left\{\sigma_{i j}\right\}, i, j=1,2$, and thin inclusion displacements $q_{0} \in \mathbb{R}$ and $v$ defined in $\Omega_{\gamma}$, $\Omega_{\gamma}$, and $\gamma$, respectively, such that

$$
\begin{aligned}
-\operatorname{div} \sigma & =f & & \text { in } \Omega_{\gamma}, \\
\sigma-A \varepsilon(u) & =0 & & \text { in } \Omega_{\gamma}, \\
v_{x x x x} & =\left[\sigma_{v}\right] & & \text { on } \gamma, \\
u & =0 & & \text { on } \Gamma, \\
v_{x x}=v_{x x x} & =0 & & \text { for } x=0,1, \\
{\left[u_{v}\right] \geq 0, \quad v=u_{v}^{-}, \quad q_{0} } & =v_{\tau}^{-} & & \text {on } \gamma, \\
\sigma_{v}^{+} \leq 0, \quad \sigma_{v}^{+}\left[u_{v}\right]=0, \quad \sigma_{\tau}^{+} & =0 & & \text { on } \gamma, \\
\int_{\gamma} \sigma_{\tau}^{-} & =0 . & &
\end{aligned}
$$

We remark that the inclusion $\gamma$ in the limit problem (7-3)-(7-10) can be interpreted as a semirigid beam inclusion. It is possible to give a variational formulation of the problem (7-3)-(7-10).

7.2. Passage to the limit as $\delta \rightarrow 0$. In this case the formulation of the limiting problem is the following. We have to find a displacement field $u=\left(u_{1}, u_{2}\right)$, a stress tensor $\sigma=\left\{\sigma_{i j}\right\}, i, j=1,2$, and a thin inclusion displacement $v$ defined in $\Omega_{\gamma}, \Omega_{\gamma}$, and $\gamma$, respectively, such that

$$
\begin{aligned}
-\operatorname{div} \sigma & =f & & \text { in } \Omega_{\gamma}, \\
\sigma-A \varepsilon(u) & =0 & & \text { in } \Omega_{\gamma}, \\
v_{x x x x} & =\left[\sigma_{v}\right] & & \text { on } \gamma, \\
u & =0 & & \text { on } \Gamma, \\
v_{x x}=v_{x x x} & =0 & & \text { for } x=0,1, \\
{\left[u_{v}\right] \geq 0, \quad v } & =u_{v}^{-} & & \text {on } \gamma, \\
\sigma_{v}^{+} \leq 0, \quad \sigma_{\tau}^{ \pm}=0, \quad \sigma_{v}^{+}\left[u_{v}\right] & =0 & & \text { on } \gamma .
\end{aligned}
$$

Note that the thin inclusion $\gamma$ in the limit problem (7-11)-(7-17) describes only vertical displacements of the beam, and tangential displacements of the beam coincide with the tangential displacements of the elastic body at $\gamma^{-}$. We omit a variational formulation of the problem (7-11)-(7-17) since this model was analyzed in [Khludnev and Negri 2012]. 


\section{Acknowledgements}

This work was completed during a visit to Friedrich-Alexander-University ErlangenNuremberg (FAU), and the first author thanks the university for its hospitality. It was also partially supported by RFBR (10-01-00054) and the Russian Ministry of Science and Education (project N4402). The authors also gratefully acknowledge support by the DFG-CE "Engineering of Advanced Materials" at the FAU.

\section{References}

[Argatov and Nazarov 1999] I. I. Argatov and S. A. Nazarov, "Equilibrium of an elastic body pierced by horizontal thin elastic bars", J. Appl. Mech. Tech. Phys. 40:4 (1999), 763-769.

[Bessoud et al. 2008] A.-L. Bessoud, F. Krasucki, and M. Serpilli, "Plate-like and shell-like inclusions with high rigidity”, C. R. Acad. Sci. Paris Sér. I Math. 346:11-12 (2008), 697-702.

[Frémiot et al. 2009] G. Frémiot, W. Horn, A. Laurain, M. Rao, and J. Sokołowski, "On the analysis of boundary value problems in nonsmooth domains", Dissertationes Math. 462 (2009), 1-149.

[Grisvard 1992] P. Grisvard, Singularities in boundary value problems, Recherches en Mathématiques Appliquées 22, Masson, Paris, 1992.

[Itou et al. 2012] H. Itou, A. M. Khludnev, E. M. Rudoy, and A. Tani, "Asymptotic behaviour at a tip of a rigid line inclusion in linearized elasticity", Z. Angew. Math. Mech. 92:9 (2012), 716-730.

[Khludnev 2010a] A. M. Khludnev, Elasticity problems in nonsmooth domains, Fizmatlit, Moscow, 2010.

[Khludnev 2010b] А. M. Khludnev, “Задача о трещине на границе жесткого включения в упругой пластине”, Izv. Akad. Nauk. Mekh. Tverd. Tela 2010:5 (2010), 98-110. Translated as "Problem of a crack on the boundary of a rigid inclusion in an elastic plate" in Mech. Solids 45:5 (2010), 733-742.

[Khludnev and Kovtunenko 2000] A. M. Khludnev and V. A. Kovtunenko, Analysis of cracks in solids, International Series on Advances in Fracture Mechanics 6, WIT Press, Southampton, 2000.

[Khludnev and Kozlov 2008] A. M. Khludnev and V. A. Kozlov, "Asymptotics of solutions near crack tips for Poisson equation with inequality type boundary conditions", Z. Angew. Math. Phys. 59:2 (2008), 264-280.

[Khludnev and Leugering 2010] A. M. Khludnev and G. Leugering, "On elastic bodies with thin rigid inclusions and cracks", Math. Methods Appl. Sci. 33:16 (2010), 1955-1967.

[Khludnev and Leugering 2011] A. M. Khludnev and G. Leugering, "Optimal control of cracks in elastic bodies with thin rigid inclusions", Z. Angew. Math. Mech. 91:2 (2011), 125-137.

[Khludnev and Negri 2012] A. M. Khludnev and M. Negri, "Crack on the boundary of a thin elastic inclusion inside an elastic body", Z. Angew. Math. Mech. 92:5 (2012), 341-354.

[Khludnev et al. 2009] A. M. Khludnev, A. A. Novotny, J. Sokołowski, and A. Żochowski, "Shape and topology sensitivity analysis for cracks in elastic bodies on boundaries of rigid inclusions", $J$. Mech. Phys. Solids 57:10 (2009), 1718-1732.

[Khludnev et al. 2010] A. M. Khludnev, J. Sokołowski, and K. Szulc, "Shape and topological sensitivity analysis in domains with cracks", Appl. Math. 55:6 (2010), 433-469.

[Koch and Zuazua 2006] H. Koch and E. Zuazua, "A hybrid system of PDE's arising in multistructure interaction: coupling of wave equations in $n$ and $n-1$ space dimensions", pp. 55-77 in Recent trends in partial differential equations (Santander, 2004), edited by J. L. Vázquez et al., Contemp. Math. 409, Amer. Math. Soc., Providence, RI, 2006. 
[Kovtunenko 2003] V. A. Kovtunenko, "Invariant energy integrals for a nonlinear crack problem with possible contact of the crack faces", Prikl. Mat. Mekh. 67:1 (2003), 109-123.

[Kozlov and Maz'ya 1991] V. A. Kozlov and V. G. Maz'ya, "On stress singularities near the boundary of a polygonal crack", Proc. Roy. Soc. Edinburgh Sect. A 117:1-2 (1991), 31-37.

[Nassar and Hassen 1987] M. Nassar and A. Hassen, "Embedded beam under equivalent load induced from a surface moving load", Acta Mech. 67 (1987), 237-247.

[Nazarov and Plamenevsky 1994] S. A. Nazarov and B. A. Plamenevsky, Elliptic problems in domains with piecewise smooth boundaries, De Gruyter Expositions in Mathematics 13, De Gruyter, Berlin, 1994.

[Neustroeva 2009] N. V. Neustroeva, “Односторонний контакт упругих пластин с жестким включением”, Vestn. Novosib. Gos. Univ. Ser. Mat. Mekh. Inform. 9:4 (2009), 51-64.

[Rotanova 2011] Т. A. Rotanova, "Задача об одностороннем контакте двух пластин, одна из которых содержит жесткое включение”, Vestn. Novosib. Gos. Univ. Ser. Mat. Mekh. Inform. 11:1 (2011), 87-98. Translated as "Unilateral contact problem for two plates with a rigid inclusion in the lower plate" in J. Math. Sci. 188:4 (2013), 452-462.

[Rudoy 2007] E. M. Rudoy, “Дифференцирование функционалов энергии в задаче о криволинейной трещине с возможным контактом берегов”, Izv. Akad. Nauk. Mekh. Tverd. Tela 2007:6 (2007), 113-127. Translated as "Differentiation of energy functionals in the problem on a curvilinear crack with possible contact between the shores" in Mech. Solids 42:6 (2007), 935-946.

[Rudoy 2011] E. M. Rudoy, “Асимптотика функционала энергии для упругого тела с трещиной и жестким включением: плоская задача”, Prikl. Mat. Mekh. 75:6 (2011), 1038-1048. Translated as "An asymptotic form of the energy functional for an elastic body with a crack and a rigid inclusion: the plane problem" in J. Appl. Math. Mech. 75:6 (2011), 731-738.

[Saccomandi and Beatty 2002] G. Saccomandi and M. F. Beatty, "Universal relations for fiberreinforced elastic materials", Math. Mech. Solids 7:1 (2002), 95-110.

Received 24 Jul 2012. Revised 19 Jan 2013. Accepted 25 Feb 2013.

AleXANDER M. KHLUDNEV: khlud@hydro.nsc.ru

Lavrentyev Institute of Hydrodynamics, Russian Academy of Sciences, Novosibirsk, 630090, Russia

GÜNTER R. LEUGERING: leugering@am.uni-erlangen.de

Institute of Applied Mathematics II, Friedrich-Alexander-Universität Erlangen-Nürnberg,

Cauerstrasse 11, D-91058 Erlangen, Germany 



\title{
INTERFACES ENDOWED WITH NONCONSTANT SURFACE ENERGIES REVISITED WITH THE D'ALEMBERT-LAGRANGE PRINCIPLE
}

\author{
HENRI GOUIN
}

\begin{abstract}
The equation of motion and the conditions on surfaces and edges between fluids and solids in the presence of nonconstant surface energies, as in the case of surfactants attached to fluid particles at the interfaces, are revisited under the principle of virtual work. We point out that adequate behaviors of surface concentrations may drastically modify the surface tension which naturally appears in the Laplace and the Young-Dupré equations. Thus, the principle of virtual work points out a strong difference between the two revisited concepts of surface energy and surface tension.
\end{abstract}

\section{Introduction}

This paper develops the principle of virtual work due to d'Alembert-Lagrange [Serrin 1959 $]^{1}$ when different phases of fluids are in contact through singular surfaces or interfaces. The study is first presented without a constitutive assumption for the surface energies, but the displacement fields are considered for a simple material corresponding to the first-gradient theory. The d'Alembert-Lagrange principle allows us to obtain the equation of motion and boundary conditions of mechanical nature and is able to be extended to more complex materials with microstructures [Daher and Maugin 1986] or to multigradient theories [Gouin 2007]. Here, we aim to emphasize the formulation of the principle of virtual work when the interfaces are endowed with nonconstant surface energies: the surfaces have their own material properties independent of the bulks and are embedded in the physical space, which is a three-dimensional metric space. The surface energy density is taken into account and naturally comes into the boundary conditions as the Laplace and the Young-Dupré equations by using variations associated with the virtual displacement fields. To do so, it is necessary to propose a constitutive equation of the surface energy; defining this is a main purpose of the paper. Such a

PACS2010: $02.30 . \mathrm{Xx}, 45.20 . \mathrm{dg}, 68.03 . \mathrm{Cd}, 68.35 . \mathrm{Gy}$.

Keywords: variational methods, capillarity, surface energy, surface tension.

${ }^{1}$ The principle of virtual work is also referred to in the literature as the principle of virtual power while virtual displacements are called virtual velocities [Germain 1973a; 1973b]. 
presentation is similar that of deformational and configurational mechanics [Steinmann 2008]; the method is analogous with the one employed in [Germain 1973a; 1973b; Daher and Maugin 1986] but with powerful differential geometry tools as in [Fosdick and Tang 2009]. However, the mathematical tools are adapted to the linear functional of virtual displacement fields and not to the integral balance laws over nonmaterial interfaces separating fluid phases as in [Cermelli et al. 2005].

Consequently, the main result of this paper is to propose a general form of the linear functional with interfaces in first-gradient theory which points out the significance of constitutive behaviors for the surface energies and highlights the clear difference between the notions of surface energy and surface tension. Fischer et al. [2008] emphasized a thermodynamical definition of surface energy, surface tension, and surface stress for which surface tension and surface stress are identical for fluids. Our presentation is not the same: without any thermodynamical assumptions, the difference between surface energy and surface tension is a natural consequence of the virtual work functional and the d'Alembert-Lagrange principle. The surface energy allows us to obtain the total energy of the interfaces and the surface tension is directly generated from the boundary conditions of the continuous medium.

In the simplest cases the two notions of surface energy and surface tension are mingled, but this is not generally the case when the surface energy is nonconstant along the interfaces. To prove this property, we first focus on the simplest case of Laplace's capillarity and obtain the well-known equations on interfaces and contact lines.

Surfaces endowed with surface matter, as in the case of surfactants, are a more complex case. Recent decades have seen the extension of surfactant applications in many fields including biology and medicine [Rosen 2004]; surfactants can also be expected to play a major mechanical role in the fluid and solid domains. The versatility of a surfactant mainly depends on its concentration at interfaces. It experimentally appears that a surfactant or surface-active agent is a substance present in liquids at a very low concentration rate and, when surface mass concentration is below the critical micelle concentration, it is mainly absorbed onto interfaces and alters only the interfacial free energies [de Gennes et al. 2004]. The interfacial free energy per unit area (generally called the surface energy) is the minimum amount of work required to create an interface at a given temperature [Edwards et al. 1991; Slattery et al. 2007]. The fact that surfactants can affect the mechanical behaviors of interfaces must be modelized in order to predict and control the properties of complete systems.

In fact, our aim is not to study the general case of surfactants proposed in the literature but to focus on the virtual work method to prove that simple behaviors of the surface energy depending on the mass concentration can drastically change the capillary effects. So, the concept of surface tension naturally appears in the 
equations on surfaces and on lines. In this paper, we call a surfactant the matter distributed only on the interfaces: we consider the special case when surfactant molecules are insoluble in the liquid bulk (the surface mass concentration is below the critical micelle concentration [Rosen 2004]) and are attached to fluid particles along the interfaces (without surface diffusion as in [McBride et al. 2011]).

The manuscript is organized as follows: Section 2 briefly reminds us of some results formally presenting the principle of virtual work in its more general form by using the kinematics of a continuous medium and the notion of virtual displacement. The simplest example of the Laplace model of capillarity concludes the section.

Section 3 deals with the case when the interfaces are endowed with nonconstant surface energy, whereby we essentially focus on liquid in contact with solid and gas. The special case of surfactants as interface matter attached to the fluid particles is considered. The surface energy depends on the surface matter concentration. Such a property drastically changes the boundary conditions on the interface by using surface tension instead of surface energy.

Section 4 deals with an explicit comparison between surface energy and surface tension only within deformational mechanics. And Section 5 is the conclusion in which some general extension can be forecast.

The main mathematics tools are collected in the large Appendix so that the presentation of the text is not cluttered with tedious calculations. The main mathematical tool is (15), which can be extended to more complex media.

\section{Virtual work for a continuous medium}

In continuum mechanics, motions can be equivalently studied with either the Newtonian model of a system offorces or the Lagrangian model of the work offorces [Germain 1973a; 1973b]. The Lagrangian model does not derive from a variational approach but, at equilibrium, the minimization of the energy coincides with the zero value of a linear functional. Generally, the linear functional expressing the work of forces is related to the theory of distributions; a decomposition theorem associated with displacements (as $C^{\infty}$-test functions whose supports are compact manifolds) uniquely determines a canonical zero order form (a separated form) with respect to both the test functions and the transverse derivatives of the contact test functions [Schwartz 1966]. In the same way that the Newtonian principle is useless when we do not have any constitutive equation for the system of forces, the d'AlembertLagrange principle is useless when we do not have any constitutive assumption for the virtual work functional.

The equation of motion and boundary conditions of a continuous medium derive from the d'Alembert-Lagrange principle of virtual work, which is an extension of the same principle in the mechanics of systems with a finite number of degrees of 
freedom: For any virtual displacement, the motion is such that the virtual work of forces is equal to the virtual work of mass accelerations [Gouin 2007].

2.1. The background of the principle of virtual work. The motion of a continuous medium is classically represented by a continuous transformation $\varphi$ of a threedimensional space into the physical set. In order to describe the transformation analytically, the variables $\boldsymbol{X}=\left(X^{1}, X^{2}, X^{3}\right)$ which single out individual particles correspond to material or Lagrange variables; the variables $\boldsymbol{x}=\left(x^{1}, x^{2}, x^{3}\right)$ correspond to Euler variables. The transformation representing the motion of a continuous medium is of the form

$$
\boldsymbol{x}=\boldsymbol{\varphi}(\boldsymbol{X}, t) \quad \text { or } \quad x^{i}=\varphi^{i}\left(X^{1}, X^{2}, X^{3}, t\right), \quad i \in\{1,2,3\},
$$

where $t$ denotes the time. At a fixed time the transformation possesses an inverse and continuous derivatives up to the second order except on singular surfaces, curves, or points. Then, the diffeomorphism $\varphi$ from the set $D_{0}$ of the particle references into the physical set $D$ is an element of a functional space $\wp$ of the positions of the continuous medium considered as a manifold with an infinite number of dimensions.

To formulate the d'Alembert-Lagrange principle of virtual work in continuum mechanics, we recall the notion of virtual displacements. This notion is obtained by letting the displacements arise from variations in the paths of particles. Let a one-parameter family of varied paths or virtual motions denoted by $\left\{\boldsymbol{\varphi}_{\eta}\right\}$, and possessing continuous partial derivatives up to the second order, be analytically expressed by the transformation

$$
\boldsymbol{x}=\boldsymbol{\Phi}(\boldsymbol{X}, t ; \eta),
$$

with $\eta \in O$, where $O$ is an open real set containing 0 , and such that $\boldsymbol{\Phi}(\boldsymbol{X}, t ; 0)=$ $\varphi(X, t)$ (the real motion of the continuous medium is obtained when $\eta=0$ ). The derivative with respect to $\eta$ at $\eta=0$ is denoted by $\delta$. In the literature, the derivative $\delta$ is named the variation and the virtual displacement is the variation of the position of the medium [Serrin 1959]. The virtual displacement is a tangent vector to $\wp$, the functional space of positions, at $\varphi\left(\delta \varphi \in T_{\varphi}(\wp)\right)$. In the physical space, the virtual displacement $\delta \varphi$ is determined by the variation of each particle: the virtual displacement $\zeta$ of the particle $\boldsymbol{x}$ is such that $\zeta=\delta \boldsymbol{x}$ when at $\eta=0,\{\delta \boldsymbol{X}=0, \delta t=0$, $\delta \eta=1\}$, and we associate the field of tangent vectors to $D$ :

$$
x \in D \quad \rightarrow \quad \zeta=\left.\psi(x) \equiv \frac{\partial \Phi}{\partial \eta}\right|_{\eta=0} \in T_{x}(D),
$$

where $T_{\boldsymbol{x}}(D)$ is the tangent vector bundle to $D$ at $\boldsymbol{x}$.

The virtual work concept, the dual of Newton's method, can be written in the following form: 

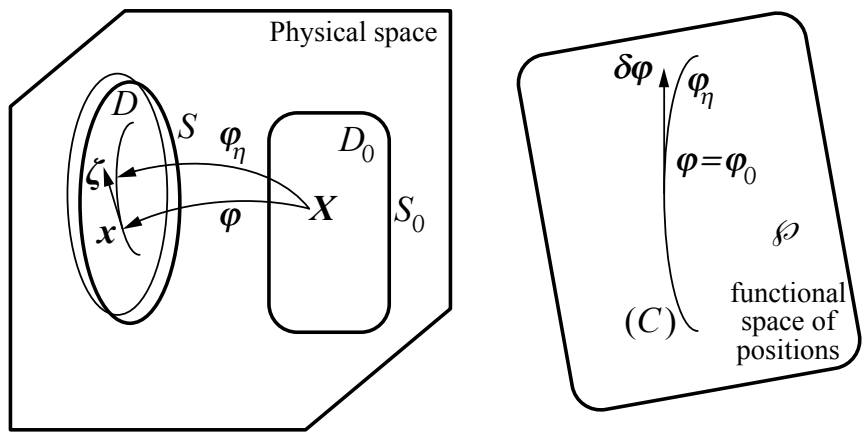

Figure 1. The boundary $S$ of $D$ is represented by a thick curve and its variation by a thin curve. The variation $\delta \varphi$ of the family $\left\{\varphi_{\eta}\right\}$ of varied paths belongs to $T_{\varphi}(\wp)$, tangent space of $\wp$ at $\varphi$.

The virtual work $\delta \tau$ is a linear functional value of the virtual displacement,

$$
\delta \tau=\langle\Im, \delta \varphi\rangle,
$$

where $\langle\cdot, \cdot\rangle$ denotes the inner product of $\Im$ and $\delta \varphi$, with $\Im$ belonging to the cotangent space $T_{\varphi}^{*}(\wp)$ of $\wp$ at $\varphi$.

In (3), the medium in position $\varphi$ is submitted to covector $\Im$ denoting all the "stresses" in mechanics. In the case of motion, we must add the inertial forces, corresponding to the accelerations of masses, to the volume forces.

The d'Alembert-Lagrange principle of virtual work is expressed as follows:

For all virtual displacements, the virtual work is null.

This principle leads to the analytic representation

$$
\forall \delta \varphi \in T_{\varphi}(\wp), \quad \delta \tau=0 .
$$

Theorem. If (3) is a distribution expressed in separated form (see [Schwartz 1966]), the d'Alembert-Lagrange principle yields the equation of motion and boundary conditions in the form $\mathfrak{\Im}=0$.

The virtual displacement is submitted to constraints coming from the constitutive equations and geometrical assumptions such as mass conservation. Consequently, the constraints are not expressed by Lagrange multipliers but are directly taken into account by the variations of the constitutive equations. The equation of motion and boundary conditions result from the explicit expression of $\delta \tau$ associated with the considered physical problem. As a first example, the simplest case of the theory of capillarity at equilibrium is considered. 
2.2. The classical Laplace theory of capillarity. Liquid-vapor and two-phase interfaces are represented by material surfaces endowed with an energy related to the Laplace free energy of capillarity. When working far from critical conditions, the capillary layer has a thickness equivalent to a few molecular beams [Ono and Kondo 1960; Domb 1996] and the interface appears as a geometrical surface separating the two media, with its own characteristic behavior and energy properties [Levitch 1962]. The domain $D$ of a compressible fluid (liquid) is immersed in a three Euclidean space. The boundary of the domain $D$ is a surface $S$ shared in $N$ parts $S_{p}$ of class $C^{2}, p=1, \ldots, N$ (Figure 2). We denote by $\left(R_{m}\right)^{-1}$ the mean curvature of $S$; the union of the limit edges $\Gamma_{p q}$ between surfaces $S_{p}$ and $S_{q}$ is assumed to be of class $C^{2}$ and $\boldsymbol{t}_{p}$ is the tangent vector to $\Gamma_{p}=\bigcup \Gamma_{p q}, q=1, \ldots, N$ with $q \neq p$, oriented by the unit external vector to $D$ denoted $\boldsymbol{n}_{p} ; \boldsymbol{n}_{p}^{\prime}=\boldsymbol{t}_{p} \times \boldsymbol{n}_{p}$ is the unit external normal vector to $\Gamma_{p}$ in the tangent plane to $S_{p}$; the edge $\Gamma$ of $S$ is the union of the edges $\Gamma_{p}$ of $S_{p}$.

To first verify the well-foundedness of the model, we consider the explicit expression of the functional $\delta \tau$ for compressible fluids with capillarity in the nondissipative case. The variation of the total energy $E$ of such a fluid results from the variation of the sum of the local density of energy integrated on the domain $D$ and the variation of the local density of surface energy integrated on its boundary $S$; to these variations, we must add the work of volume force $\rho \boldsymbol{f}$ in $D$, surface force $\boldsymbol{T}$ on $S$, and line force $\boldsymbol{L}$ on $\Gamma$. Such an amount represents, for the domain $D$, the virtual work of forces of the compressible fluid with capillarity.

The Laplace theory of capillarity introduces the notion of surface energy (or superficial energy) on surfaces such that, for a compressible liquid with capillary effects on the wall boundaries, the total energy of the fluid writes in the form

$$
E=\iiint_{D} \rho \alpha(\rho) d v+\iint_{S} \sigma d s, \quad \text { with } \iint_{S} \sigma d s \equiv \sum_{p=1}^{N} \iint_{S_{p}} \sigma_{p} d s
$$
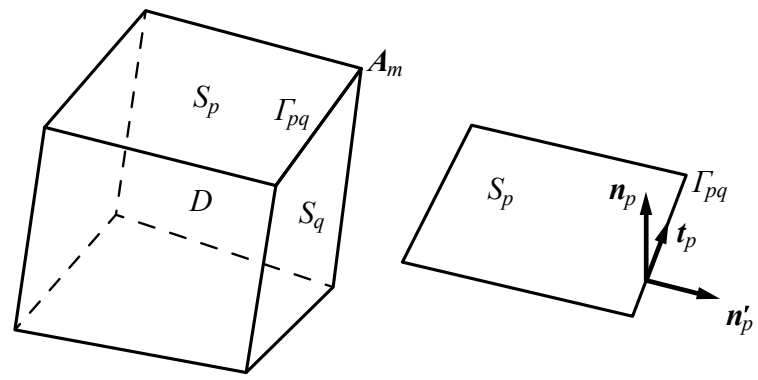

Figure 2. The set $D$ has a surface boundary $S$ divided into several parts. The edge of $S$ is denoted by $\Gamma$ which is also divided into several parts with endpoints $\boldsymbol{A}_{m}$. 
where $\rho$ is the matter density, $\alpha(\rho)$ is the fluid-specific energy ( $\rho \alpha(\rho)$ is the volume energy), and the coefficients $\sigma_{p}$ are the surface energy densities on each surface $S_{p}$ represented - for the sake of simplicity — by $\sigma$ on $S .^{2}$ Surface integrations are associated with the metric space. As proved in the Appendix, the variation of the deformation gradient tensor $F=\partial \boldsymbol{x} / \partial \boldsymbol{X}$ (with components $\left\{\partial x_{i} / \partial X_{j}\right\}$ ) of the mapping $\varphi$ combined with the mass conservation and the variation of $\sigma$ allow us to obtain the variation $-\delta E$ (see (A.3)); then the independent variables come from the position $\boldsymbol{x}$ of the continuous medium.

The virtual work of the volume forces defined on $D$ is generally in the form

$$
\iiint_{D} \rho \boldsymbol{f}^{T} \zeta d v, \quad \text { with } \boldsymbol{f}=-\operatorname{grad} U
$$

where $U(\boldsymbol{x})$ is a potential per unit mass and superscript $T$ denotes transposition. The virtual work of surface and line forces defined on $S$ and $\Gamma$ are, respectively,

$$
\iint_{S} \boldsymbol{T}^{T} \zeta d s \text { and } \quad \int_{\Gamma} \boldsymbol{L}^{T} \zeta d l
$$

Consequently, the total virtual work of forces $\delta \tau$ is

$$
\delta \tau=-\delta E+\iiint_{D} \rho \boldsymbol{f}^{T} \zeta d v+\iint_{S} \boldsymbol{T}^{T} \zeta d s+\int_{\Gamma} \boldsymbol{L}^{T} \zeta d l .
$$

From (A.3) and (A.6), we obtain

$$
\begin{aligned}
\delta \tau \equiv \iiint_{D}( & \left.-\operatorname{grad}^{T} p+\rho \boldsymbol{f}^{T}\right) \zeta d v+\int_{\Gamma}\left(\boldsymbol{L}^{T}-\sigma \boldsymbol{n}^{\prime T}\right) \zeta d l \\
& +\iint_{S}\left[-\delta \sigma+\left\{\left(p+\frac{2 \sigma}{R_{m}}\right) \boldsymbol{n}^{T}+\operatorname{grad}^{T} \sigma\left(\mathbf{1}-\boldsymbol{n n} \boldsymbol{n}^{T}\right)+\boldsymbol{T}^{T}\right\} \zeta\right] d s,
\end{aligned}
$$

where $p \equiv \rho^{2} \alpha^{\prime}(\rho)$ is the pressure of the liquid [Rocard 1952], $\delta \sigma$ denotes the variation of the surface energy $\sigma$, and $\mathbf{1}$ denotes the identity tensor. When $\sigma$ is constant we get $\delta \sigma=0$; then,

$$
\begin{aligned}
\delta \tau \equiv \iiint_{D}\left(-\operatorname{grad}^{T} p+\right. & \left.\rho \boldsymbol{f}^{T}\right) \zeta d v \\
& +\iint_{S}\left\{\left(p+\frac{2 \sigma}{R_{m}}\right) \boldsymbol{n}^{T}+\boldsymbol{T}^{T}\right\} \zeta d s+\int_{\Gamma}\left(\boldsymbol{L}^{T}-\sigma \boldsymbol{n}^{\prime T}\right) \zeta d l,
\end{aligned}
$$

and the d'Alembert-Lagrange principle yields the equation of equilibrium on $D$,

$$
-p_{, i}+\rho f_{i}=0 \quad \text { or } \quad-\operatorname{grad} p+\rho \boldsymbol{f}=0 .
$$

${ }^{2}$ Our aim is not to consider the thermodynamics of interfaces. Consequently, $\alpha$ and $\sigma$ are not considered as functions of thermodynamical variables such as temperature or entropy. 
The condition on boundary surface $S$ is

$$
\left(p+\frac{2 \sigma}{R_{m}}\right) n_{i}+T_{i}=0 \quad \text { or } \quad\left(p+\frac{2 \sigma}{R_{m}}\right) \boldsymbol{n}+\boldsymbol{T}=0,
$$

where, for an external fluid bordering $D, \boldsymbol{T}=-P \boldsymbol{n}$, with $P$ the value of the pressure in the external fluid. On the lines, it is necessary to consider the partition of $S$ such that the edge $\Gamma_{p q}$ is common to $S_{p}$ and $S_{q}$,

$$
\sigma_{p} n_{p i}^{\prime}+\sigma_{q} n_{q i}^{\prime}-L_{i}=0 \quad \text { or } \quad \sigma_{p} \boldsymbol{n}_{p}^{\prime}+\sigma_{q} \boldsymbol{n}_{q}^{\prime}-\boldsymbol{L}=0 .
$$

Surface condition (6) is the Laplace equation and line condition (7) is the YoungDupré equation with a line tension $\boldsymbol{L}$.

It is interesting to note that Steigmann and $\mathrm{Li}$ [1995] used the principle of virtual work by utilizing a system of line coordinates on boundary surfaces and lines. By introducing the free energy per unit area of interfaces and the free energy per unit of contact curve, they obtained Laplace's equation and a generalization of the YoungDupré equation of equilibrium; moreover, by employing the necessary conditions for energy-minimizing states of fluid systems they got a demonstration that the line tension associated with a three-phase contact curve must be nonnegative.

When $\sigma$ is not constant but $\delta \sigma=0$, we obtain the same equations for (5) and (7) but (6) on $S$ is replaced by

$$
\left(p+\frac{2 \sigma}{R_{m}}\right) \boldsymbol{n}+\left(\mathbf{1}-\boldsymbol{n} \boldsymbol{n}^{T}\right) \operatorname{grad} \sigma+\boldsymbol{T}=0 .
$$

The additive term $\left(\mathbf{1}-\boldsymbol{n} \boldsymbol{n}^{T}\right) \operatorname{grad} \sigma=\operatorname{grad}_{t g} \sigma$ is the tangential part of $\operatorname{grad} \sigma$ to the surface $S$. This term corresponds to a shear stress necessarily balanced by the tangential component of $\boldsymbol{T}$. Such is the case when $\sigma$ is defined on the $S_{0}$ image of $S$ in the reference space $D_{0}$ (then, $\sigma=\sigma_{0}(\boldsymbol{X})$ ). We understand the importance of the surface energy constitutive behavior; this questioning is emphasized in the following section.

\section{Capillarity of liquid in contact with solid and gas in the presence of nonconstant surface energy}

We have seen in the previous section that the problem associated with the behavior of the surface energy is the key point to obtaining the boundary conditions on interfaces and contact lines bordering the fluid bulk. In this section we consider a very special case of surfactant: the interfaces are endowed with a concentration of matter which affects the surface energy. The surface matter is attached to the particles of the fluid such that they obey together to the same equations of motion (1) and of virtual motion (2). We consider a more general case than in Section 2.2: 


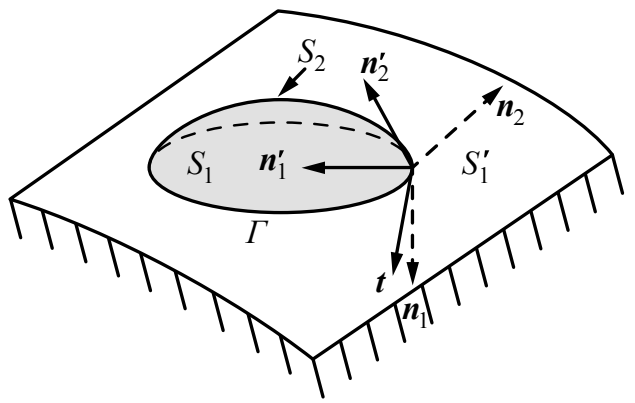

Figure 3. A liquid in drop form lies on a solid surface. The liquid is bordered by a gas and a solid; $S_{1}$ is the boundary between liquid and solid; $S_{1}^{\prime}$ is the boundary between gas and solid; $S_{2}$ is the interface between liquid and gas; $\boldsymbol{n}_{1}$ and $\boldsymbol{n}_{2}$ are the unit normal vectors to $S_{1}$ and $S_{2}$, external to the domain of the liquid; the edge $\Gamma$ (or contact line) is common to $S_{1}$ and $S_{2}$ and $t$ is the unit tangent vector to $\Gamma$ relative to $\boldsymbol{n}_{1} ; \boldsymbol{n}_{1}^{\prime}=\boldsymbol{n}_{1} \times \boldsymbol{t}$ and $\boldsymbol{n}_{2}^{\prime}=\boldsymbol{n}_{2} \times(-\boldsymbol{t})$ are the binormals to $\Gamma$ relative to $S_{1}$ and $S_{2}$, respectively.

we study the motion of the continuous medium with viscous forces. This viscosity affects not only the equation of motion but also the boundary conditions.

3.1. Geometrical description of the continuous medium. A drop of liquid fills the set $D$ and lies on the surface of a solid. The liquid drop is also bordered by a gas. All the interfaces between liquid, solid and gas are assumed to be regular surfaces. We call $\sigma_{S_{1}}$ and $\sigma_{S_{2}}$ the values of the surface energies of $S_{1}$ and $S_{2}$, respectively (see Figure 3). These energies may depend on each point of the boundary of $D$. Afterwards, on the domain $S_{1}^{\prime}$, the surface energy between gas and solid is neglected [Adamson 1967]. The liquid drop is submitted to a volume force $\rho \boldsymbol{f}$. The external surface force on $D$ is modelized with two constraint vector fields, $\boldsymbol{T}_{1}$ on the solid surface, $S_{1}$, and $\boldsymbol{T}_{2}$ on the free surface, $S_{2}$. The line tension $\boldsymbol{L}$ is assumed to be null.

By using the principle of virtual work, we aim to write the equation of motion of the liquid drop and the conditions on the surfaces and line bordering the liquid drop.

3.2. Surfactant attached to interfacial fluid particles. To express the behavior of the surface energy, we need to represent first the equation of the surface matter density.

Under the mapping $\varphi$, the set $D_{0}$ with boundary $S_{0}$ has image $D$ with boundary $S$. We assume there exists an insoluble surfactant with a surface mass concentration $c_{0}$ defined on $D_{0}$ of image $c$ in $D$ [Levitch 1962; Adamson 1967; Defay 1971]. 
Let us consider the case when the surfactant is attached to the fluid particles on the surface $S$, that is,

$$
c_{0}=c_{0}(\boldsymbol{X})
$$

where $\boldsymbol{X} \in S_{0}$.

The mass conservation of the surfactant on the surface $S$ requires that for any subset $S_{0}^{*}$ of $S_{0}$, of image $S^{*}$ subset of $S$,

$$
\iint_{S^{*}} c d s=\iint_{S_{0}^{*}} c_{0} d s_{0}
$$

Relation (9) implies

$$
c \operatorname{det} F \boldsymbol{n}_{0}^{T} F^{-1} \boldsymbol{n}=c_{0} \quad \text { with } \boldsymbol{n}_{0}^{T}=\frac{\boldsymbol{n}^{T} F}{\sqrt{\left(\boldsymbol{n}^{T} F F^{T} \boldsymbol{n}\right)}},
$$

where $\boldsymbol{n}_{0}$ denotes the unit normal vector to $S_{0}$. The proof of (10) is given in the Appendix.

From (10), we obtain the following: Firstly, the conservation of the surface concentration of the surfactant,

$$
\frac{d c}{d t}+c\left(\operatorname{div} \boldsymbol{u}-\boldsymbol{n}^{T} \boldsymbol{D} \boldsymbol{n}\right)=0,
$$

where $\boldsymbol{u}$ is the fluid velocity vector and

$$
\boldsymbol{D}=\frac{1}{2}\left(\partial \boldsymbol{u} / \partial \boldsymbol{x}+(\partial \boldsymbol{u} / \partial \boldsymbol{x})^{T}\right)
$$

denotes the rate of the deformation tensor of the fluid. The term $\operatorname{div} \boldsymbol{u}-\boldsymbol{n}^{T} \boldsymbol{D} \boldsymbol{n}$ expresses the tangential divergence relative to the surface $S$.

Secondly, the variation of the mass concentration of the surfactant,

$$
\delta c+c\left[\operatorname{div} \zeta-\boldsymbol{n}^{T} \frac{\partial \boldsymbol{\zeta}}{\partial \boldsymbol{x}} \boldsymbol{n}\right]=0 .
$$

The proofs of (11) and (12) are also given in the Appendix. In the case when the surface energy $\sigma$ is a function of the surfactant concentration,

$$
\sigma=\sigma(c),
$$

we deduce $\delta \sigma=\sigma^{\prime}(c) \delta c$. If we denote

$$
\gamma=\sigma-c \sigma^{\prime}(c)
$$

which is the Legendre transformation of $\sigma$ with respect to $c$, then by taking (A.3) into account we obtain, in the Appendix,

$$
\delta E=-\iint_{S}\left[\frac{2 \gamma}{R_{m}} \boldsymbol{n}^{T}+\operatorname{grad}^{T} \gamma\left(\mathbf{1}-\boldsymbol{n} \boldsymbol{n}^{T}\right)\right] \zeta d s+\int_{\Gamma} \gamma \boldsymbol{n}^{\prime T} \zeta d l .
$$


As we shall see in Section $4, \gamma$ is the surface tension of the interface $S$. The variation $\delta c$ of the concentration has an important consequence on the surfactant behavior and the surfactant behavior is essential to determining the virtual work of the liquid drop.

Relation (13) can easily be extended to several surfactants: if

$$
\sigma=\sigma\left(c_{1}, \ldots, c_{n}\right),
$$

where the $c_{i}$, for $i \in\{1, \ldots, n\}$, are the concentrations of the $n$ surfactants, then

$$
\gamma=\sigma-\sum_{i=1}^{n} c_{i} \frac{\partial \sigma}{\partial c_{i}},
$$

corresponding to the Legendre transformation of $\sigma$ with respect to $c_{i}, i \in\{1, \ldots, n\}$ and (14) is always valid.

3.3. Governing equation of motion and boundary conditions. As previously indicated, we do not consider the thermodynamical problem of interfaces, but, for example, when the medium is isothermal, $\alpha$ can be considered as the specific free energy of the bulk and $\sigma$ the free surface energy of the interface.

The use of virtual displacements yields a linear functional of virtual works, the sum of several partial works. To enumerate the works of forces, we have to consider how they are obtained in the literature [Germain 1973a; 1973b; Gouin 2007]. The virtual work expressions of volume force $\rho \boldsymbol{f}$, surface force $\boldsymbol{T}$, and liquid pressure $p$ are the same as in Section 2.2.

(a) For fluid motion, the virtual work of mass impulsions is

$$
-\iiint_{D} \rho \boldsymbol{a}^{T} \zeta d v
$$

where $\boldsymbol{a}$ is the acceleration vector.

(b) For dissipative motion, we must add the virtual work of viscous stresses

$$
-\iiint_{D} \operatorname{tr}\left(\boldsymbol{\tau}_{v} \frac{\partial \zeta}{\partial \boldsymbol{x}}\right) d v
$$

where $\boldsymbol{\tau}_{v}$ denotes the viscous stress tensor usually written in Navier-Stokes form [Adamson 1967]. Taking account of the relation

$$
\operatorname{tr}\left(\boldsymbol{\tau}_{v} \frac{\partial \zeta}{\partial \boldsymbol{x}}\right)=\operatorname{div}\left(\boldsymbol{\tau}_{v} \zeta\right)-\left(\operatorname{div} \boldsymbol{\tau}_{v}\right) \zeta
$$

an integration by parts using Stokes' formula in (14) for the virtual work of interfacial forces, together with the relations $\boldsymbol{n}_{1}^{\prime}=\boldsymbol{n}_{1} \times \boldsymbol{t}$ and $\boldsymbol{n}_{2}^{\prime}=-\boldsymbol{n}_{2} \times \boldsymbol{t}$, allows us 
to obtain the virtual work of forces applied to the domain $D$ :

$$
\begin{aligned}
\delta \tau & =\iiint_{D}\left(-\operatorname{grad}^{T} p+\operatorname{div} \boldsymbol{\tau}_{v}+\rho \boldsymbol{f}^{T}-\rho \boldsymbol{a}^{T}\right) \zeta d v \\
& +\iint_{S_{1}}\left[\operatorname{grad}^{T} \gamma_{1}\left(\mathbf{1}-\boldsymbol{n}_{1} \boldsymbol{n}_{1}^{T}\right)+\left(p+\frac{2 \gamma_{1}}{R_{m_{1}}}\right) \boldsymbol{n}_{1}^{T}-\boldsymbol{n}_{1}^{T} \boldsymbol{\tau}_{v}+\boldsymbol{T}_{1}^{T}\right] \zeta d s \\
& +\iint_{S_{2}}\left[\operatorname{grad}^{T} \gamma_{2}\left(\mathbf{1}-\boldsymbol{n}_{2} \boldsymbol{n}_{2}^{T}\right)+\left(p+\frac{2 \gamma_{2}}{R_{m_{2}}}\right) \boldsymbol{n}_{2}^{T}-\boldsymbol{n}_{2}^{T} \boldsymbol{\tau}_{v}+\boldsymbol{T}_{2}^{T}\right] \zeta d s \\
& +\int_{\Gamma}\left(\gamma_{1} \boldsymbol{n}_{1}^{\prime T}-\gamma_{2} \boldsymbol{n}_{2}^{\prime T}\right) \zeta d l,
\end{aligned}
$$

where $R_{m_{i}}$ denotes the mean radius of curvature of $S_{i}, \gamma_{i}$ denotes the surface tension of $S_{i}$, and $\boldsymbol{T}_{i}$ the surface force on $S_{i}, i \in\{1,2\} ; \boldsymbol{T}_{2}=-P \boldsymbol{n}_{2}$, where $P$ is the pressure in the external gas to the domain $D$.

The field of virtual displacement $x \in D \longrightarrow \zeta(x) \in T_{x}(D)$ must be tangent to the solid (rigid) surface $S_{1}$. The fundamental lemma of variational calculus yields the equation of motion associated with domain $D$, the conditions on surfaces $S_{1}$ and $S_{2}$, and the condition on contact line $\Gamma$.

Due to the fact that (15) is expressed in separate form in the sense of distributions [Schwartz 1966], the d'Alembert-Lagrange principle implies that $\forall \boldsymbol{\zeta}(\boldsymbol{x}) \in T_{x}(D)$ tangent to $S_{1}$, each of the four integrals of (15) is null. Then, we obtain equations on $D, S_{1}, S_{2}$, and $\Gamma$, respectively.

- We get the equation of motion in $D$ :

$$
\rho \boldsymbol{a}+\operatorname{grad} p=\left(\operatorname{div} \boldsymbol{\tau}_{v}\right)^{T}+\rho \boldsymbol{f} .
$$

Equation (16) is the Navier-Stokes equation for compressible fluids when $\boldsymbol{\tau}_{v}$ is written in the classical linear form by using the rate of the fluid deformation tensor, $\boldsymbol{\tau}_{v}=\lambda(\operatorname{tr} \boldsymbol{D}) \mathbf{1}+2 \mu \boldsymbol{D}$. We may add a classical condition for the velocity on the boundary as the adherence condition.

- We get the condition on surface $S_{1}$. The virtual displacement is tangent to $S_{1}$; the constraint $\boldsymbol{n}_{1}^{T} \zeta=0$ implies there exists a scalar Lagrange multiplier $\boldsymbol{x} \in S_{1} \longrightarrow$ $\chi(\boldsymbol{x}) \in \Re$, such that

$$
\left(p+\frac{2 \gamma_{1}}{R_{m_{1}}}\right) \boldsymbol{n}_{1}-\boldsymbol{\tau}_{v} \boldsymbol{n}_{1}+\left(\mathbf{1}-\boldsymbol{n}_{1} \boldsymbol{n}_{1}^{T}\right) \operatorname{grad} \gamma_{1}+\boldsymbol{T}_{1}=\chi \boldsymbol{n}_{1},
$$

The normal and tangential components of (17) relative to $S_{1}$ are deduced from (17):

$$
\begin{array}{r}
p+\frac{2 \gamma_{1}}{R_{m_{1}}}-\boldsymbol{n}_{1}^{T} \boldsymbol{\tau}_{v} \boldsymbol{n}_{1}+\boldsymbol{n}_{1}^{T} \boldsymbol{T}_{1}=\chi, \\
\left(\boldsymbol{1}-\boldsymbol{n}_{1} \boldsymbol{n}_{1}^{T}\right)\left(-\boldsymbol{\tau}_{v} \boldsymbol{n}_{1}+\operatorname{grad} \gamma_{1}+\boldsymbol{T}_{1}\right)=0 .
\end{array}
$$


Following (18), we obtain the value of $\chi$ along the surface $S_{1}$. The scalar field $\chi$ corresponds to the unknown value of the normal stress vector on the surface $S_{1}$; it corresponds to the difference between the mechanical and viscous normal stresses and a stress due to the curvature of $S_{1}$ taking into account the surface tension. Equation (19) represents the balance between the tangential components of the mechanical and viscous stresses and the tangential component of the surface tension gradient.

- We get the condition on surface $S_{2}$ :

$$
\left(p+\frac{2 \gamma_{2}}{R_{m_{2}}}\right) \boldsymbol{n}_{2}-\boldsymbol{\tau}_{v} \boldsymbol{n}_{2}+\left(\mathbf{1}-\boldsymbol{n}_{2} \boldsymbol{n}_{2}^{T}\right) \operatorname{grad} \gamma_{2}-P \boldsymbol{n}_{2}=0 .
$$

The normal and tangential components of (20) relative to $S_{2}$ are deduced:

$$
\begin{aligned}
\frac{2 \gamma_{2}}{R_{m_{2}}}-\boldsymbol{n}_{2}^{T} \boldsymbol{\tau}_{v} \boldsymbol{n}_{2}+p & =P, \\
\left(\mathbf{1}-\boldsymbol{n}_{2} \boldsymbol{n}_{2}^{T}\right)\left(-\boldsymbol{\tau}_{v} \boldsymbol{n}_{2}+\operatorname{grad} \gamma_{2}\right) & =0 .
\end{aligned}
$$

Equation (21) corresponds to the expression of the Laplace equation in the case of viscous motion; the normal component of viscous stresses is taken into account. Equation (22) is similar to (19) for the surface $S_{2}$ but without the component of the stress vector.

- We get the condition on line $\Gamma$. To get the line condition we must consider a virtual displacement tangent to $S_{1}$ and consequently in the form

$$
\zeta=\alpha \boldsymbol{t}+\beta \boldsymbol{t} \times \boldsymbol{n}_{1},
$$

where $\alpha$ and $\beta$ are two scalar fields defined on $\Gamma$. From the last integral of (15), we get the following immediately: For any scalar field $\boldsymbol{x} \in \Gamma \longrightarrow \beta(\boldsymbol{x}) \in \Re$,

$$
\int_{\Gamma} \beta \gamma_{1} \boldsymbol{n}_{1}^{\prime T}\left(\boldsymbol{t} \times \boldsymbol{n}_{1}\right) d l-\int_{\Gamma} \beta \gamma_{2} \boldsymbol{n}_{2}^{\prime T}\left(\boldsymbol{t} \times \boldsymbol{n}_{1}\right) d l=0,
$$

with $\boldsymbol{n}_{1}^{\prime}=-\boldsymbol{t} \times \boldsymbol{n}_{1}$ and $\boldsymbol{n}_{2}^{\prime}=\boldsymbol{t} \times \boldsymbol{n}_{2}$, and consequently,

$$
-\gamma_{1}-\gamma_{2} \boldsymbol{n}_{2}^{T} \boldsymbol{n}_{1}=0
$$

Denoting by $\theta$ the angle $\left\langle\boldsymbol{n}_{1}, \boldsymbol{n}_{2}\right\rangle$, we obtain the well-known relation of YoungDupré but adapted to $\gamma_{1}$ and $\gamma_{2}$ in place of $\sigma_{1}$ and $\sigma_{2}$ :

$$
\gamma_{1}+\gamma_{2} \cos \theta=0 \text {. }
$$

3.4. Remarks. For a motionless fluid, $\boldsymbol{\tau}_{v}=0$ and consequently, (19) yields

$$
\operatorname{grad}_{t g} \gamma_{1}=-\boldsymbol{T}_{1 t g},
$$


where $\operatorname{grad}_{t g} \gamma_{1}$ and $\boldsymbol{T}_{1 t g}$ denote the tangential parts of $\operatorname{grad} \gamma_{1}$ and $\boldsymbol{T}_{1}$, respectively. The tangential part of the vector stress is opposite to the surface tension gradient. Therefore, at a given value of $T_{1 n}=\boldsymbol{n}_{1}^{T} \boldsymbol{T}_{1}$, (18) yields the value $\chi$ corresponding to the normal stress vector to the surface $S_{1}$, (21) yields $P=p+2 \gamma_{2} / R_{m_{2}}$ corresponding to the classical equation of Badshforth and Adams [Adamson 1967] but with the surface tension $\gamma_{2}$ instead of $\sigma_{2}$, and (22) implies $\left(\mathbf{1}-\boldsymbol{n}_{2} \boldsymbol{n}_{2}^{T}\right) \operatorname{grad} \gamma_{2}=0$. At equilibrium, along $S_{2}$, the surface tension $\gamma_{2}$ must be uniform.

In the case of motion, (22) represents the Marangoni effect as proposed in [Gibbs 1928; Defay 1971] but with the surface tension $\gamma_{2}$ instead of $\sigma_{2}$.

\section{Surface energy and surface tension}

A surface tension must appear on the boundary conditions as a force per unit of line. The Legendre transformation $\gamma$ of $\sigma$ with respect to $c$ exactly corresponds to this property on the contact line $\Gamma$; then, surface tension $\gamma$ differs from the surface energy; this important property was pointed out by Gibbs [1928] and Defay [1971] by means of thermodynamical considerations. The fundamental difference between surface tension and surface energy, in the presence of attached surfactants, is illustrated in the following cases corresponding to formal behaviors.

- If $\sigma$ is independent of $c$, then $\gamma=\sigma$ : the surface tension is equal to the surface energy. This is the classical case of capillarity for fluids considered in Section 2.2 and (23) is the classical Young-Dupré condition on the contact lines.

- In fact, $\sigma$ is a decreasing function of $c$ [Adamson 1967]; when $c$ is small enough we consider the behavior

$$
\sigma=\sigma_{0}-\sigma_{1} c, \quad \text { where } \sigma_{0}>0 \text { and } \sigma_{1}>0 ;
$$

then, (13) implies $\gamma=\sigma_{0}$ and surface tension and surface energy are different.

- Now, we consider a formal case when the surface energy density model is written in the form

$$
\sigma=\sigma_{0}-\sigma_{1} c-\sigma_{2} c \sin \left(\frac{1}{c}\right)
$$

where $\sigma_{0}>0, \sigma_{1}>0$, and $\sigma_{2}>0$. Then, (13) implies

$$
\gamma=\sigma_{0}-\sigma_{2} \cos \left(\frac{1}{c}\right) \text {. }
$$

This case does not correspond to $\sigma$ as a monotonic decreasing function of $c$. Nevertheless, when $c \rightarrow 0, \gamma$ does not have any limit and we get

$$
\gamma \in\left[\sigma_{0}-\sigma_{2}, \sigma_{0}+\sigma_{2}\right] .
$$

The surface tension may have a large scale of values. When the concentration $c$ is low, a variation of the concentration $c$ may generate strong fluctuations of 
the surface tension without significant change of the surface energy. Alternatively, the concentration behavior strongly affects the surface tension but not the surface energy. Relation (24) fits with the well-known physical case of hysteresis behavior for a drop lying on a horizontal plane (see, for example, [Gouin 2003] and the literature therein). So, the surface roughness is not the only reason of the hysteresis of the contact angle even if the surface energy is nearly constant.

\section{Conclusion}

The principle of virtual work allows us to deduce the equation of motion and the conditions on the surfaces and lines by means of a variational analysis. When capillary forces operate and surfactant molecules are attached to the fluid molecules at the interfaces, the conditions on the surfaces and lines point out a fundamental difference between the concepts of surface energy and surface tension. This fact was thermodynamically predicted in [Gibbs 1928; Defay 1971]. The hysteresis phenomenon may appear even if surface energy is almost constant on a planar substrate when the surface tension strongly varies.

In (23), $\gamma_{1}$ and $\gamma_{2}$ are not assumed to be constant, but are defined at each point of $\Gamma$. This expression of the Young-Dupré boundary condition on the contact line $\Gamma$ is not true in more complex cases. For example, in the case when the surface tension is a nonlocal functional of surfactant concentration, the surface tension is no longer the classical Legendre transformation of the surface energy relative to surfactant concentration and more complex behaviors can be foreseen. These behaviors can change the variation of the integral of the free energy as in the case of shells or in second-gradient models for which boundary conditions become more complex [Cosserat and Cosserat 1909; Toupin 1962; Germain 1973b; Noll and Virga 1990; Dell'Isola and Seppecher 1997]. In a further article [Gouin $\geq 2014$ ], we will see this is also the case when the surface energy depends on the surface curvature as in membranes and vesicles [Helfrich 1973; Seifert 1997; Agrawal and Steigmann 2011].

\section{Appendix: Geometrical preliminaries}

[Kobayashi and Nomizu 1963; Aris 1989; Gouin and Kosiński 1998]

A.1 Expression of the virtual work of forces in capillarity. The hypotheses and notations are presented in Section 2.2.

Lemma 1. We have the following relations:

$$
\begin{aligned}
\delta \operatorname{det} F & =\operatorname{det} F \operatorname{div} \zeta, \\
\delta\left(F^{-1} \boldsymbol{n}\right) & =-F^{-1} \frac{\partial \zeta}{\partial \boldsymbol{x}} \boldsymbol{n}+F^{-1} \delta \boldsymbol{n} .
\end{aligned}
$$


The proof of (A.1) comes from the so-called Jacobi identity,

$$
\delta(\operatorname{det} F)=\operatorname{det} F \operatorname{tr}\left(F^{-1} \delta F\right),
$$

and from

$$
\delta F=\delta\left(\frac{\partial \boldsymbol{x}}{\partial \boldsymbol{X}}\right)=\frac{\partial \zeta}{\partial \boldsymbol{X}}
$$

Then,

$$
\operatorname{tr}\left(F^{-1} \delta F\right)=\operatorname{tr}\left(\frac{\partial \boldsymbol{X}}{\partial \boldsymbol{x}} \frac{\partial \zeta}{\partial \boldsymbol{X}}\right)=\operatorname{tr}\left(\frac{\partial \zeta}{\partial \boldsymbol{X}} \frac{\partial \boldsymbol{X}}{\partial \boldsymbol{x}}\right)=\operatorname{tr}\left(\frac{\partial \zeta}{\partial \boldsymbol{x}}\right)=\operatorname{div} \zeta
$$

The proof of (A.2) comes as follows:

$$
\delta\left(F^{-1} \boldsymbol{n}\right)=\delta\left(F^{-1}\right) \boldsymbol{n}+F^{-1} \delta \boldsymbol{n} .
$$

But $F^{-1} F=\mathbf{1}$ implies

$$
\delta\left(F^{-1}\right) F+F^{-1} \delta F=0,
$$

and so also

$$
\delta\left(F^{-1}\right)=-F^{-1} \frac{\partial \zeta}{\partial \boldsymbol{X}} F^{-1}=-F^{-1} \frac{\partial \zeta}{\partial \boldsymbol{x}},
$$

which yields (A.2).

Lemma 2. Let us consider the surface integral

$$
E=\iint_{S} \sigma d s
$$

Then the variation of $E$ is,

$$
\delta E=\iint_{S}\left[\delta \sigma-\left(\frac{2 \sigma}{R_{m}} \boldsymbol{n}^{T}+\operatorname{grad}^{T} \sigma\left(\mathbf{1}-\boldsymbol{n} \boldsymbol{n}^{T}\right)\right) \zeta\right] d s+\int_{\Gamma} \sigma \boldsymbol{n}^{\prime T} \zeta d l .
$$

Relation (A.3) points out the extreme importance of knowing the variation of $\delta \sigma$. The variation $\delta E$ of $E$ drastically changes following the different possible behaviors of the surface energy.

The proof can be found as follows. The external normal $\boldsymbol{n}(\boldsymbol{x})$ to $S$ is locally extended in the vicinity of $S$ by the relation $\boldsymbol{n}(\boldsymbol{x})=\operatorname{grad} d(\boldsymbol{x})$, where $d$ is the distance of point $\boldsymbol{x}$ to $S$; for any vector field $\boldsymbol{w}$, we obtain [Kobayashi and Nomizu 1963; Aris 1989]

$$
\operatorname{rot}(\boldsymbol{n} \times \boldsymbol{w})=\boldsymbol{n} \operatorname{div} \boldsymbol{w}-\boldsymbol{w} \operatorname{div} \boldsymbol{n}+\frac{\partial \boldsymbol{n}}{\partial \boldsymbol{x}} \boldsymbol{w}-\frac{\partial \boldsymbol{w}}{\partial \boldsymbol{x}} \boldsymbol{n} .
$$

From $\boldsymbol{n}^{T} \frac{\partial \boldsymbol{n}}{\partial \boldsymbol{x}}=0$ and $\operatorname{div} \boldsymbol{n}=-\frac{2}{R_{m}}$, we deduce on $S$,

$$
\boldsymbol{n}^{T} \operatorname{rot}(\boldsymbol{n} \times \boldsymbol{w})=\operatorname{div} \boldsymbol{w}+\frac{2}{R_{m}} \boldsymbol{n}^{T} \boldsymbol{w}-\boldsymbol{n}^{T} \frac{\partial \boldsymbol{w}}{\partial \boldsymbol{x}} \boldsymbol{n} .
$$


Due to

$$
E=\iint_{S} \sigma \operatorname{det}\left(\boldsymbol{n}, d_{1} \boldsymbol{x}, d_{2} \boldsymbol{x}\right),
$$

where $d_{1} \boldsymbol{x}$ and $d_{2} \boldsymbol{x}$ are differential vectors associated with two coordinate lines of $S$, we get

$$
E=\iint_{S_{0}} \sigma \operatorname{det} F \operatorname{det}\left(F^{-1} \boldsymbol{n}, d_{1} \boldsymbol{X}, d_{2} \boldsymbol{X}\right),
$$

where $d_{1} \boldsymbol{x}=F d_{1} \boldsymbol{X}$ and $d_{2} \boldsymbol{x}=F d_{2} \boldsymbol{X}$. Then,

$\delta E=\iint_{S_{0}} \delta \sigma \operatorname{det} F \operatorname{det}\left(F^{-1} \boldsymbol{n}, d_{1} \boldsymbol{X}, d_{2} \boldsymbol{X}\right)+\iint_{S_{0}} \sigma \delta\left(\operatorname{det} F \operatorname{det}\left(F^{-1} \boldsymbol{n}, d_{1} \boldsymbol{X}, d_{2} \boldsymbol{X}\right)\right)$.

Due to Lemma 1 and the fact that $\boldsymbol{n}^{T} \boldsymbol{n}=1$ implies $\boldsymbol{n}^{T} \delta \boldsymbol{n}=0$,

$$
\begin{aligned}
& \iint_{S_{0}} \sigma \delta\left(\operatorname{det} F \operatorname{det}\left(F^{-1} \boldsymbol{n}, d_{1} \boldsymbol{X}, d_{2} \boldsymbol{X}\right)\right) \\
& =\iint_{S}\left[\sigma \operatorname{div} \zeta \operatorname{det}\left(\boldsymbol{n}, d_{1} \boldsymbol{x}, d_{2} \boldsymbol{x}\right)+\sigma \operatorname{det}\left(\delta \boldsymbol{n}, d_{1} \boldsymbol{x}, d_{2} \boldsymbol{x}\right)-\sigma \operatorname{det}\left(\frac{\partial \zeta}{\partial \boldsymbol{x}} \boldsymbol{n}, d_{1} \boldsymbol{x}, d_{2} \boldsymbol{x}\right)\right] \\
& =\iint_{S}\left(\operatorname{div}(\sigma \zeta)-\left(\operatorname{grad}^{T} \sigma\right) \zeta-\sigma \boldsymbol{n}^{T} \frac{\partial \zeta}{\partial \boldsymbol{x}} \boldsymbol{n}\right) d s .
\end{aligned}
$$

Relation (A.4) yields

$$
\operatorname{div}(\sigma \zeta)+\frac{2 \sigma}{R_{m}} \boldsymbol{n}^{T} \zeta-\boldsymbol{n}^{T} \frac{\partial \sigma \zeta}{\partial \boldsymbol{x}} \boldsymbol{n}=\boldsymbol{n}^{T} \operatorname{rot}(\sigma \boldsymbol{n} \times \zeta)
$$

Then,

$$
\begin{aligned}
\iint_{S_{0}} \sigma \delta\left(\operatorname{det} F \operatorname{det}\left(F^{-1} \boldsymbol{n}, d_{1} \boldsymbol{X}, d_{2} \boldsymbol{X}\right)\right) \\
=\iint_{S}\left(-\frac{2 \sigma}{R_{m}} \boldsymbol{n}^{T}+\operatorname{grad}^{T} \sigma\left(\boldsymbol{n} \boldsymbol{n}^{T}-\mathbf{1}\right)\right) \zeta d s+\iint_{S} \boldsymbol{n}^{T} \operatorname{rot}(\sigma \boldsymbol{n} \times \zeta) d s,
\end{aligned}
$$

where $\operatorname{grad}^{T} \sigma\left(\boldsymbol{n} \boldsymbol{n}^{T}-\mathbf{1}\right)$ belongs to the cotangent plane to $S$ and we obtain (A.3).

A.1.1 Variation of the internal energy. Let us note that

$$
\delta \iiint_{D} \rho \alpha d v=\iiint_{D} \rho \delta \alpha d v, \quad \text { where } \delta \alpha=\frac{\partial \alpha}{\partial \rho} \delta \rho .
$$

Due to the mass conservation,

$$
\rho \operatorname{det} F=\rho_{0}(\boldsymbol{X}),
$$

where $\rho_{0}$ is defined on $D_{0}$, the differentiation of (A.5) yields

$$
\delta \rho \operatorname{det} F+\rho \delta(\operatorname{det} F)=0,
$$


and from Lemma 1 we get

$$
\delta \rho=-\rho \operatorname{div} \zeta .
$$

Consequently, from $p=\rho^{2} \frac{\partial \alpha}{\partial \rho}$ and $\operatorname{div}(p \zeta)=p \operatorname{div} \zeta+(\operatorname{grad} p)^{T} \zeta$, we get

$$
\begin{aligned}
\delta \iiint_{D} \rho \alpha d v & =\iiint_{D} \rho \frac{\partial \alpha}{\partial \rho} \delta \rho d v=\iiint_{D}-p \operatorname{div} \zeta d v \\
& =\iiint_{D}(\operatorname{grad} p)^{T} \zeta d v-\iint_{S} p \boldsymbol{n}^{T} \zeta d s .
\end{aligned}
$$

By taking into account (A.3), we immediately get (4).

\section{A.2 Study of a surfactant attached to fluid particles.}

A.2.1 Proof of (10). Under the hypotheses and notations of Section 3.2,

$$
\begin{aligned}
\iint_{S^{*}} c d s & =\iint_{S^{*}} \operatorname{det}\left(\boldsymbol{n} c, d_{1} \boldsymbol{x}, d_{2} \boldsymbol{x}\right)=\iint_{S_{0}^{*}} \operatorname{det}\left(F F^{-1} \boldsymbol{n} c, F d_{1} \boldsymbol{X}, F d_{2} \boldsymbol{X}\right) \\
& =\iint_{S_{0}^{*}} c(\operatorname{det} F) \operatorname{det}\left(F^{-1} \boldsymbol{n}, d_{1} \boldsymbol{X}, d_{2} \boldsymbol{X}\right)=\iint_{S_{0}^{*}} c(\operatorname{det} F) \boldsymbol{n}_{0}^{T} F^{-1} \boldsymbol{n} d s_{0},
\end{aligned}
$$

where $\boldsymbol{n}_{0}^{T} \boldsymbol{n}_{0}=1$. Moreover, $\boldsymbol{n}^{T} d \boldsymbol{x}=0 \Rightarrow \boldsymbol{n}^{T} F d \boldsymbol{X}=0$, then $\boldsymbol{n}_{0}^{\prime T}=\boldsymbol{n}^{T} F$ is normal to $S_{0}^{*}$, and consequently,

$$
\boldsymbol{n}_{0}^{T}=\frac{\boldsymbol{n}^{T} F}{\sqrt{\left(\boldsymbol{n}^{T} F F^{T} \boldsymbol{n}\right)}}, \quad \boldsymbol{n}^{T}=\frac{\boldsymbol{n}_{0}^{T} F^{-1}}{\sqrt{\left(\boldsymbol{n}_{0}^{T} F^{-1}\left(F^{-1}\right)^{T} \boldsymbol{n}_{\mathbf{0}}\right)}},
$$

and from (9),

$$
c \operatorname{det} F \sqrt{\boldsymbol{n}_{0}^{T} F^{-1}\left(F^{-1}\right)^{T} \boldsymbol{n}_{0}}=c_{0} .
$$

A.2.2 Proof of (11) and (12). With the notations of Section 3.2, (A.7) yields

$$
\frac{d c}{d t}=-\frac{c_{0} \frac{d(\operatorname{det} F)}{d t}}{(\operatorname{det} F)^{2} \sqrt{\left(\boldsymbol{n}_{0}^{T} F^{-1}\left(F^{-1}\right)^{T} \boldsymbol{n}_{0}\right)}}-\frac{c_{0} \frac{d}{d t}\left(\boldsymbol{n}_{0}^{T} F^{-1}\left(F^{-1}\right)^{T} \boldsymbol{n}_{0}\right)}{2 \operatorname{det} F\left(\boldsymbol{n}_{0}^{T} F^{-1}\left(F^{-1}\right)^{T} \boldsymbol{n}_{0}\right)^{3 / 2}} .
$$

But, $\frac{d(\operatorname{det} F)}{d t}=(\operatorname{det} F) \operatorname{div} \boldsymbol{u}$ and $\frac{d}{d t}\left(F^{-1}\left(F^{-1}\right)^{T}\right)=-2 F^{-1} \boldsymbol{D}\left(F^{-1}\right)^{T}$. Then,

$$
\frac{d c}{d t}+c\left(\operatorname{div} \boldsymbol{u}-\boldsymbol{n}^{T} \boldsymbol{D} \boldsymbol{n}\right)=0 .
$$

The same calculation with $\delta$ in place of $\frac{d}{d t}$ yields immediately

$$
\delta c+c\left[\operatorname{div} \zeta-\boldsymbol{n}^{T} \frac{\partial \zeta}{\partial \boldsymbol{x}} \boldsymbol{n}\right]=0
$$


A.2.3 Proof of (14). From (12) and $\sigma=\sigma(c)$ we get

$$
\delta \sigma=\kappa\left[\operatorname{div} \zeta-\boldsymbol{n}^{T} \frac{\partial \zeta}{\partial \boldsymbol{x}} \boldsymbol{n}\right], \quad \text { with } \kappa(c)=-c \sigma^{\prime}(c) .
$$

Consequently,

$$
\iint_{S} \delta \sigma=\iint_{S}\left(\operatorname{div}(\kappa \zeta)-\operatorname{grad}^{T} \kappa \zeta-\kappa \boldsymbol{n}^{T} \frac{\partial \zeta}{\partial \boldsymbol{x}} \boldsymbol{n}\right) d s
$$

But (A.4) implies

$$
\boldsymbol{n}^{T} \operatorname{rot}(\kappa \boldsymbol{n} \times \zeta)=\operatorname{div}(\kappa \zeta)+\frac{2 \kappa}{R_{m}} \boldsymbol{n}^{T} \zeta-\boldsymbol{n}^{T} \frac{\partial(\kappa \zeta)}{\partial \boldsymbol{x}} \boldsymbol{n},
$$

and

$$
\boldsymbol{n}^{T} \frac{\partial(\kappa \zeta)}{\partial \boldsymbol{x}} \boldsymbol{n}=\left(\boldsymbol{n}^{T} \zeta\right) \cdot\left(\operatorname{grad}^{T} \kappa \boldsymbol{n}\right)+\kappa \boldsymbol{n}^{T} \frac{\partial \zeta}{\partial \boldsymbol{x}} \boldsymbol{n}=\operatorname{grad}^{T} \kappa \boldsymbol{n} \boldsymbol{n}^{T} \zeta+\kappa \boldsymbol{n}^{T} \frac{\partial \zeta}{\partial \boldsymbol{x}} \boldsymbol{n} .
$$

Then,

$\operatorname{div}(\kappa \zeta)-\operatorname{grad}^{T} \kappa \zeta-\kappa \boldsymbol{n}^{T} \frac{\partial \zeta}{\partial \boldsymbol{x}} \boldsymbol{n}=-\frac{2 \kappa}{R_{m}} \boldsymbol{n}^{T} \zeta-\operatorname{grad}^{T} \kappa\left(\mathbf{1}-\boldsymbol{n} \boldsymbol{n}^{T}\right) \zeta+\boldsymbol{n}^{T} \operatorname{rot}(\kappa \boldsymbol{n} \times \zeta)$.

Due to

$$
\iint_{S} \boldsymbol{n}^{T} \operatorname{rot}(\kappa \boldsymbol{n} \times \zeta) d s=\int_{\Gamma} \kappa \boldsymbol{n}^{T} \zeta d l
$$

we get

$$
\iint_{S}-\delta \sigma d s=\iint_{S}\left[\frac{2 \kappa}{R_{m}} \boldsymbol{n}^{T}+\operatorname{grad}^{T} \kappa\left(\mathbf{1}-\boldsymbol{n} \boldsymbol{n}^{T}\right)\right] \zeta d s-\int_{\Gamma} \kappa \boldsymbol{n}^{\prime T} \zeta d l,
$$

and (A.3) yields

$$
\delta E=-\iint_{S}\left[\frac{2 \gamma}{R_{m}} \boldsymbol{n}^{T}+\operatorname{grad}^{T} \gamma\left(\mathbf{1}-\boldsymbol{n} \boldsymbol{n}^{T}\right)\right] \zeta d s+\int_{\Gamma} \gamma \boldsymbol{n}^{\prime T} \zeta d l .
$$

\section{Acknowledgements}

I am indebted to the three anonymous referees for much valuable criticism, comments, and suggestions during the review process.

\section{References}

[Adamson 1967] A. W. Adamson, Physical chemistry of surfaces, Interscience, New York, 1967.

[Agrawal and Steigmann 2011] A. Agrawal and D. J. Steigmann, "A model for surface diffusion of trans-membrane proteins on lipid bilayers", Z. Angew. Math. Phys. 62:3 (2011), 549-563.

[Aris 1989] R. Aris, Vectors, tensors, and the basic equations of fluid mechanics, Dover, New York, 1989. 
[Cermelli et al. 2005] P. Cermelli, E. Fried, and M. E. Gurtin, "Transport relations for surface integrals arising in the formulation of balance laws for evolving fluid interfaces", J. Fluid Mech. 544 (2005), 339-351.

[Cosserat and Cosserat 1909] E. Cosserat and F. Cosserat, Sur la théorie des corps déformables, Hermann, Paris, 1909.

[Daher and Maugin 1986] N. Daher and G. A. Maugin, "The method of virtual power in continuum mechanics. Application to media presenting singular surfaces and interfaces", Acta Mech. 60:3-4 (1986), 217-240.

[Defay 1971] R. Defay, Thermodynamique de la tension superficielle, Gauthier-Villars, Paris, 1971.

[Dell'Isola and Seppecher 1997] F. dell'Isola and P. Seppecher, "Edge contact forces and quasibalanced power", Meccanica 32:1 (1997), 33-52.

[Domb 1996] C. Domb, The critical point, Taylor \& Francis, London, 1996.

[Edwards et al. 1991] D. A. Edwards, H. Brenner, and D. T. Wasan, Interfacial transport processes and rheology, Butterworth-Heinemann, Stoneham, 1991.

[Fischer et al. 2008] F. D. Fischer, T. Waitz, D. Vollath, and N. K. Simha, "On the role of surface energy and surface stress in phase-transforming nanoparticles", Progress in Materials Science 53:3 (2008), 481-527.

[Fosdick and Tang 2009] R. Fosdick and H. Tang, "Surface transport in continuum mechanics", Mathematics and Mechanics of Solids 14:6 (2009), 587-598.

[de Gennes et al. 2004] P. G. de Gennes, F. Brochard-Wyart, and D. Quéré, Capillarity and wetting phenomena: drops, bubbles, pearls, waves, Springer, Berlin, 2004.

[Germain 1973a] P. Germain, "La méthode des puissances virtuelles en mécanique des milieux continus. I. Théorie du second gradient”, J. Mécanique 12 (1973), 235-274.

[Germain 1973b] P. Germain, "The method of the virtual power in continuum mechanics, 2: Microstructure", SIAM J. Appl. Math. 25 (1973), 556-575.

[Gibbs 1928] J. W. Gibbs, The scientific papers of J. Willard Gibbs, vol. 1, Longmans, London, 1928.

[Gouin 2003] H. Gouin, "The wetting problem of fluids on solid surfaces, II: The contact angle hysteresis”, Contin. Mech. Thermodyn. 15:6 (2003), 597-611.

[Gouin 2007] H. Gouin, "The d'Alembert-Lagrange principle for gradient theories and boundary conditions", pp. 79-95 in Asymptotic methods in nonlinear wave phenomena, World Sci. Publ., Hackensack, NJ, 2007.

[Gouin $\geq 2014$ ] H. Gouin, "Vesicles: a capillarity model revisited", in preparation.

[Gouin and Kosiński 1998] H. Gouin and W. Kosiński, "Boundary conditions for a capillary fluid in contact with a wall”, Arch. Mech. (Arch. Mech. Stos.) 50:5 (1998), 907-916.

[Helfrich 1973] W. Helfrich, "Elastic properties of lipid bilayers: theory and possible experiments", Z. Naturforsch. C 28 (1973), 693-703.

[Kobayashi and Nomizu 1963] S. Kobayashi and K. Nomizu, Foundations of differential geometry, vol. 1, Interscience, London, 1963.

[Levitch 1962] V. Levitch, Physicochemical hydrodynamics, Prentice-Hall, New Jersey, 1962.

[McBride et al. 2011] A. T. McBride, A. Javili, P. Steinmann, and S. Bargmann, "Geometrically nonlinear continuum thermomechanics with surface energies coupled to diffusion", J. Mech. Phys. Solids 59:10 (2011), 2116-2133.

[Noll and Virga 1990] W. Noll and E. G. Virga, "On edge interactions and surface tension", Arch. Rational Mech. Anal. 111:1 (1990), 1-31. 
[Ono and Kondo 1960] S. Ono and S. Kondo, "Molecular theory of surface tension in liquids", pp. 134-280 in Handbuch der Physik, Bd. X, Springer, Berlin, 1960.

[Rocard 1952] Y. Rocard, Thermodynamique, Masson, Paris, 1952.

[Rosen 2004] M. J. Rosen, Surfactants and interfacial phenomena, Wiley, New Jersey, 2004.

[Schwartz 1966] L. Schwartz, Théorie des distributions, Publications de l'Institut de Mathématique de l'Université de Strasbourg, No. IX-X. Nouvelle édition, entiérement corrigée, refondue et augmentée, Hermann, Paris, 1966.

[Seifert 1997] U. Seifert, "Configurations of fluid membranes and vesicles", Adv. Phys. 46 (1997), $13-137$.

[Serrin 1959] J. Serrin, "Mathematical principles of classical fluid mechanics", pp. 125-263 in Handbuch der Physik (herausgegeben von S. Flügge), Bd. 8/1, Strömungsmechanik I (Mitherausgeber C. Truesdell), Springer, Berlin, 1959.

[Slattery et al. 2007] J. C. Slattery, L. Sagis, and E.-S. Oh, Interfacial transport phenomena, Second ed., Springer, New York, 2007.

[Steigmann and Li 1995] D. J. Steigmann and D. Li, "Energy-minimizing states of capillary systems with bulk, surface, and line phases", IMA J. Appl. Math. 55:1 (1995), 1-17.

[Steinmann 2008] P. Steinmann, “On boundary potential energies in deformational and configurational mechanics”, J. Mech. Phys. Solids 56:3 (2008), 772-800.

[Toupin 1962] R. A. Toupin, "Elastic materials with couple-stresses", Arch. Rational Mech. Anal. 11 (1962), 385-414.

Received 5 Nov 2012. Revised 15 Mar 2013. Accepted 14 Apr 2013.

HENRI GOUIN: henri.gouin@univ-amu.fr

Aix-Marseille Université, CNRS, Centrale Marseille, M2P2 UMR 7340, 13451, Marseille, France 



\title{
PARTICLES FOR FLUIDS: SPH VERSUS VORTEX METHODS
}

\author{
Andrea Colagrossi, Giorgio Graziani And Mario Pulvirenti
}

We review the basic ideas underlying two popular particle methods for fluids: smoothed-particle hydrodynamics and the vortex method. We discuss convergence problems, numerical schemes, and examples of practical simulations.

\section{Introduction}

The Lagrangian form of the Navier-Stokes equations can be approximated with particle methods by replacing derivative operators through integral operators which are discretized on the particle locations. These particles carry the physical properties of a fluid dynamic system. Their trajectories, as well as the evolution of the transported quantities, are evaluated through the solution of ordinary differential equations. Particle methods such as vortex methods (VMs) and smoothed-particle hydrodynamics (SPH) present interesting features which allow the simulation of engineering problems involving complex interface dynamics such as vortex sheets and free-surface deformations (for a more general discussion of particle methods see, for example, [Koumoutsakos 2005]). SPH is a particle method introduced by Lucy [1977] and further developed by Monaghan (see, for example, [Monaghan 1992]) to study self-gravitating fluids. The basic idea of the method is to consider the fluid as an ensemble of (smooth) particles whose evolution simulates that of the fluid under consideration. Each particle has a form factor (a symmetric, regular, nonnegative function centered on the particle position) which simulates the mass distribution around the center of each particle. Moreover the particles move in the force field generated by the whole particle system.

For many years SPH was used for astronomical problems; later, it was applied to several physics and engineering problems, ranging from solid mechanics to multiphase flows. Monaghan [1994] first applied it for simulating free-surface inviscid flows. Compared to the Eulerian finite difference method, SPH can handle breaking and fragmentation phenomena in a more natural way, due to its Lagrangian nature.

MSC2010: primary 35Q30, 35Q35, 76D17, 35Q31; secondary 35L60, 35Q70.

Keywords: particle methods, smoothed-particle hydrodynamics, vortex method, convergence, viscous flows. 
Thanks to this valuable feature, interest in SPH has grown quickly in the last decade and several researchers have devoted their efforts to improving this method.

Clearly the basic requirement for this particle method is its convergence to the right solution of the fluid dynamic equations when the number of particles diverges. In this paper we want to discuss this issue in connection with Vlasov dynamics, which is strictly related to problem at hand.

Another well-known method discussed in the present paper is the vortex method (VM), which works for two-dimensional incompressible flows. This is also a particle approximation of the incompressible Euler and Navier-Stokes equations in the vorticity formalism. It is conceptually related to the SPH method because both approximate the fluid equations in a suitable mean-field limit. Here we want to discuss these methods exploiting this aspect.

As we shall discuss in some detail in the sequel, SPH and the VM seem to be effective in approaching practical problems. In this work numerical solutions of viscous flows obtained with both methods are discussed showing the benefits and drawbacks of the two solvers. In particular, using the same spatial resolution, the VM provides numerical solutions with higher accuracy and lower CPU costs than does SPH. However, it is important to underline that the latter method can be applied much more easily on a wider range of engineering problems (free-surface, multiphase, interfaces, compressible flows, etc.), while for the VM even extension from two to three-dimensional framework is not straightforward. Therefore, the choice between the two particle methods is still strongly problem dependent. Another important point regards the parallelization of the different algorithms. Since the SPH model is based on short-range interactions, it is quite easy to parallelize in a very efficient way (see, for example, [Marrone et al. 2012]). This is not the case for the VM, where the evaluation of velocity fields requires more effort to parallelize (see, for example, [Barnes and Hut 1986; Antonuccio-Delogu et al. 1996]).

The plan of the paper is as follows. In Section 2 we introduce SPH and the VM as regards their general structure and the convergence problems. In Section 3 we discuss some numerical schemes suited for practical implementations of the two methods. Finally, Section 4 is devoted to examples of practical simulations.

\section{Convergence of particle methods}

2.1. Euler equation. In the present subsection we introduce the basic equations we want to investigate. Let us start with the isentropic Euler equation for a pressure law $p=(1 /(\alpha+2)) \rho^{\alpha+2}$, where $p$ is the pressure and $\rho$ is the density:

$$
\begin{cases}\frac{\partial \rho}{\partial t}+\nabla \cdot(\rho u)=0, & \frac{\partial u}{\partial t}+(u \cdot \nabla u)+\rho^{\alpha} \nabla \rho=0, \\ \rho(x, 0)=\rho_{0}(x), & u(x, 0)=u_{0}(x) .\end{cases}
$$


Here $u=u(x, t)$ denotes the velocity field, $x \in \mathbb{R}^{d}, d=2,3$, the dimension of the physical space, and $t \in \mathbb{R}$ the time.

The incompressible Euler equation reads

$$
\frac{\partial u}{\partial t}+(u \cdot \nabla u)+\rho_{0}^{-1} \nabla p=0, \quad \nabla \cdot u=0, \quad u(x, 0)=u_{0}(x) .
$$

Here we are considering the fluid invading the whole space. However most of the applications we are interested in the fluids are confined in domains with boundaries, in which also viscous effects are not negligible. We discuss these important aspects later on.

2.2. The Vlasovequation. When dealing with large weakly interacting particle systems in stellar dynamics or in the physics of plasmas, we often use the following evolution equation for $f(x, v, t)$, the fraction of particles in the cell of the phase space around $(x, v)$ of size $d x d v$ (or the probability density of finding a given particle in $(x, v))$ :

$$
\left(\partial_{t}+v \cdot \nabla_{x}+F \cdot \nabla_{v}\right) f(x, v, t)=0 .
$$

Here $x \in \mathbb{R}^{d}$ denotes the position and $v \in \mathbb{R}^{d}$ the velocity of a test particle, while $t \in \mathbb{R}$ denotes the time, with $d=2,3$ the dimension of the physical space.

Note that (2-3) is the usual Liouville equation for a single particle in a force field $F=F(x, t)$, so that (2-3) is solved by the formula

$$
f(x, v, t)=f_{0}\left(\Psi^{-t}(x, v)\right),
$$

or, equivalently,

$$
\int d x d v f(x, v, t) g(x, v)=\int d x d v f_{0}(x, v) g\left(\Psi^{t}(x, v)\right),
$$

where $f_{0}$ is the initial datum for $(2-3), \Psi^{t}(x, v)=(x(t), v(t))$ is the flow solution to the ordinary differential system

$$
\dot{x}(t)=v(t), \quad \dot{v}(t)=F(x(t), t),
$$

with initial datum $\Psi^{0}(x, v)=(x, v)$, and $g$ is any bounded continuous function.

Equation (2-3) differs from the Liouville equation because $F$ is not known a priori, but depends on the solution itself, via the self-consistent formula

$$
F(x, t)=\int d x K(x-y) \rho(y, t) d y,
$$

where

$$
\rho(x, t)=\int d v f(x, v, t)
$$

is the spatial density and $K: \mathbb{R}^{d} \rightarrow \mathbb{R}^{d}$ is a given kernel. In other words (2-3) is a 
nonlinear equation because the vector field $F$ depends on the solution $f$.

Clearly the interesting case is when the force kernel $K$ arises from a potential, namely

$$
K(x)=-\nabla \varphi(x) .
$$

If $\varphi$ is assumed to be smooth, it is possible to show that there exists a unique solution to the initial value problem associated with (2-3).

Following [Dobrušin 1979] (see also [Braun and Hepp 1977; Neunzert 1984]), we can establish the following result:

Theorem 1. Consider the N-particle evolution

$$
\left\{\begin{array}{l}
\dot{x}_{i}(t)=v_{i}(t), \\
\dot{v}_{i}(t)=-\frac{1}{N} \sum_{\substack{1 \leq j \leq N \\
j \neq i}} \nabla \varphi\left(x_{i}(t)-x_{j}(t)\right), \\
x_{i}(0)=x_{i}, \quad v_{i}(0)=v_{i},
\end{array}\right.
$$

where $i=1, \ldots, N$. Introduce an empirical measure defined on the one-particle phase space by

Then if

$$
\mu_{N}(d x, d v ; t)=\frac{1}{N} \sum_{i=1}^{N} \delta\left(x-x_{i}(t)\right) \delta\left(v-v_{i}(t)\right) d x d v
$$

$$
\lim _{N \rightarrow \infty} \int d \mu_{N}(x, v ; 0) g(x, v)=\int f_{0}(x, v) g(x, v) d x d v,
$$

for all functions $g$ continuous and bounded, then, for all $t>0$,

$$
\lim _{N \rightarrow \infty} \int d \mu_{N}(x, v ; t) g(x, v)=\int f(x, v, t) g(x, v) d x d v,
$$

where $f(t)$ solves (2-3) with initial datum $f_{0}$.

We do not present the proof of this theorem, but some comments are in order. Let $M$ be the space of the Borel probability measure on $\mathbb{R}^{d}$. We first observe that there exists a unique measure-valued solution $t \rightarrow v(t) \in \mathcal{M}$ to (2-3), in the following weak form (which does not require any smoothness of $v$ ), for any smooth test function $g$ :

$$
\frac{d}{d t}\langle v, g\rangle=\left\langle\nu, v \cdot \nabla_{x} g\right\rangle+\left\langle v, F \cdot \nabla_{v} g\right\rangle,
$$

where

$$
\langle v, g\rangle=\int d x \int d v g(x, v) v(d x, d v),
$$

and $F=K * v$ is the self-consistent force.

Using integration by parts, it is easy to show that (2-13) holds whenever $f(t)$ is a classical solution to (2-3) and $v(d x, d v, t)=f(t) d x d v$. 
Moreover a trivial computation also shows that $\mu_{N}$ is a weak solution to (2-13). Therefore Theorem 1 says precisely that the solution to the Vlasov equation at any fixed time $t>0$, is continuous with respect to the initial data, for the topology induced by the weak convergence of the measures, expressed by condition (2-11).

There is a fruitful connection between Vlasov dynamics and the solutions of the problems given by (2-1) and (2-2), which we shall discuss in the next sections.

2.3. The SPH model. Let us start to consider the Euler problem (2-1) for the particular case $\alpha=0$ :

$$
\left\{\begin{array}{l}
\rho_{t}+\nabla \cdot(\rho u)=0, \\
u_{t}+(u \cdot \nabla u)+\nabla \rho=0, \\
\rho(x, 0)=\rho_{0}(x), \quad u(x, 0)=u_{0}(x) .
\end{array}\right.
$$

In Lagrangian form it reads

$$
\left\{\begin{array}{l}
\ddot{\Phi}_{t}(x)=-\nabla \rho\left(\Phi_{t}(x), t\right), \\
\int \rho(x, t) g(x)=\int \rho_{0}(x) g\left(\Phi_{t}(x)\right), \\
\Phi_{0}(x)=x, \quad \dot{\Phi}_{0}(x)=u_{0}(x),
\end{array}\right.
$$

where $g$ is a bounded continuous function and $\Phi_{t}: \mathbb{R}^{d} \times \mathbb{R}^{+} \rightarrow \mathbb{R}^{d}$ is the solution flow.

The Eulerian velocity is recovered via the formula

$$
u\left(\Phi_{t}(x), t\right)=\dot{\Phi}_{t}(x)
$$

Recall that a solution to the Lagrangian problem (2-15) (also in Eulerian form, (2-14)) exists only locally in time; see, for example, [Majda 1984].

Next we regularize the problem by setting

$$
\left\{\begin{array}{l}
\ddot{\Phi}_{t}(x)=-\nabla\left(\delta_{\varepsilon} * \rho\right)\left(\Phi_{t}(x), t\right), \\
\int \rho(x, t) g(x)=\int \rho_{0}(x) g\left(\Phi_{t}(x)\right), \\
\Phi_{0}(x)=x, \quad \dot{\Phi}_{0}(x)=u_{0}(x),
\end{array}\right.
$$

where the form factor $\delta_{\varepsilon}$ is a positive, smooth approximation of the $\delta$ function. Here $\varepsilon$ is a small positive parameter such that

$$
\delta_{\varepsilon} \rightarrow \delta
$$

in the sense of the weak convergence of the measures (2-11), as $\varepsilon \rightarrow 0$.

We note that, as we shall see in a moment, problem (2-16) has a global solution (even for initial densities $\rho_{0}$ which are measures). 
The connection with the Vlasov equation discussed in the last section is clear. Indeed consider the solution flow (2-6) to the Vlasov equation with

$$
F=K * \rho, \quad K=-\nabla \delta_{\varepsilon},
$$

and with initial datum

$$
f_{0}(x, v) d x d v=\rho_{0}(x) \delta\left(v-u_{0}(x)\right) d x d v .
$$

Then, setting

$$
\left(\Phi_{t}(x), \dot{\Phi}_{t}(x)\right)=\Psi^{t}\left(x, u_{0}(x)\right)
$$

the time evolved measure $f(x, v, t) d x d v$ satisfies

$$
\begin{aligned}
\int f(x, v, t) g(x, v) d x d v & =\int f_{0}(x, v) g\left(\Psi^{t}(x, v)\right) d x d v \\
& =\int d x d v \rho_{0}(x) \delta(v-u(x)) g\left(\Psi^{t}(x, v)\right) \\
& =\int d x d v \rho_{0}(x) g\left(\Phi_{t}(x), \dot{\Phi}_{t}(x)\right),
\end{aligned}
$$

which implies (2-16). In fact, if $g$ depends only on $x$, we get the second formula of (2-16), while the first one follows by the obvious remark that $\ddot{\Psi}_{t}(x, u(x))=\ddot{\Phi}_{t}(x)$. In other words, the solution to the problem (2-16) is a particular solution to the Vlasov equation for the special initial datum (2-17). Note that although such a solution, due to the smoothness of $\delta_{\varepsilon}$, does exist for any arbitrary time $t$, it not necessarily take the form

$$
f(x, v) d x d v=\rho(x, t) \delta(v-u(x, t)),
$$

where the velocity and the density fields $u(x, t)$ and $\rho(x, t)$ satisfies the equivalent of (2-14) with the corresponding regularized pressure field. Actually, by using the well-known equivalence between the Lagrangian and the (regularized) Eulerian pictures, this is guaranteed only locally in time, namely in the existence time interval of a smooth solution to the regularized Euler equations.

The SPH model in the present context is the $N$-particle system satisfying

$$
\left\{\begin{array}{l}
\dot{x}_{i}(t)=v_{i}(t), \\
\dot{v}_{i}(t)=-\frac{1}{N} \sum_{\substack{1 \leq j \leq N \\
j \neq i}} \nabla \delta_{\varepsilon}\left(x_{i}(t)-x_{j}(t)\right), \\
x_{i}(0)=x_{i}, \quad v_{i}(0)=u_{0}\left(x_{i}\right),
\end{array}\right.
$$

for $i=1, \ldots, N$, and

$$
\mu_{N}(d x, t)=\sum_{i=1}^{N} \delta\left(x-x_{i}(t)\right) d x .
$$


By Theorem 1, we have

$$
\mu_{N}(d x, t) \rightarrow \rho(x, t)
$$

weakly, as $N \rightarrow \infty$ for a fixed $\varepsilon$, where $\rho$ solves (2-16).

We now analyze the general case including $\alpha \neq 0$. The Lagrangian form is

$$
\left\{\begin{array}{l}
\ddot{\Phi}_{t}(x)=-\rho^{\alpha} \nabla \rho\left(\Phi_{t}(x), t\right), \\
\int \rho(x, t) g(x)=\int \rho_{0}(x) g\left(\Phi_{t}(x)\right), \\
\Phi_{0}(x)=x, \quad \dot{\Phi}_{0}(x)=u_{0}(x),
\end{array}\right.
$$

while its regularized version is

$$
\left\{\begin{array}{l}
\ddot{\Phi}_{t}(x)=-\left(\delta_{\varepsilon} * \rho\right)^{\alpha} \nabla\left(\delta_{\varepsilon} * \rho\right)\left(\Phi_{t}(x), t\right), \\
\int \rho(x, t) g(x)=\int \rho_{0}(x) g\left(\Phi_{t}(x)\right), \\
\Phi_{0}(x)=x, \quad \dot{\Phi}_{0}(x)=u_{0}(x) .
\end{array}\right.
$$

The SPH method can be suitably modified to give

$$
\left\{\begin{array}{l}
\dot{x}_{i}(t)=v_{i}(t), \\
\dot{v}_{i}(t)=-\frac{1}{N} \sum_{\substack{1 \leq j \leq N \\
j \neq i}}\left(\frac{1}{N} \sum_{\substack{1 \leq k \leq N \\
k \neq i}} \delta_{\varepsilon}\left(x_{i}(t)-x_{k}(t)\right)\right)^{\alpha} \nabla \delta_{\varepsilon}\left(x_{i}(t)-x_{j}(t)\right), \\
x_{i}(0)=x_{i}, \quad v_{i}(0)=u_{0}\left(x_{i}\right),
\end{array}\right.
$$

where $i=1, \ldots, N$.

It is possible to adapt the techniques we have discussed so far for the case $\alpha=$ 0 to the present case. All the convergence results we have discussed so far are described in detail in [Mas-Gallic and Raviart 1987; Oelschläger 1991; Di Lisio 1995; Di Lisio et al. 1997; 1998; Ben Moussa and Vila 2000; Ben Moussa 2006].

Actually the SPH models we have introduced approximate problem (2-25) which, in Eulerian form, reads

$$
\left\{\begin{array}{l}
\partial \rho / \partial t+\nabla \cdot(\rho u)=0, \\
\partial u / \partial t+(u \cdot \nabla u)+\left(\rho * \delta_{\varepsilon}\right)^{\alpha} \nabla\left(\rho * \delta_{\varepsilon}\right)=0, \\
\rho(x, 0)=\rho_{0}(x), \quad u(x, 0)=u_{0}(x)
\end{array}\right.
$$

The last step is to show that the solution of (2-27) converges to that of (2-1) when the parameter $\varepsilon$ vanishes. This is It is rather technical, but it can be done; see [Di Lisio et al. 1997] for details.

2.4. The vortex model. Let us consider the Euler equation (2-2) in the plane. Define the vorticity $\omega=\omega(x, t)=\partial_{x_{1}} u_{2}-\partial_{x_{2}} u_{2}$ (which is a scalar quantity in the 
present context). Then we obtain

$$
\left(\partial_{t}+u \cdot \nabla\right) \omega(x, t)=0 .
$$

Here $x=\left(x_{1}, x_{2}\right) \in \mathbb{R}^{2}, t \in \mathbb{R}^{+}$, and $u=u(x, t) \in \mathbb{R}^{2}$ is the velocity field. Due to the incompressibility condition $\nabla \cdot u=0$, we can reconstruct the velocity field $u$ from the vorticity profile $\omega$. The result is

$$
u=\nabla^{\perp} \psi, \quad \psi=-\Delta^{-1} \omega .
$$

Explicitly:

$$
u=K * \omega, \quad K(x)=\nabla^{\perp} g(x)=-\frac{1}{2 \pi} \frac{x^{\perp}}{|x|^{2}},
$$

where

$$
g(x)=-\frac{1}{2 \pi} \log |x|
$$

is the fundamental solution for the Poisson equation in the plane.

There is an evident similarity between (2-28) and the Vlasov equation (2-3) which suggests the following particle model:

$$
\dot{x}_{i}(t)=\frac{1}{N} \sum_{\substack{1 \leq j \leq N \\ j \neq i}} K\left(x_{i}(t)-x_{j}(t)\right), \quad x_{i}(0)=x_{i}, \quad i=1, \ldots, N .
$$

A preliminary difficulty in dealing with (2-31) is the singularity of $K(x)$ for $x$ close to 0 . Regularizing the divergence at the origin via a smooth kernel $K_{\varepsilon}$, we substitute (2-31) by

$$
\dot{x}_{i}(t)=\frac{1}{N} \sum_{\substack{1 \leq j \leq N \\ j \neq i}} K_{\varepsilon}\left(x_{i}(t)-x_{j}(t)\right), \quad x_{i}(0)=x_{i}, \quad i=1, \ldots, N .
$$

Theorem 1 allows us to show that the empirical measure

$$
\mu_{N}(d x ; t)=\sum_{i=1}^{N} \delta\left(x-x_{i}(t)\right) d x
$$

is close to $\omega_{\varepsilon}$ which solves

$$
\left(\partial_{t}+u_{\varepsilon} \cdot \nabla\right) \omega_{\varepsilon}(x, t)=0,
$$

where

$$
u_{\varepsilon}=K_{\varepsilon} * \omega_{\varepsilon} .
$$

As before the last step is to show that $\omega_{\varepsilon} \rightarrow \omega$ where $\omega$ solves (2-28).

In the present context we are considering $\omega$ as a probability measure, however the generalization to arbitrary signed measure is straightforward. 
All these steps can be performed, as discussed in [Marchioro and Pulvirenti 1982; 1984; 1994].

The literature on the vortex model is huge and it would be hopeless to cover it exhaustively. We mention only that the vortex system has been largely investigated from the point of view of the general theory of the Hamiltonian dynamical system, see, for instance, [Newton 2001].

When the fluid is confined in domain with a boundary, say $\Omega$, the VM can be easily extended to describe this situation. It is enough to replace $K$ with $K_{\Omega}$, where

$$
K_{\Omega}(x)=\nabla^{\perp} g_{\Omega}(x),
$$

with $g_{\Omega}$ being the fundamental solution of the Poisson equation in the domain $\Omega$, with the boundary conditions constantly zero.

Note that the usual impermeability condition for the incompressible Euler flow, namely

$$
u(x, t) \cdot n=0 \quad \text { on } \partial \Omega,
$$

where $n$ is the normal to the surface $\partial \Omega$, is automatically satisfied (see, for example, [Marchioro and Pulvirenti 1994]).

Moreover, when the viscous effects are not negligible, we have to consider the Navier-Stokes equation, which, in terms of vorticity, reads

$$
\left(\partial_{t}+u \cdot \nabla\right) \omega(x, t)=v \nabla^{2} \omega \text { for all } x \in \Omega .
$$

Here $v>0$ denotes the kinematic viscosity coefficient.

When $\Omega=\mathbb{R}^{2}$ the viscosity effect can be included in the VMs just by adding $N$ independent Brownian motions to (2-31). This idea goes back to Chorin [1973]. A mathematical analysis of this approach can be found in [Marchioro and Pulvirenti 1982; 1984].

However the vorticity formalism for the Navier-Stokes equation, while convenient as regards the possibility of a particle approximation in terms of stochastic particle systems or in terms of other methods (see the numerical scheme implemented later on), exhibits subtle difficulties in describing the boundary conditions. Indeed the no-slip condition $u=0$ on the boundary is not easily read as a simple condition on the behavior of the vorticity field $\omega$ on the boundary. As we said we have automatically $u(x, t) \cdot n=0$, but we have also to ensure that the tangential component of the velocity must vanish.

Again going back to Chorin [1973], this problem can be overcome by assuming the Neumann boundary condition for the Laplace operator appearing on the righthand side of (2-36). This preserves the total amount of vorticity. Next we can restore the correct boundary conditions by putting a (singular) source of vorticity 
on the boundary, computed in such a way that the tangential component of the velocity on the boundary vanishes.

A rigorous mathematical analysis of this procedure can be found in [Benfatto and Pulvirenti 1984; Boldrighini and Buttà 2011].

From a practical view point a numerical scheme can be implemented by the use of the so-called Chorin-Marsden product formula, which consists in the following procedure. First we evolve the flow by the Euler equation, for a very small time $h$. Then we restore the correct boundary conditions by adding a vortex sheet to compensate for the tangential part of the velocity produced by the first step. Finally we evolve the system by the heat flow with Neumann boundary conditions for a small time $h$. The convergence of this method has been proved in [Benfatto and Pulvirenti 1986] in the case of the half-plane.

This class of ideas has been used for the numerical simulations presented in the sequel.

\section{Description of numerical schemes for SPH and VMs}

In the present section the numerical schemes for SPH and the VM are described. Different formulations are available in the literature; here two are briefly recalled in the context of a viscous Newtonian fluid. Further details can be found in [Graziani et al. 1995; Federico et al. 2012].

3.1. Smoothed-particle hydrodynamics formulation. In this section a SPH scheme is briefly described. The fluid is assumed to be barotropic and weakly compressible and the reference equations are the Navier-Stokes equations. The flow domain $\Omega$ is bounded by the surface $\partial \Omega_{b}$ of the solid body and by the lateral walls, where a simple free-slip condition is enforced. The lateral walls are far enough from the body to avoid significant effects on it. No-slip boundary conditions are enforced on the body surface.

As discussed in [Molteni et al. 2007; Antuono et al. 2010] different state equations, $p=f(\rho)$, can be used in the SPH scheme to model weakly compressible fluids. However, as shown below, only with the choice $\alpha=0$ it is possible to link (2-22) with the equation used in the classical SPH models. Indeed, (2-26) does not preserve the momentum conservation of the particle system, leading to a less robust and less stable numerical scheme. Therefore, here, a simple quadratic state equation (that is, $\alpha=0$ according to Section 2.1) is used to match the pressure and density field:

$$
p=A \rho^{2}+B=\frac{c_{0}^{2}}{2 \rho_{0}}\left(\rho^{2}-\rho_{0}^{2}\right)+p_{0} .
$$

Here, $c_{0}, \rho_{0}$, and $p_{0}$ are the speed of sound, the density, and the pressure with 
the fluid at rest, respectively. It is important to underline that the constant $B=$ $\left(p_{0}-c_{0}^{2} \rho_{0} / 2\right)$ is used for the purpose of numerical stability to avoid the region where the so-called tensile instability can develop [Swegle et al. 1995]. The speed of sound, $c_{0}$, is set in order to guarantee density variation smaller than $0.01 \rho_{0}$, or, in other words, the Mach number, Ma, of the simulation is less than 0.1. Indeed, as shown in [Majda 1984], in the limit for Ma going to zero the solution of the isentropic Euler equation (2-1) converges to the incompressible one (2-2).

Given a set of particles each characterized by its own mass $m_{i}$, the particle densities can be expressed through the distribution (2-23). In contrast with the method as presented in Section 2, we find it convenient to choose different masses $m_{i}$ in place of identical $m_{i}=1 / N$; therefore (2-23) becomes

$$
\rho_{i}=\sum_{j} \delta_{\epsilon}\left(x_{i}-x_{j}\right) m_{j}
$$

where $\delta_{\epsilon}=\delta_{\epsilon}\left(x_{j}-x_{i}\right)$ is the kernel function. In this work a renormalized Gaussian kernel (see, for example, [Molteni and Colagrossi 2009]) has been used. $\delta_{\epsilon}$ has a compact support of radius $3 \epsilon$, where $\epsilon$ is the smoothing length.

Deriving in time (3-2) we get the continuity equation in the SPH formalism:

$$
\dot{\rho}_{i}=\sum_{j}\left(u_{i}-u_{j}\right) \cdot \nabla_{i} \delta_{\epsilon}\left(x_{i}-x_{j}\right) m_{j} .
$$

The symbol $\nabla_{i}$ indicates differentiation with respect to the position of the $i$-th particle; for the sake of simplicity in what follows $\nabla_{i} \delta_{\epsilon}\left(x_{i}-x_{j}\right)$ will be indicated just with $\nabla_{i} \delta_{\epsilon}$. From the numerical point of view, the evaluation of the density through time integration of (3-3) is preferable with respect to the direct use of (3-2) and becomes crucial when dealing with free-surface flows (see, for example, [Colagrossi et al. 2009]).

In this section we make use of useful new relations, consequences of the energy conservation. Indeed, for an isentropic fluid the sum of the kinetic energy $\mathscr{E}_{k}$ and the internal energy $\mathscr{E}_{i}$ of the particles system is conserved:

$$
\dot{\mathscr{E}}_{k}+\dot{\mathscr{E}}_{i}=\sum_{i} m_{i} u_{i} \dot{u}_{i}+\sum_{i} m_{i} \frac{p_{i}}{\rho_{i}^{2}} \dot{\rho}_{i}=0
$$

By substituting (3-3) in (3-4) and using the kernel property

$$
\nabla_{i} \delta_{\epsilon}=-\nabla_{j} \delta_{\epsilon}
$$

the acceleration of the $i$-th particle is given by

$$
\dot{u}_{i}=-\sum_{j}\left(\frac{p_{i}}{\rho_{i}^{2}}+\frac{p_{j}}{\rho_{j}^{2}}\right) \nabla_{i} \delta_{\epsilon} m_{j} .
$$


When we use this equation for $\dot{\rho}_{i}$ and $\dot{u}_{i}$, the SPH scheme reads

$$
\left\{\begin{array}{l}
\dot{\rho}_{i}(t)=-\sum_{j}\left(u_{j}-u_{i}\right) \cdot \nabla_{i} \delta_{\epsilon} m_{j}, \\
\dot{u}_{i}(t)=-\sum_{j}\left(\frac{p_{i}}{\rho_{i}^{2}}+\frac{p_{j}}{\rho_{j}^{2}}\right) \nabla_{i} \delta_{\epsilon} m_{j}+\frac{v}{\rho_{0}} \sum_{j} \pi_{i j} \nabla_{i} \delta_{\epsilon} m_{j}, \\
\dot{x}_{i}(t)=u_{i}(t),
\end{array}\right.
$$

where $\rho_{i}, p_{i}, u_{i}$, and $m_{i}$ are, respectively, the density, pressure, velocity, and mass of the $i$-th particle, while $v$ denotes the fluid kinematic viscosity.

Thanks to the use of (3-4) the exact momentum conservation in (3-6) is guaranteed regardless of the state equation adopted. Furthermore, setting $v=0, m_{j}=1 / N$, and $p=\rho^{2} / 2$ in (3-6), then (2-22), discussed in Section 2.3, is recovered.

The viscous forces are modeled through the viscous formula of [Monaghan and Gingold 1983], which preserves both linear and angular momenta:

$$
\pi_{i j}=2(n+2) \frac{\left(u_{j}-u_{i}\right) \cdot\left(x_{j}-x_{i}\right)}{\left\|x_{j}-x_{i}\right\|^{2}},
$$

where $n$ is the spatial dimension of the problem at hand. For $\epsilon \rightarrow 0$ the system (3-6) converges to the Navier-Stokes equations (see, for example, [Colagrossi et al. 2011]).

The fluid particles are initially positioned using the algorithm described in [Colagrossi et al. 2012]. Thanks to this procedure, at the initial instant all particles have approximately the same volume, namely $V_{0}$, which is equal to the fluid domain volume divided by the number of fluid particles. Consistently, the particle mean spacing is denoted by $\Delta x=V_{0}^{1 / n}$. The average number of particles in the kernel support is set by choosing the ratio $\epsilon / \Delta x$. In the present work $\epsilon / \Delta x$ is set equal to 1.33 , which in two dimensions corresponds to about 50 interacting particles.

Along with the volume distribution, the initial pressure and the velocity fields are prescribed as well. The initial density distribution $\rho_{i}\left(t_{0}\right)$ is evaluated by means of the state equation and the particle masses are computed through the equation $m_{i}=V_{0} \rho_{i}\left(t_{0}\right)$. The mass of the $i$-th particle remains constant during the time evolution ensuring the total mass conservation of the particle system.

\subsubsection{Enforcement of the solid-boundary condition through a ghost-fluid method.} In the present work the ghost-fluid technique is used to enforce proper boundary conditions on the body surface. Specifically, the solid domain is modeled through a set of "imaginary particles" (hereinafter denoted as ghost particles and labeled with the subscript $s$ ) and all the fluid fields (that is, velocity and pressure) are extended on these fictitious particles through proper mirroring techniques. Different mirroring techniques are adopted to enforce different boundary conditions (for example, Dirichlet or Neumann conditions). To this end, the solid surface is 
discretized in equispaced body nodes and a layer of ghost particles is disposed in the solid region. The ghost particle positions have been obtained by using the technique described in [Marrone et al. 2011]. The pressure and velocity energy assigned to the fixed ghost particles, namely $\left(u_{s}, p_{s}\right)$, are computed by using the values obtained at specific interpolation nodes internal to the fluid and uniquely associated with the fixed ghost particles. Hereinafter, the interpolated values are indicated through $\left(u^{*}, p^{*}\right)$.

The pressure field $p_{s}$ is mirrored on the fixed ghost particles to enforce the Neumann condition

$$
\frac{\partial p}{\partial n}=\rho\left(f \cdot n-\dot{u}_{b} \cdot n\right)
$$

where $f$ is a generic body force and $u_{b}$ is the velocity of the solid boundary (for details see [Marrone et al. 2011]). This leads to

$$
p_{s}=p^{*}+\frac{\partial p}{\partial n} \cdot\left(x^{*}-x_{s}\right) .
$$

The velocity field is the object of a specific treatment. The ghost velocity $u_{s}$ depends on both $u^{*}$ and $u_{b}$, the latter being the velocity of the nearest body node. De Leffe et al. [2011] found that different mirroring techniques have to be used to evaluate $\langle\nabla \cdot u\rangle$ and $\left\langle\nabla^{2} u\right\rangle$ and to avoid inconsistencies and loss of accuracy. The specific mirroring techniques treat differently the components of $u^{*}$ in the normal and tangential directions to the solid surface. The velocity-divergence operator is convergent and consistent if the normal component of $u^{*}$ is mirrored in the frame of reference of the solid profile (see [Colagrossi and Landrini 2003]), leaving the tangential component unaltered. Conversely, to evaluate $\left\langle\nabla^{2} u\right\rangle$, the velocity field has to be mirrored to approximate no-slip conditions along the solid bodies. A common way is to reverse the tangential component with a linear extrapolation, leaving the normal one unaltered. The consistency of this second mirroring procedure has been discussed in [Macià et al. 2011].

Using the momentum exchange between fluid and ghost particles it is possible to evaluate the global loads acting on the solid bodies:

$$
\boldsymbol{F}_{\text {fluid-solid }}=\sum_{i \in \text { fluid }} \sum_{j \in \text { solid }}\left(-m_{i}\left(\frac{p_{j}}{\rho_{j}^{2}}+\frac{p_{i}}{\rho_{i}^{2}}\right)+v \frac{m_{i}}{\rho_{0}} \pi_{i j}\right) \nabla_{i} \delta_{\epsilon} m_{j},
$$

in which the first term on the right-hand side represents the pressure component and the second acts as the viscous component of the stress tensor.

3.2. Viscous VM with operator splitting technique. In order to analyze the vortex shedding from the body, the mathematical model can be equivalently recast in terms of the vorticity transport equation. This approach allows for removing the 
pressure from the unknown variables and for the implicit enforcement of the farfield boundary conditions.

In the case of a two-dimensional flow, the governing equation is written as

$$
\frac{D \omega}{D t}=\left(\partial_{t}+u \cdot \nabla\right) \omega(x, t)=v \nabla^{2} \omega \text { for all } x \in \Omega,
$$

where $D / D t$ denotes the material derivative.

Let us now briefly describe some detail of the numerical procedure for the solution of the governing equations. The governing equation for the vorticity field (3-10) accounts for the simultaneous advection and diffusion of this quantity. According to the well-known operator splitting approach [Chorin 1973], these two steps are separately accounted for in the computational procedure. The approximate solution of the governing equation is obtained by the sequential solution of the diffusive "Stokes" step followed by an inviscid "Euler" step for the vorticity advection. The Stokes step represents the pure diffusion of the vorticity:

$$
\frac{\partial \omega}{\partial t}=v \nabla^{2} \omega
$$

in the absence of motion combined with a vorticity generation on the solid boundary to restore the no-slip boundary condition. Indeed, the presence of a body within the flow field, requires to model the vorticity generation process on the solid walls which allows for the vortex shedding effects. Therefore, vorticity is generated on the body boundary to enforce the no-slip condition.

In the Euler step an inviscid fluid is considered where the vorticity of each particle is conserved along its Lagrangian motion:

$$
\frac{D \omega}{D t}=0 .
$$

After the splitting of the governing equation, it is clear that an appropriate procedure both for the diffusion and for the advection of the vorticity must be devised. The natural choice for the numerical solution scheme appears now as a viscous $V M$ which is based on the original papers [Chorin 1973; 1978] together with a deterministic diffusion algorithm [Benson et al. 1989] (see [Graziani et al. 1995] for more details about the adopted computational procedure).

3.2.1. Particle discretization of the vorticity field. The VM discretizes the vorticity field $\omega(x, t)$ and replaces it with a set of $N_{\text {vor }}$ point vortices with circulation $\Gamma\left(x_{j}, t\right)$ (which we write as $\Gamma_{j}(t)$ below):

$$
\omega(x, t)=\sum_{j=1}^{N_{\text {vor }}} \Gamma_{j}(t) \delta_{\epsilon}\left(x-x_{j}\right),
$$


where the spatial distribution has been regularized as already mentioned in the second section. The evolution of the vorticity field is now computed through the advection and the diffusion of the point vortices. The adoption of the vorticity as the dependent variable avoids the discretization of the irrotational regions while allowing for reserving the computational resources to the vortical zones. This is a major difference with respect to other commonly used computational schemes which require the discretization of the whole solution domain (as for the SPH method described above). At the same time, the adoption of an integral representation for the velocity field yields the exact enforcement of the far-field boundary conditions.

One of the drawbacks of the purely Lagrangian VMs stems from the excessive clustering or rarefaction of the vortex particles which requires a, somewhat nonphysical, redistribution procedure. In our case the adopted diffusion scheme avoids this possibility because a computational grid is used, only for this step, and each particle gives its diffusive contribution to the circulation of the new particles located on regular mesh nodes. At the end of the Stokes step the former set of vortex particles is replaced by that defined in the nodes of the computational mesh.

Therefore, the present hybrid Eulerian-Lagrangian computational scheme allows for the convenient evaluation of the particles' advection as well as for the efficient redistribution of the vorticity during diffusion.

We briefly recall the essential details of the four involved substeps: integral representation of the velocity field, vorticity generation, diffusion, and advection.

(1) Integral representation of the velocity field. By starting from the Helmholtz decomposition of the velocity field and considering the incompressibility constraint and the impermeability condition on $\partial \Omega$, the velocity field can be expressed by

$$
u\left(x^{\star}\right)=u_{\infty}+\int_{\partial \Omega} u_{\tau}(x) K\left(x, x^{\star}\right) d l+\int_{\Omega} \omega(x) K\left(x, x^{\star}\right) d S,
$$

where the operator $K$ has been defined in Section 2. Equation (3-14) is a way to represent the domain-dependent Green's function.

The unknown tangential velocity component $u_{\tau}$ appearing in (3-14) must be evaluated by solving an integral equation obtained by locating $x^{\star}$ on the body surface and discretizing it on $\partial \Omega$ :

$$
\begin{aligned}
& \frac{u_{i \tau}}{2}=u_{\infty \tau}+\sum_{k} u_{k \tau} \int_{\partial \Omega_{k}} \frac{\partial G}{\partial n}\left(x_{k}, x_{i}\right) d l_{k}+\tau_{i} \cdot \sum_{j} \Gamma_{j} K\left(x_{j}, x_{i}\right) d S_{j} \\
& \text { for all } x_{i} \in \partial \Omega \text {. }
\end{aligned}
$$

The last term in (3-15) is the tangential velocity on the body surface induced by the vortices. In order to evaluate it, the multipole expansion for the kernel $K$ is adopted with reduced computational cost (see [Van Dommelen and Rundensteiner 
1989]). The numerical solution of (3-15) requires a specific algorithm; details can be found in, for example, [Graziani and Landrini 1999].

At this point the enforcement of the no-slip boundary condition can be taken into account through the next step.

(2) Vorticity generation on the solid body profile. In order to satisfy the no-slip boundary condition, the adopted operator splitting approach must be complemented, in the presence of a solid body, with the vorticity generation step. In fact, the flow adherence condition is violated by the velocity field evaluated in the previous step; therefore a vortex sheet is required on the body contour whose circulation density, $\gamma$, produces the required velocity jump in the tangential velocity:

$$
\gamma\left(x^{\star}\right)=u_{b \tau}\left(x^{\star}\right)-\left(u_{\tau}\left(x^{\star}\right)+u_{\infty \tau}\right), \quad x^{\star} \in \partial \Omega,
$$

where $u_{\tau}$ is perturbation tangent velocity to the body wall evaluated through the integral equation in the first step and $u_{b \tau}$ is the tangential component of the body velocity. Here, $x^{\star}$ represents a generic point on $\partial \Omega$. This source of vorticity will be diffused within the flow field during the following step.

(3) Vorticity diffusion. The viscous effects are accounted for in the Stokes step, where the fluid velocity is neglected and the diffusion of the field vortices as well as of the wall vorticity is evaluated by solving (3-11).

The point vortex approximation can be conveniently inserted into the integral representation for the vorticity field deriving from (3-11). This equation is formally identical to the heat transfer equation and the same fundamental solution can be assumed.

The point vortex approximation (3-13) allows for a very simple and efficient evaluation of the diffusion after one time step $\Delta t_{d}=t-t_{0}$ :

$$
\omega\left(x^{\star}, t\right)=\sum_{j=1}^{N_{\text {vor }}} \frac{\Gamma_{j}\left(t_{0}\right)}{4 \pi v \Delta t_{d}} \exp \left(-\frac{\left|x_{j}-x^{\star}\right|^{2}}{4 v \Delta t_{d}}\right), \quad x^{\star} \in \text { regular meshes. }
$$

Each vortex particle, which is freely moving during the advection step and occupies the position $x_{j}$, gives its diffusive contribution to the nodes $x^{\star}$ of the properly defined grid according to the exact Lamb-Oseen solution. Therefore, after the diffusion step has been completed, the new set of particles located in the nodal positions replaces the former and an automatic and physical redistribution of the vortices occurs (the artificial viscosity due to other interpolation schemes is here absent). A piecewise constant distribution is assumed to translate vorticity into circulation in order to obtain the new vortex particles.

A few words can be spent on the diffusion of the vortex sheet on the body contour. In particular, according to the Chorin-Marsden decomposition [Chorin 1978], the vorticity derivative normal to the wall is assumed to vanish in order to satisfy the 
no-slip condition after the diffusion. Hence the vorticity of the particles close to the body is reflected back into the field in order to satisfy the Neumann condition. The application of the reflection procedure, which is an expression of the image method, is exact only for flat boundaries and gives acceptably approximated results when small curvature values are involved (otherwise the complete expression of the integral representation for the solution must be considered and solved). The vorticity reflection must be enforced only for the particles which are closest to the wall, because beyond a given distance the effect of the body is not felt, and each vortex behaves as in free space. For this reason a boundary fitted grid is assumed around the body in order to evaluate the diffusion of the closest particles while a Cartesian grid is chosen for the diffusion of the remaining ones.

(4) Advection step. The inviscid evolution of the vortical particles is described by the Euler equation (3-12) which states the material behavior of the transported quantity. Therefore, each vortex carries around its circulation without diffusion. In order to evaluate the advection step, the velocity field must be computed to obtain the new position of the vortical particles. The velocity field evaluation turns out to be the most expensive part of the whole numerical solution. In order to satisfy (3-12), each vortex is displaced by the fluid velocity according to

$$
\dot{x}_{j}(t)=u_{j}(t), \quad j=1, \ldots, N_{\mathrm{vor}},
$$

where $x_{j}(t)$ and $u_{j}(t)$ are the unknown position and velocity of each particle at time $t$, respectively. The no-flow boundary condition on the solid walls is associated with (3-18).

The velocity field $u_{j}(t)$ of the new set of vortices obtained by the diffusion step is evaluated using (3-14). In the framework of the adopted VM, the latter can be evaluated as

$$
u_{i}=u_{\infty}+\sum_{\substack{1 \leq j \leq N_{\text {vor }} \\ j \neq i}} \Gamma_{j} K_{\epsilon}\left(x_{i}, x_{j}\right), \quad x_{i} \in \Omega \cup \partial \Omega,
$$

where the $K_{\epsilon}$ is a regularized version of the kernel $K$ (see, for example, [Graziani et al. 1995]). We underline that in (3-19) the summation includes also the vortices on boundary $\partial \Omega$ needed to enforce the boundary conditions $\left(u-u_{b}\right) \cdot n=0$ and $\left(u-u_{b}\right) \cdot \tau=0$. The sum of the incoming flow $u_{\infty}$ and of the perturbation velocity yields the total velocity which allows for the vortex displacement to be evaluated. The operation count for the computation of the velocity of each particle is evidently given by $N_{\text {vor }}^{2}$ if (3-19) is directly used. In order to achieve a faster solution, the flow domain is recursively subdivided and a multipole expansion for the kernel $K$ is adopted with a computational cost of order $N_{\text {vor }} \log \left(N_{\text {vor }}\right)$, which is comparable with the performance of other efficient numerical techniques (for details see 
[Van Dommelen and Rundensteiner 1989]). This step is the most expensive part of the numerical procedure. The same approach has been used for the solution of free surface flows in [Graziani and Landrini 1999].

The numerical integration of (3-18) is performed by means of a fourth-order Runge-Kutta algorithm.

The adopted computational scheme has been shown to converge to the solution of the Navier-Stokes equations with a splitting error proportional to the time step $\Delta t$, which is therefore the relevant quantity for the overall accuracy. The numerical solution is then organized as a repeated sequence of advection, generation, and diffusion steps, each one of which can be solved in the most appropriate way. To be more specific, due to the possibly different advection and diffusion time scales, a suitable value of the time increment could be chosen for each step in order to obtain the required accuracy (for example, the time step for the diffusion could be an integral multiple of that for the advection).

3.2.2. Evaluation of the hydrodynamic loads. In order to analyze the interaction between the flow field and the body motion, the hydrodynamic force must be evaluated. A possible approach is based on the integration of the wall stress vector which can be written for a two-dimensional incompressible flow as

$$
\boldsymbol{F}=\int_{\partial \Omega}(-p n+\mu \omega \tau) d l,
$$

where $n$ is the outer normal to the body and $\tau$ is the anticlockwise tangent.

The pressure field, which is now required in order to evaluate the hydrodynamic force, must be computed as it is described below. By writing the Navier-Stokes equations on the body boundary and by taking the tangential projection, the derivative of the pressure can be expressed as

$$
\frac{1}{\rho} \frac{\partial p}{\partial s}=-\tau \cdot \dot{u}+v \frac{\partial \omega}{\partial n} .
$$

Due to the no-slip condition the fluid acceleration $\dot{u}$ on the wall must coincide with that of the body. The pressure on the body is then obtained by integrating (3-21) and the arbitrary integration constant does not affect the force. On occurrence, the hydrostatic contribution could be added either to the pressure or directly to the resultant force.

Alternatively the hydrodynamic load can be evaluated through the vorticity moments as described in [Graziani and Bassanini 2002].

As it is usual with VMs (see, for example, [Kinney and Cielak 1977]), the last term in (3-21), that is, that related to the vorticity flux from the body, is expressed in terms of the circulation density $\gamma$ generated on the wall during the actual step 
according to

$$
v \frac{\partial \omega}{\partial n}(s)=-\frac{\gamma(s)}{\Delta t} .
$$

To resume, the pressure on the wall is obtained by integrating (3-21), then the force is yielded by an integration according to (3-20).

\section{Numerical results: flow past a fixed circular cylinder}

The classical evolution of the flow past a circular cylinder is here considered. Two Reynolds numbers $\operatorname{Re}=140$ and $\operatorname{Re}=1000$ are simulated both with SPH as well as with the VM described in the previous sections.

Conversely to the VM, for the SPH model the definition of a proper algorithm for the inflow and outflow particles is required (see, for example, [Federico et al. 2012]). Using such algorithm the new inflow particles which enter in the fluid domain are added at the end of the particles' array.

For the VM, since the fluid is treated as incompressible, the flow can be started impulsively without any numerical problems. This is not possible with SPH due to the weakly compressible model adopted. In this case the cylinder is smoothly accelerated with a prescribed time law. The problem is further complicated for SPH by the treatment of the acoustic shock waves, generated during the acceleration stage. The pressure fields at the inflow/outflow boundaries are corrected so as to let the acoustic wave cross them as described in [Lastiwka et al. 2009]. At $\operatorname{Re}=140$ it is well known that the wake is not symmetric and alternate sign vorticity is released, producing the characteristic Kármán vortex sheet. The top portion of Figure 1 shows the streakline obtained experimentally using an electrolytic precipitation of a colloidal smoke [van Dyke 1982]. Thanks to the pure Lagrangian nature of SPH it is very easy to extract the streakline from the wake. Indeed it is sufficient to plot only the upper part of the particles' array which represents the particles that remain for a longer time in the fluid domain before leaving it through the outflow boundary (see the bottom portion of Figure 1).

The SPH simulation has been performed with a spatial resolution of $D / \Delta x=$ 140 , with $D$ being the cylinder diameter and $\Delta x$ the particles' size. The total number of particles required is 4.4 million. In Figure 1 only the first 700,000 particles stored in the array have been plotted with black dots. This kind of visualization is not trivial to obtain with the VM described in the previous section since the diffusion process on the regular mesh destroys the Lagrangian particle path. On the other hand, we show later that the VM allows a prediction of the vorticity field with a larger accuracy with respect to the SPH method. It is important to underline that a small perturbation on the vertical motion of the cylinder has been forced in 

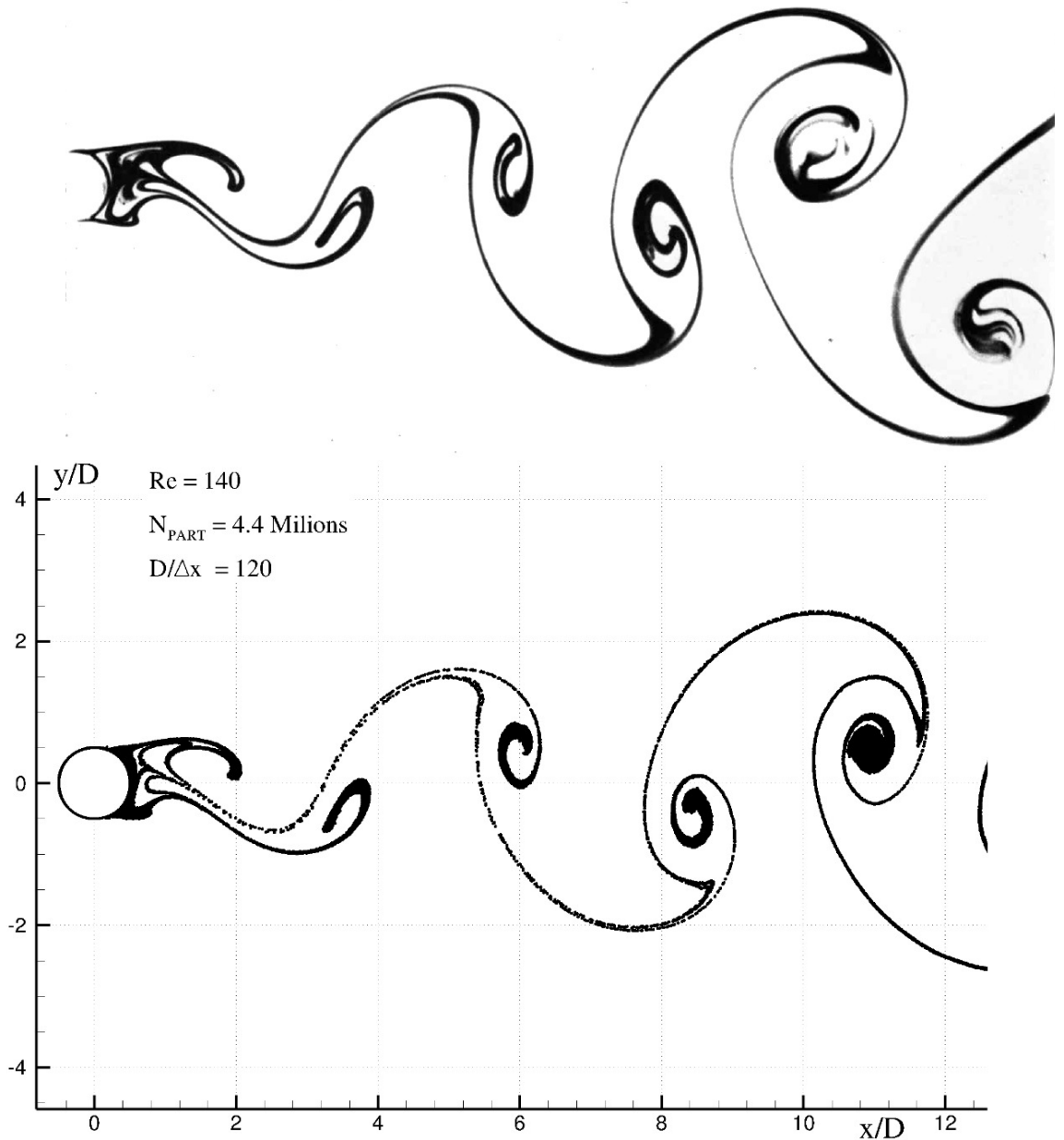

Figure 1. Streakline at $\mathrm{Re}=140$. Top: experimental photograph by Sadatoshi Taneda [van Dyke 1982]. Bottom: SPH simulation.

the vortex model to break the symmetry of the numerical solution and to yield the vortex shedding. This operation is not necessary with the SPH solver for which the symmetric solution is automatically lost after a time which depends on the spatial resolution adopted. The more particles are used, the more time is needed to start the vortex shedding.

Figure 2 shows a comparison between SPH and VM on the drag and lift coefficients. The two different solvers are in fairly good agreement. For the VM the cylinder surface is discretized with 300 panels, therefore the spatial resolution is similar to the SPH one. At the end of the simulation 180,000 vortices are present in the fluid domain. The VM simulation takes one hour on an eight-core CPU (Xeon 

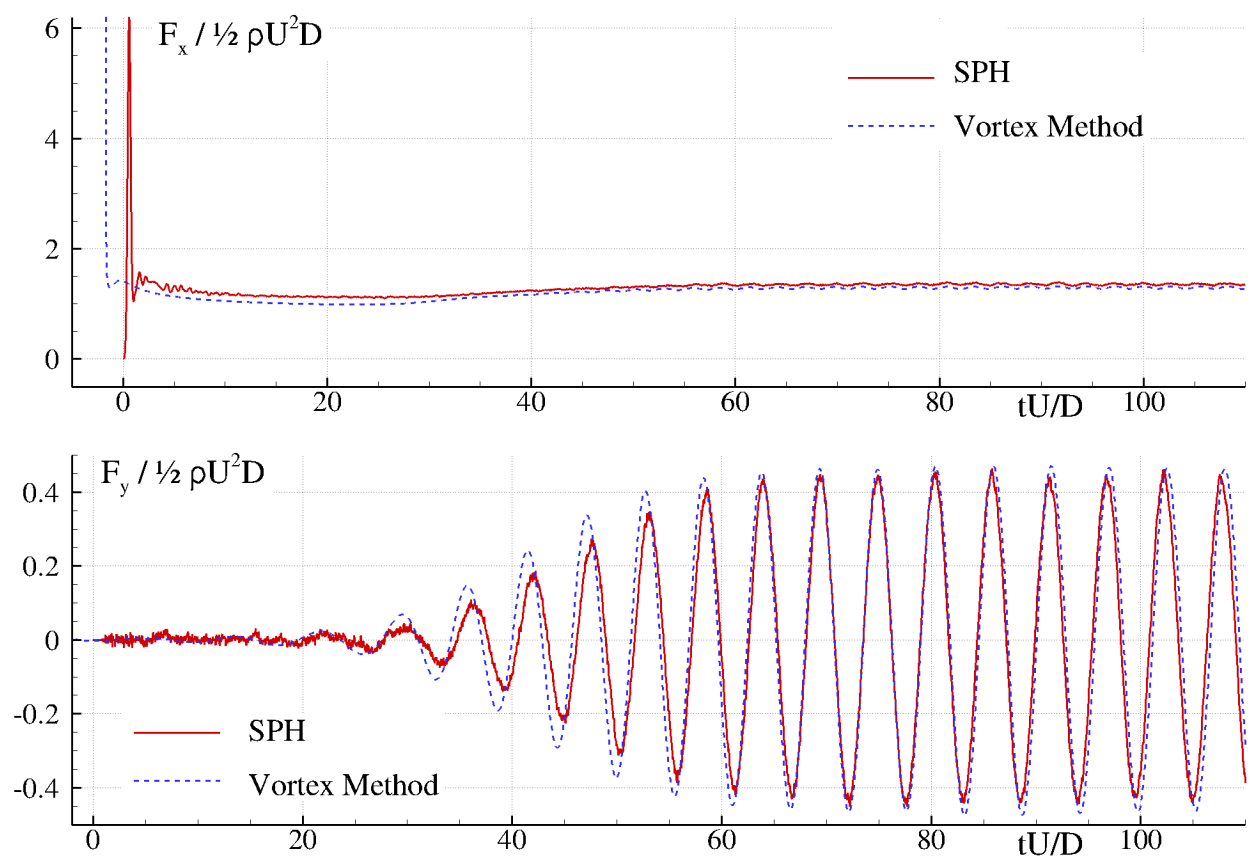

Figure 2. Comparison of the drag and lift forces as predicted by SPH (solid line) and VM (dashed line) for a circular cylinder at $\operatorname{Re}=140$.

E5410 2.33GHz), compared with the four days required for SPH. For this kind of flow SPH has very high CPU costs. However, it must be underlined that the SPH model can easily simulate complex two and three-dimensional violent free-surface flows (see, for example, [Marrone et al. 2011]), which is not the case for the VM.

Finally the case of $\operatorname{Re}=1000$ is considered. Incidentally, we note that twodimensional simulations at this Re value represent a mere numerical test since in reality the flow is fully three-dimensional. Figure 3 shows the vorticity field evaluated by SPH and VM. The SPH vorticity field is quite noisy; besides this the vorticity field of the two solvers are very similar in terms of intensity and shape of the vortical structures.

To solve the flow at this Reynolds number, the spatial resolution has been increased to $D / \Delta x=200$ for SPH and similarly in the VM the cylinder surface has been discretized with 600 panels. The total number of particles adopted for SPH is five million and the simulation takes 26 hours solving a wake 12 diameters long. For the VM the number of vortices at the end of the simulation is 3.3 million, and the simulation takes five days solving a wake 52 diameters long, much longer than the SPH one. The whole wake calculated by the VM is reported in Figure 4 for two different time instants. 

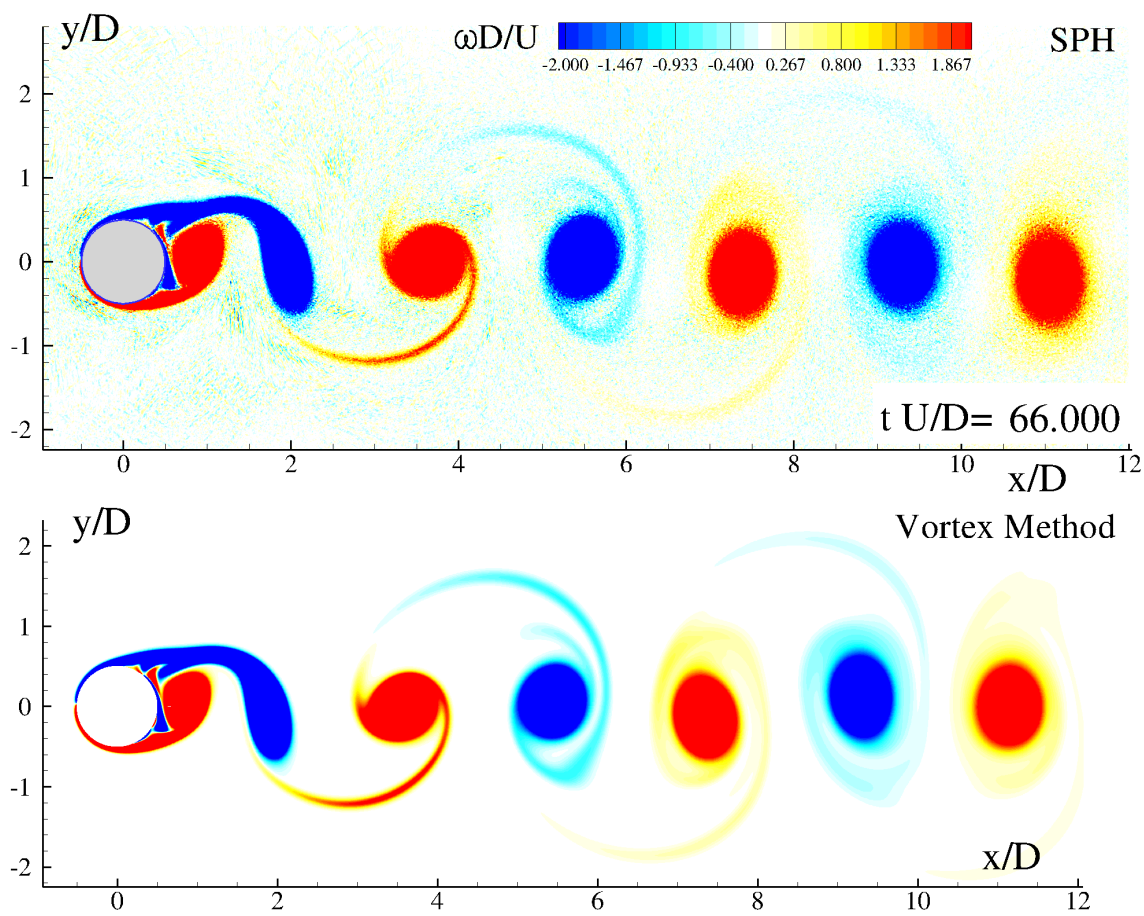

Figure 3. Vorticity field at $\operatorname{Re}=1000$ (two-dimensional simulation) as predicted by SPH (top) and VM (bottom).
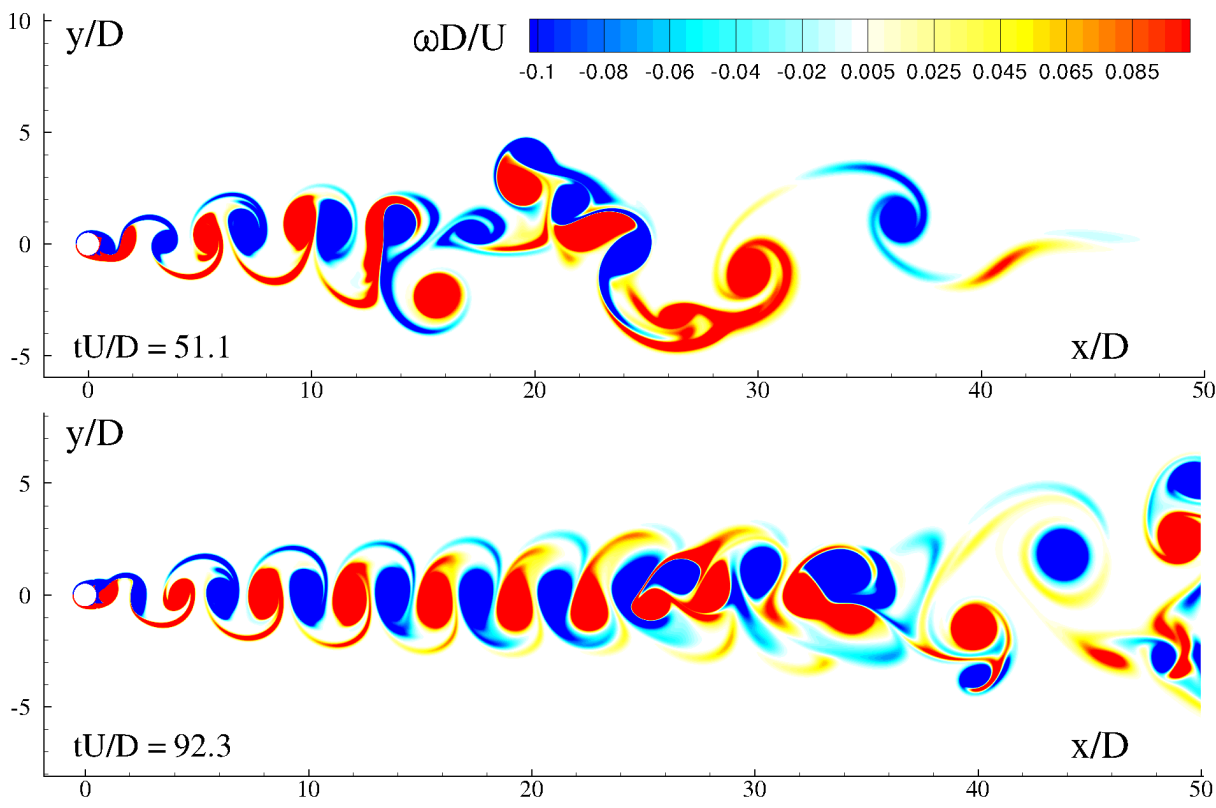

Figure 4. Wake field at $\mathrm{Re}=1000$ (two-dimensional simulation) evaluated by the $\mathrm{VM}$ at times $t U / D=51.1$ and $t U / D=92.3$. 

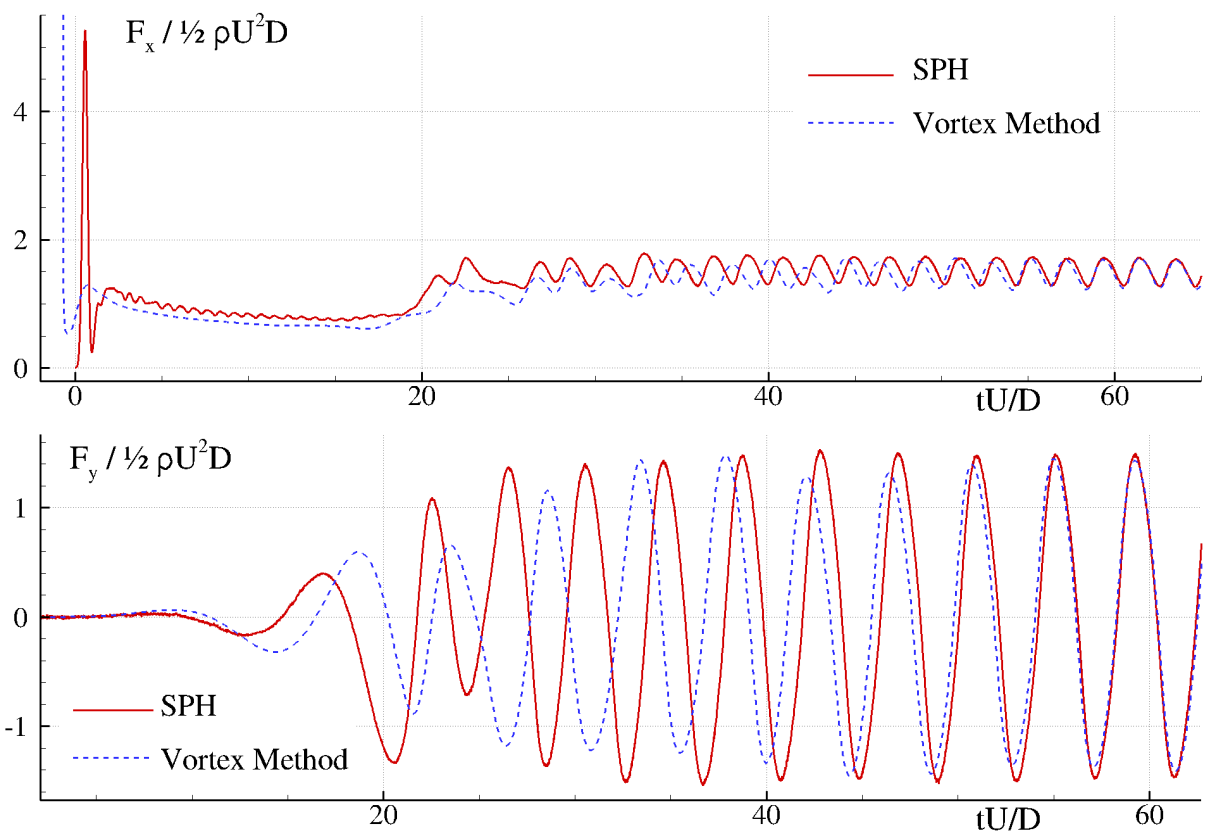

Figure 5. Comparison of the drag and lift forces as predicted by SPH (solid line) and VM (dashed line) for a circular cylinder at $\operatorname{Re}=1000$ (two-dimensional simulation).

The use of different fluid domain lengths adopted for the two solvers reflects on the global loads acting on the cylinder depicted in Figure 5. However, the comparison on the loads remains quite fair when the periodic regime has been reached.

\section{Conclusions}

In this work the use of particle methods for solving fluid dynamic problems is discussed. Models are analyzed theoretically in the context of weakly compressible and incompressible inviscid fluid dynamics. The convergence of the regularized solutions is discussed by using Vlasov dynamics. Numerical formulations for both smoothed-particle hydrodynamics and the vortex model are described for solving viscous weakly compressible and incompressible flows. Application to the flow around a circular cylinder is discussed for both methods considering Reynolds numbers 140 and 1000. The numerical results obtained with the two solvers are compared and the benefits and drawbacks of the different formulations are highlighted. 


\section{Acknowledgements}

The research leading to these results received funding from the Flagship Project RITMARE (Italian Maritime Research), coordinated by the Italian National Research Council and funded by the Italian Ministry of Education, Universities and Research within the National Research Program 2011-2013.

\section{References}

[Antonuccio-Delogu et al. 1996] V. Antonuccio-Delogu, U. Becciani, and F. Magugliani, "Parallelization strategies for tree N-body codes", pp. 24-32 in Applied parallel computing: Computations in physics, chemistry and engineering science, edited by J. Dongarra et al., Lecture Notes in Computer Science 1041, 1996.

[Antuono et al. 2010] M. Antuono, A. Colagrossi, S. Marrone, and D. Molteni, "Free-surface flows solved by means of SPH schemes with numerical diffusive terms", Comput. Phys. Comm. 181:3 (2010), 532-549.

[Barnes and Hut 1986] J. E. Barnes and P. Hut, "A hierarchical $O(N \log N)$ force calculation algorithm”, Nature 324:4 (1986), 446-449.

[Ben Moussa 2006] B. Ben Moussa, "On the convergence of SPH method for scalar conservation laws with boundary conditions", Methods Appl. Anal. 13:1 (2006), 29-61.

[Ben Moussa and Vila 2000] B. Ben Moussa and J. P. Vila, "Convergence of SPH method for scalar nonlinear conservation laws”, SIAM J. Numer. Anal. 37:3 (2000), 863-887.

[Benfatto and Pulvirenti 1984] G. Benfatto and M. Pulvirenti, "Generation of vorticity near the boundary in planar Navier-Stokes flows", Comm. Math. Phys. 96:1 (1984), 59-95.

[Benfatto and Pulvirenti 1986] G. Benfatto and M. Pulvirenti, "Convergence of Chorin-Marsden product formula in the half-plane", Comm. Math. Phys. 106:3 (1986), 427-458.

[Benson et al. 1989] M. G. Benson, P. G. Bellamy-Knights, J. H. Gerrard, and I. Gladwell, "A viscous splitting algorithm applied to low Reynolds number flows round a circular cylinder", Journ. Fluids and Structures 3 (1989), 439-479.

[Boldrighini and Buttà 2011] C. Boldrighini and P. Buttà, "Navier-Stokes equations on a flat cylinder with vorticity production on the boundary", Nonlinearity 24:9 (2011), 2639-2662.

[Braun and Hepp 1977] W. Braun and K. Hepp, "The Vlasov dynamics and its fluctuations in the $1 / N$ limit of interacting classical particles", Comm. Math. Phys. 56:2 (1977), 101-113.

[Chorin 1973] A. J. Chorin, "Numerical study of slightly viscous flow", J. Fluid Mech. 57:4 (1973), 785-796.

[Chorin 1978] A. J. Chorin, "Vortex sheet approximation of boundary layers", J. Comput. Phys. 27 (1978), 428-442.

[Colagrossi and Landrini 2003] A. Colagrossi and M. Landrini, "Numerical simulation of interfacial flows by Smoothed Particle Hydrodynamics", Journal of Computational Physics 191 (2003), 448475 .

[Colagrossi et al. 2009] A. Colagrossi, M. Antuono, and D. Le Touzé, "Theoretical considerations on the free-surface role in the Smoothed Particle Hydrodynamics model”, Physical Review E 79:5 (2009), 056701: 1-13. 
[Colagrossi et al. 2011] A. Colagrossi, M. Antuono, A. Souto-Iglesias, and D. Le Touzé, "Theoretical analysis and numerical verification of the consistency of viscous smoothed-particle-hydrodynamics formulations in simulating free-surface flows", Physical Review E 84 (2011), article id 026705 .

[Colagrossi et al. 2012] A. Colagrossi, B. Bouscasse, M. Antuono, and S. Marrone, "Particle packing algorithm for SPH schemes”, Comput. Phys. Commun. 183:8 (2012), 1641-1653.

[De Leffe et al. 2011] M. De Leffe, D. Le Touzé, and B. Alessandrini, "A modified no-slip condition in weakly-compressible SPH", pp. 291-297 in Proc. 6th International SPHERIC Workshop (Hamburg, 2011), edited by T. Rung and C. Ulrich, Schriftenreihe Schiffbau 658, Hamburg TUHH, 2011.

[Di Lisio 1995] R. Di Lisio, "A particle method for a self-gravitating fluid: A convergence result", Math. Methods Appl. Sci. 18:13 (1995), 1083-1094.

[Di Lisio et al. 1997] R. Di Lisio, E. Grenier, and M. Pulvirenti, "On the regularization of the pressure field in compressible Euler equations”, Ann. Scuola Norm. Sup. Pisa Cl. Sci. (4) 24:2 (1997), 227-238.

[Di Lisio et al. 1998] R. Di Lisio, E. Grenier, and M. Pulvirenti, "The convergence of the SPH method", Comput. Math. Appl. 35:1-2 (1998), 95-102.

[Dobrušin 1979] R. L. Dobrušin, "Vlasov equations", Funktsional. Anal. i Prilozhen. 13:2 (1979), 48-58. In Russian; translated in Funct. Anal. Appl. 13 (1979), 115-123.

[van Dyke 1982] M. van Dyke, An album of fluid motion, Parabolic Press, Stanford, CA, 1982.

[Federico et al. 2012] I. Federico, S. Marrone, A. Colagrossi, F. Aristodemo, and M. Antuono, "Simulating 2D open-channel flows through an SPH model", Eur. J. Mech. B Fluids 34 (2012), $35-46$.

[Graziani and Bassanini 2002] G. Graziani and P. Bassanini, "Unsteady viscous flows about bodies: Vorticity release and forces", Meccanica 37:3 (2002), 283-303.

[Graziani and Landrini 1999] G. Graziani and M. Landrini, "Application of multipoles expansion technique to two-dimensional nonlinear free-surface flows”, J. Ship Res. 43 (1999), 1-12.

[Graziani et al. 1995] G. Graziani, M. Ranucci, and R. Piva, "From a boundary integral formulation to a vortex method for viscous flows", Comput. Mech. 15 (1995), 301-314.

[Kinney and Cielak 1977] R. B. Kinney and Z. M. Cielak, "Analysis of unsteady viscous flow past an airfoil, I: Theoretical development”, AIAA Journal 15 (1977), 1712-1717.

[Koumoutsakos 2005] P. Koumoutsakos, "Multiscale flow simulations using particles", Annu. Rev. Fluid Mech. 37 (2005), 457-487.

[Lastiwka et al. 2009] M. Lastiwka, M. Basa, and N. J. Quinlan, "Permeable and non-reflecting boundary conditions in SPH”, Internat. J. Numer. Methods Fluids 61:7 (2009), 709-724.

[Lucy 1977] L. Lucy, "A numerical approach to testing the fission hypothesis", Astron. J. 82 (1977), 1013.

[Macià et al. 2011] F. Macià, M. Antuono, L. M. Gonzales, and A. Colagrossi, "Theoretical analysis of the no-slip boundary condition enforcement in SPH methods", Prog. Theor. Phys. 125:6 (2011), $1091-1121$.

[Majda 1984] A. Majda, Compressible fluid flow and systems of conservation laws in several space variables, Applied Mathematical Sciences 53, Springer, New York, 1984.

[Marchioro and Pulvirenti 1982] C. Marchioro and M. Pulvirenti, "Hydrodynamics in two dimensions and vortex theory", Comm. Math. Phys. 84:4 (1982), 483-503. 
[Marchioro and Pulvirenti 1984] C. Marchioro and M. Pulvirenti, Vortex methods in two-dimensional fluid dynamics, Lecture Notes in Physics 203, Springer, Berlin, 1984.

[Marchioro and Pulvirenti 1994] C. Marchioro and M. Pulvirenti, Mathematical theory of incompressible nonviscous fluids, Applied Mathematical Sciences 96, Springer, New York, 1994.

[Marrone et al. 2011] S. Marrone, M. Antuono, A. Colagrossi, G. Colicchio, D. Le Touzé, and G. Graziani, " $\delta$-SPH model for simulating violent impact flows", Comput. Methods Appl. Mech. Engrg. 200:13-16 (2011), 1526-1542.

[Marrone et al. 2012] S. Marrone, B. Bouscasse, A. Colagrossi, and M. Antuono, "Study of ship wave breaking patterns using 3D parallel SPH simulations", Comput. Fluids 69 (2012), 54-66.

[Mas-Gallic and Raviart 1987] S. Mas-Gallic and P.-A. Raviart, "A particle method for first-order symmetric systems", Numer. Math. 51:3 (1987), 323-352.

[Molteni and Colagrossi 2009] D. Molteni and A. Colagrossi, "A simple procedure to improve the pressure evaluation in hydrodynamic context using the SPH", Comput. Phys. Comm. 180:6 (2009), 861-872.

[Molteni et al. 2007] D. Molteni, A. Colagrossi, and G. Colicchio, "On the use of an alternative water state equation in SPH”, pp. 23-26 in 2nd SPHERIC International Workshop (Madrid, 2007), edited by A. J. C. Crespo et al., Universidade de Vigo, 2007.

[Monaghan 1992] J. J. Monaghan, "Smoothed particle hydrodynamics", Ann. Rev. of Astron. and Astroph. 30 (1992), 543.

[Monaghan 1994] J. J. Monaghan, "Simulating free surface flows with SPH", J. Comp. Phys 110:2 (1994), 399-406.

[Monaghan and Gingold 1983] J. J. Monaghan and R. A. Gingold, "Shock simulation by the particle method SPH", Journal of Computational Physics 52 (1983), 374-389.

[Neunzert 1984] H. Neunzert, "An introduction to the nonlinear Boltzmann-Vlasov equation", pp. 60-110 in Kinetic theories and the Boltzmann equation (Montecatini, 1981), edited by C. Cercignani, Lecture Notes in Math. 1048, Springer, Berlin, 1984.

[Newton 2001] P. K. Newton, The N-vortex problem: Analytical techniques, Applied Mathematical Sciences 145, Springer, New York, 2001.

[Oelschläger 1991] K. Oelschläger, "On the connection between Hamiltonian many-particle systems and the hydrodynamical equations”, Arch. Rational Mech. Anal. 115:4 (1991), 297-310.

[Swegle et al. 1995] J. W. Swegle, D. L. Hicks, and S. W. Attaway, "Smoothed particle hydrodynamics stability analysis", J. Comput. Phys. 116:1 (1995), 123-134.

[Van Dommelen and Rundensteiner 1989] L. Van Dommelen and E. A. Rundensteiner, "Fast, adaptive summation of point forces in the two dimensional Poisson equation", J. Comput. Phys 83 (1989), 126-147.

Received 5 Nov 2012. Revised 20 Feb 2013. Accepted 4 Apr 2013.

ANDREA COLAGROSSI: andrea.colagrossi@cnr.it

CNR-INSEAN, Via di Vallerano 139, I-00128 Rome, Italy

GIORGIO GRAZIANI: g.graziani@uniroma1.it

Dipartimento di Ingegneria Meccanica e Aerospaziale, Universitá di Roma "La Sapienza",

Via Eudossiana 18, I-00184 Rome, Italy

MARIO PUlVIRENTI: pulviren@mat.uniroma1.it

Dipartimento di Matematica, Universitá di Roma "La Sapienza", Piazzale Aldo Moro 2, I-00185 Rome, Italy 


\title{
NONCLASSICAL CONTINUA, PSEUDOBALANCE, AND THE LAW OF ACTION AND REACTION
}

\author{
GianPietro Del Piero
}

Dedicated to the memory of Luiz-Carlos Martins.

\begin{abstract}
The procedure followed in constructing models for generalized continua is revisited. It is shown that the microforce balance equations required for the description of generalized continua are not in fact expressions of the balance of physical quantities, but consequences of the regularity assumed for the systems of contact actions. In the proposed approach the law of action and reaction, which in classical continuum mechanics is a consequence of Euler's balance laws, recovers the status of a basic principle as held in Newtonian mechanics. Some examples of generalized continua taken from the literature are discussed.
\end{abstract}

\section{Introduction}

In a recent revisitation of the method of virtual power [Del Piero 2009], one of the conclusions was that the two terms - external power and internal powerthat form the equation of virtual power should not be the object of independent assumptions. Once an expression of the external power is assumed, the internal power can be deduced, using some regularity properties of the system of contact actions plus the indifference of the external power.

In the present paper, the roles of regularity and indifference have been separated. Body forces and surface tractions are supposed to be measures, that is, vector-valued set functions additive on disjoint subsets, which we call $\mu$ and $Q$, respectively. The surface tractions form a system of contact actions on the interior surfaces of the body, called a Cauchy flux [Gurtin and Martins 1976]. The regularity assumptions are made on this system, while the indifference of power provides relations between $Q$ and $\mu$. This is the motivation for separating the roles of regularity and indifference.

I thank the anonymous reviewers, whose accurate and keen remarks contributed to improving the overall quality of the paper.

MSC2010: 74-XX.

Keywords: foundations of continuum mechanics, virtual power, generalized continua, microstructure. 
An appropriate set of regularity assumptions on $Q$, which defines what we call a bounded Cauchy flux, provides the general structure required for a self-consistent formulation of continuum mechanics. The first assumption is that the restriction of $Q$ to the collection of the subsurfaces of the boundary of each fixed subbody be an absolutely continuous measure with respect to the area measure. The second is that the restriction of $Q$ to the collection of the boundaries of all subbodies be a measure $F$ absolutely continuous with respect to the volume measure. That is, $Q$ has a surface density $s$, and $F$ has a volume density $f$. Our third, and last, assumption is that $Q$ is skew-symmetric. Thus, $Q$ is defined on oriented surfaces $S$, and the sign of $Q(S)$ changes with the orientation of $S$. In mechanical terms, this corresponds to Newton's law of action and reaction.

The existence of a volume density $f$ for $F$ determines an integral relation between the densities $f$ and $s$, which we call a pseudobalance equation. This equation allows us to prove the dependence of $s$ on the normal to the surface element and the linearity of this dependence. This can be done using two basic tools of continuum mechanics: Noll's theorem on the dependence of $s$ on the normal and Cauchy's tetrahedron theorem. We emphasize that the existence of the Cauchy stress is not deduced, as usual, from the balance law of linear momentum, but from regularity assumptions on the system of contact actions. ${ }^{1}$

It is only at this point that indifference comes into play. It is known that the balance laws of linear and angular momentum can be deduced from the indifference of the external power [Noll 1963]. The first balance law has the same form as the pseudobalance equation, but is of a different nature: as a consequence of indifference, it is a relation between $Q$ and the body force measure $\mu$, and not between surface and volume densities of the same Cauchy flux $Q$. A comparison of the two equations leads to the identification of the volume density $f$ of $F$ with the volume density $b$ of the body force. In this way the equation of virtual power is obtained, and from it a weak formulation of the equilibrium problem can be deduced.

It may look awkward to invent a complicated procedure to end up with no new conclusions. In fact, the advantages of the new procedure become evident when it is extended to more general classes of continua. Essentially, there are two ways to generalize the classical definition of a continuum:

- Relax the regularity assumed for $Q$ and $\mu$, by admitting the presence of singular measures.

- Consider additional external actions, represented by additional measures $Q^{\alpha}, \mu^{\alpha}$. Here we consider generalizations of the second type, which are a standard way to define continua with microstructure. Our basic assumption is that not only $Q$, but

\footnotetext{
${ }^{1}$ This is indeed the main idea in [Gurtin and Martins 1976].
} 
also all $Q^{\alpha}$ are bounded Cauchy fluxes. As a consequence, with each $Q^{\alpha}$ is associated a pseudobalance equation relating the surface density $\sigma^{\alpha}$ to a volume density $\phi^{\alpha}$. But, while $Q$ is related to the body force measure $\mu$ by the balance law of linear momentum, no such relation is assumed to hold between the measures $Q^{\alpha}$ and $\mu^{\alpha}$.

In the literature, the role of the pseudobalance equations is played by microforce balance equations, which are either postulated or deduced from an assumed expression of the internal power. ${ }^{2}$ In both cases it is not clear whether or not these equations are considered as fundamental laws of mechanics, like the balance of linear momentum. If this were the case, a proliferation of postulates would take place, depending on the number and nature of the additional variables. Moreover, in most cases the new postulates would not be based on sound physical motivations.

This is the point where the pseudobalance equations play a basic role. They provide a proof of the existence of counterparts of the stress tensor for the Cauchy fluxes $Q^{\alpha}$, without introducing extra balance laws. This makes possible the transformation of the external power into a volume integral, the internal power. The equality between external and internal power is the equation of virtual power. Note that this equation is not a relation between independent powers, as it is classically considered, but only an identity between equivalent representations of the same power. It turns out that this identity is all that is needed for the formulation of the equilibrium problem.

This paper can be divided into three parts. In the first part, Sections 2-4, some basic concepts of measure theory and geometric measure theory are recalled. The reader not interested in technical details may skip this part. ${ }^{3}$ Continuous bodies are identified with normalized sets of finite perimeter [Šilhavý 1991], and the external actions are limited to the pair $(Q, \mu)$. A proof of Noll's theorem appropriate to this context is given in Appendix A. From it, the pseudobalance equation (4-17) is deduced.

In the second part, Sections 5 and 6, the traditional formulation of continuum mechanics and some alternative approaches are analyzed and compared with the approach based on bounded Cauchy fluxes. The final part, Sections 7 to 11, deals with continua with microstructure. For such continua, general forms of the pseudobalance equations and of the equation of virtual power are given in Section 7. The particular forms taken for specific continua depend on the order parameters which define the microstructure, and on the restrictions due to the indifference of

${ }^{2}$ For the first option see the book [Capriz 1989], in which a law of balance of micromomentum is systematically used. On the second option is based the method of virtual power developed in [Germain 1973b].

${ }^{3}$ For the reader who is interested in such details, a more general introduction can be found in [Vol'pert and Hudjaev 1985]. Basic reference books are [Evans and Gariepy 1992; Ambrosio et al. 2000] for measure theory, and [Federer 1969; Ziemer 1989] for geometric measure theory. 
power, which also vary according to the nature of the continuum. We say that the order parameters define the structural properties of a continuum, not to be confused with the constitutive properties, which characterize specific classes of materials, and which are not considered in this paper.

The structural properties and the indifference requirements determine a subdivision into classes of continua, some of which are discussed in the final sections. Continua in which all measures $Q^{\alpha}$ and $\mu^{\alpha}$ are indifferent to changes of observer are considered in Section 8, and micropolar continua and Cosserat continua are considered in Section 9. Section 10 deals with second-gradient continua. It includes some comments on the edge forces which show up when the contact forces are decomposed into a normal and a tangential part, as required for a correct formulation of the boundary conditions. Finally, Section 11 deals with continua with latent microstructure, characterized by the presence of internal constraints relating the order parameters to the macroscopic deformation.

\section{Basic concepts and definitions}

Let $\Omega$ be a bounded set in the $N$-dimensional Euclidean point space $\mathscr{E} N$, and let $\wp(\Omega)$ be a collection of subsets of $\Omega$, including $\Omega$ and the empty set $\varnothing$. We say that two elements $\Pi_{1}$ and $\Pi_{2}$ of $\wp(\Omega)$ are disjoint if

$$
\Pi \in \wp(\Omega), \quad \Pi \subset \Pi_{1}, \quad \Pi \subset \Pi_{2} \Rightarrow \Pi=\varnothing .
$$

Assume that $\wp(\Omega)$ is equipped with a binary operation $\vee$, which with every pair $\left(\Pi_{1}, \Pi_{2}\right)$ of elements of $\wp(\Omega)$ associates a set $\left(\Pi_{1} \vee \Pi_{2}\right) \in \wp(\Omega)$, called the join of $\Pi_{1}$ and $\Pi_{2}$, such that

$$
\begin{gathered}
\Pi_{1} \subset \Pi_{1} \vee \Pi_{2}, \quad \Pi_{2} \subset \Pi_{1} \vee \Pi_{2}, \\
\Pi_{1} \subset \Pi \text { and } \Pi_{2} \subset \Pi \quad \Rightarrow \quad \Pi_{1} \vee \Pi_{2} \subset \Pi .
\end{gathered}
$$

Assume, further, that with each $\Pi \in \wp(\Omega)$ is associated a set $\Pi^{c} \in \wp(\Omega)$, called the complement of $\Pi$ in $\Omega$, such that

$$
\Pi \text { and } \Pi^{c} \text { are disjoint, } \quad \Pi \vee \Pi^{c}=\Omega .
$$

The join and the complement are unique. ${ }^{4}$ They define a second binary operation

$$
\Pi_{1} \wedge \Pi_{2}=\left(\Pi_{1}^{c} \vee \Pi_{2}^{c}\right)^{c},
$$

called the meet of $\Pi_{1}$ and $\Pi_{2}$. Two sets in $\wp(\Omega)$ are disjoint if and only if their meet is the empty set. ${ }^{5}$

\footnotetext{
${ }^{4} \mathrm{~A}$ proof of this statement and of other statements made in this section can be found in [Del Piero 2003, §5].

${ }^{5}$ The join and the meet are also called, respectively, the least envelope and the greatest common part of $\Pi_{1}$ and $\Pi_{2}$; see [Noll 1973, §8] and [Truesdell 1991, §1.2].
} 
The existence of the join and of the complement provides $\wp(\Omega)$ with the structure of an algebra of sets. This structure includes the following properties:

$$
\begin{array}{ll}
\text { Associative: } & \Pi \vee\left(\Pi_{1} \vee \Pi_{2}\right)=\left(\Pi \vee \Pi_{1}\right) \vee \Pi_{2}, \\
\text { Distributive: } & \Pi \vee\left(\Pi_{1} \wedge \Pi_{2}\right)=\left(\Pi \vee \Pi_{1}\right) \wedge\left(\Pi \vee \Pi_{2}\right),
\end{array}
$$

plus the properties obtained by interchanging $\vee$ and $\wedge$ in the above relations. The set $\bar{\wp}(\Omega)$ of all countable joins and complements of elements of $\wp(\Omega)$ is the $\sigma$-algebra generated by $\wp(\Omega)$.

Let $Y$ be a finite-dimensional inner-product space. ${ }^{6}$ A $Y$-valued measure on $\bar{\wp}(\Omega)$ is a map $\mu: \bar{\wp}(\Omega) \rightarrow Y$, additive on disjoint sets:

$$
\Pi_{1}, \Pi_{2} \in \bar{\wp}(\Omega), \quad \Pi_{1} \wedge \Pi_{2}=\varnothing \quad \Rightarrow \quad \mu\left(\Pi_{1} \vee \Pi_{2}\right)=\mu\left(\Pi_{1}\right)+\mu\left(\Pi_{2}\right) .
$$

Examples of real-valued measures are the $N$-dimensional Lebesgue measure $\mathscr{L}^{N}$ and the $(N-1)$-dimensional Hausdorff measure $\mathscr{H}^{N-1}$. For the former, $\bar{\wp}(\Omega)$ consists of all Lebesgue-measurable subsets of $\mathscr{C}^{N}$; for the latter, it consists of all subsets of $\mathscr{E}^{N}$. For both, $Y$ is the real line, the join and the meet are the union and the intersection of sets, and the complement of $\Pi$ is the complementary set $\Omega \backslash \Pi$. For $N=3$, the two measures are called the volume measure and the area measure, respectively. For convenience we keep these names, and use the notations $V$ and $A$ in place of $\mathscr{L}^{N}$ and $\mathscr{H}^{N-1}$, for every $N$.

A measure $\mu: \bar{\wp}(\Omega) \rightarrow Y$ is absolutely continuous with respect to the volume measure (to the area measure) if $\mu(\Pi)=0$ for all $\Pi \in \bar{\wp}(\Omega)$ for which $V(\Pi)=0$ (for which $A(\Pi)=0$ ). A measure $\mu$ is said to be singular with respect to the same measures if there is a $\Pi_{1} \in \bar{\wp}(\Omega)$ with $V\left(\Pi_{1}\right)=0$ (with $A\left(\Pi_{1}\right)=0$ ) such that $\mu\left(\Pi_{2}\right)=0$ for all $\Pi_{2} \in \bar{\wp}(\Omega)$ disjoint from $\Pi_{1}$. By the Lebesgue decomposition theorem, every measure $\mu$ admits a unique decomposition

$$
\mu=\mu^{a}+\mu^{s},
$$

with $\mu^{a}$ absolutely continuous and $\mu^{s}$ singular with respect to the volume measure (to the area measure).

Let $\Pi$ be a subset of $\mathscr{E}^{N}$, and let $B_{r}(x)$ be the $N$-dimensional ball with radius $r$ centered at $x \in \mathscr{E}^{N}$. The limit

$$
\rho(\Pi, x)=\lim _{r \rightarrow 0} \frac{V\left(B_{r}(x) \wedge \Pi\right)}{V\left(B_{r}(x)\right)},
$$

if it exists, is a real number between 0 and 1. Then $x$ is said to be a point of density for $\Pi$ if $\rho(\Pi, x)=1$, a point of rarefaction if $\rho(\Pi, x)=0$, and a point of the essential boundary otherwise. The set of all density points, the set of all

\footnotetext{
${ }^{6}$ For example, the real line $\mathbb{R}$, the set $\mathbb{R}^{N}$ of all vectors of dimension $N$, or the set $\mathbb{R}^{N \times N}$ of all linear transformations on $\mathbb{R}^{N}(N \times N$ matrices, or second-order tensors $)$.
} 
rarefaction points, and the essential boundary are also called the measure-theoretic interior, exterior, and boundary of $\Pi$. They will be denoted by $\Pi^{*}$, ext* $\Pi$, and $\partial^{*} \Pi$, respectively. For the topological interior, exterior, and boundary of $\Pi$, the inclusions

$$
\text { int } \Pi \subset \Pi^{*}, \quad \operatorname{ext} \Pi \subset \operatorname{ext}^{*} \Pi, \quad \partial \Pi \supset \partial^{*} \Pi,
$$

hold. It is also of interest that

$$
\Pi^{*}=\left(\Pi^{*}\right)^{*}, \quad \operatorname{ext}^{*} \Pi=\operatorname{ext}^{*}\left(\Pi^{*}\right), \quad \partial^{*} \Pi=\partial^{*}\left(\Pi^{*}\right) .
$$

Let $H(x, n)$ be the half-space through $x$ with exterior unit normal $n$. Set:

$$
\rho(\Pi, x, n)=\lim _{r \rightarrow 0} \frac{V\left(B_{r}(x) \wedge \Pi \wedge H(x, n)\right)}{V\left(B_{r}(x)\right)} .
$$

We say that a unit vector $n$ is the measure-theoretic exterior normal, in short, the exterior normal, to $\Pi$ at $x$ if

$$
\rho(\Pi, x, n)=\frac{1}{2}, \quad \rho(\Pi, x,-n)=0 .
$$

The exterior normal, if it exists, is unique. At all points $x$ at which the exterior normal exists, one has

$$
\rho(\Pi, x)=\rho(\Pi, x, n)+\rho(\Pi, x,-n)=\frac{1}{2} .
$$

Therefore, all such points belong to the essential boundary of $\Pi$.

The perimeter of a set is the area of the essential boundary. For all sets of finite perimeter, the following properties hold:

(i) The finite unions and intersections and the complementary sets of sets of finite perimeter are sets of finite perimeter,

(ii) On the essential boundary of a set of finite perimeter the exterior normal exists $A$-almost everywhere, ${ }^{7}$

(iii) For a set $\Pi$ of finite perimeter, $\Pi$ and $\Pi^{*}$ differ by a set of zero volume.

(iv) For a set $\Pi$ of finite perimeter, the divergence theorem

$$
\int_{\partial * \Pi} \varphi(x) \cdot n(x) d A=\int_{\Pi} \operatorname{div} \varphi(x) d V
$$

holds for all Lipschitz continuous functions $\varphi: \mathbb{R}^{N} \rightarrow \mathbb{R}^{N}$ [De Giorgi 1954; Federer 1958; Evans and Gariepy 1992]. ${ }^{8}$

${ }^{7}$ That is, except at most at a set of null area. See [Vol'pert and Hudjaev 1985, §4.5.3]. In general, the topological boundary and measure-theoretic boundary are not area-equivalent, see [Noll and Virga 1988] and [Del Piero 2003, §3].

${ }^{8}$ The regularity assumption on $\varphi$ has been progressively relaxed, see footnote 12 below. 
Note that, for

$$
\varphi(x)=T^{T}(x) v(x),
$$

with $T$ and $v$ sufficiently regular tensor and vector fields, respectively, (2-7) provides the Gauss-Green formula

$$
\int_{\partial^{*} \Pi} T(x) n(x) \cdot v(x) d A=\int_{\Pi}(T(x) \cdot \nabla v(x)+\operatorname{div} T(x) \cdot v(x)) d V .
$$

A set $\Pi$ is normalized if $\Pi=\Pi^{*}$. For normalized sets, it can be proved that the rules (2-1), (2-2), and (2-3) are satisfied if and only if

$$
\Pi_{1} \vee \Pi_{2}=\left(\Pi_{1} \cup \Pi_{2}\right)^{*}, \quad \Pi^{c}=(\Omega \backslash \Pi)^{*}, \quad \Pi_{1} \wedge \Pi_{2}=\Pi_{1} \cap \Pi_{2} .
$$

That is, the join is the normalized union, the complement is the normalized complementary set, and the meet is the normalized intersection, which coincides with the intersection. Moreover, for normalized sets,

$$
\Pi^{c}=\operatorname{ext}^{*} \Pi, \quad \operatorname{ext}^{*}\left(\Pi^{c}\right)=\Pi, \quad \partial^{*}\left(\Pi^{c}\right)=\partial^{*} \Pi .
$$

Let $\Omega$ be a normalized set of finite perimeter, and let $\wp(\Omega)$ be the set of all normalized subsets of $\Omega$ of finite perimeter. Because the join, the meet, and the complement of normalized sets are normalized sets, and the join, the meet, and the complement of sets of finite perimeter are sets of finite perimeter, $\wp(\Omega)$ is an algebra of sets. Its elements will be called the subbodies of $\Omega$. Then, $\bar{\wp}(\Omega)$ is the $\sigma$-algebra generated by the subbodies of $\Omega$.

\section{Surfaces, oriented surfaces, surface measures}

The surface of a subbody $\Pi$ is the essential boundary $\partial^{*} \Pi$. The subsurfaces of $\partial^{*} \Pi$ are the intersections of a surface with other subbodies

$$
S_{1}=\partial^{*} \Pi \cap \Pi_{1}, \quad \Pi_{1} \in \wp(\Omega) .
$$

The relations

$$
\partial^{*} \Pi_{1} \cap \Pi_{2}=\partial^{*}\left(\Pi_{1} \cap \Pi_{2}\right) \cap \Pi_{2}=\partial^{*}\left(\Pi_{1} \vee \Pi_{2}^{c}\right) \cap \Pi_{2}
$$

hold for every pair of subbodies, and the relation

$$
\partial^{*} \Pi_{1} \cap\left(\Pi_{1} \vee \Pi_{2}\right)=\partial^{*} \Pi_{2} \cap\left(\Pi_{1} \vee \Pi_{2}\right)
$$

holds for every pair of disjoint subbodies. The proofs are left to the reader. The subsurface (3-2) is the separating surface of $\Pi_{1}$ and $\Pi_{2}$.

Let $S$ be a subsurface of $\partial^{*} \Pi$. At a point $x$ of $S$, consider the limit

$$
\rho(S, x)=\lim _{r \rightarrow 0} \frac{A\left(S \cap B_{r}(x)\right)}{A_{r}},
$$




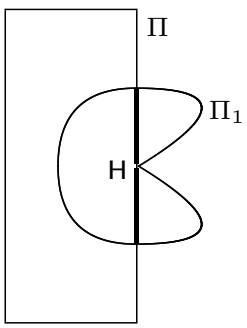

(a)

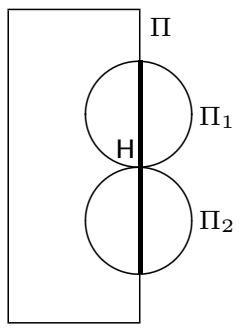

(b)

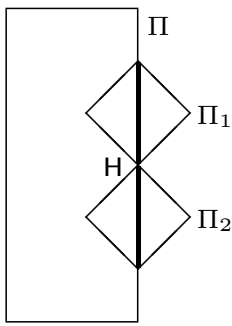

(c)

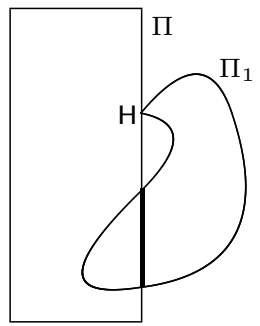

(d)

Figure 1. Two-dimensional examples. In (a), $\partial^{*} \Pi \cap \Pi_{1}$ is a nonnormalized subsurface. In (b) and (c), $\partial^{*} \Pi \cap \Pi_{1}$ and $\partial^{*} \Pi \cap \Pi_{2}$ are two normalized subsurfaces, for which $\partial^{*} \Pi \cap\left(\Pi_{1} \vee \Pi_{2}\right)$ is normalized in (b), but not in (c). In (d), $\partial^{*} \Pi \cap \Pi_{1}$ is a normalized subsurface for which $\partial^{*} \Pi \cap \Pi_{1}^{c}$ is a nonnormalized surface.

where $A_{r}$ is the area of the largest circle in $B_{r}(x)$. For $S=\partial^{*} \Pi$, this limit is equal to one for $A$-almost every $x \in \partial^{*} \Pi$ [Ziemer 1983, §2]. Then for $S \subset \partial^{*} \Pi$ the same limit is a number between zero and one. We say that $x$ is a density point for $S$ if $\rho(S, x)=1$. The set of all density points of $S$ will be denoted by $S^{\star}$.

A subsurface $S$ is normalized if $S=S^{\star}$. The surfaces $S$ and $S^{\star}$ differ at most by a set of area zero. If $\Pi$ and $\Pi_{1}$ are normalized sets, the subsurface $S_{1}=\partial^{*} \Pi \cap \Pi_{1}$ need not be normalized. An example is given in Figure 1a, where the point $\mathrm{H}$ does not belong to $\Pi_{1}$ and, therefore, to $S_{1}$, but belongs to $S_{1}^{\star}$.

In a natural way, the subsurfaces of $\partial^{*} \Pi$ inherit the algebraic structure of $\wp(\Omega)$. For the normalized subsurfaces

$$
S_{1}=\left(\partial^{*} \Pi \cap \Pi_{1}\right)^{\star}, \quad S_{2}=\left(\partial^{*} \Pi \cap \Pi_{2}\right)^{\star},
$$

the join, complement, and meet are defined by

$$
\begin{gathered}
S_{1} \curlyvee S_{2}=\left(\partial^{*} \Pi \cap\left(\Pi_{1} \vee \Pi_{2}\right)\right)^{\star}, \quad S_{1}^{\Pi}=\left(\partial^{*} \Pi \cap \Pi_{1}^{c}\right)^{\star}, \\
S_{1} \curlywedge S_{2}=\left(\partial^{*} \Pi \cap \Pi_{1} \cap \Pi_{2}\right)^{\star},
\end{gathered}
$$

respectively. We say that $S_{1}$ and $S_{2}$ are disjoint if $S_{1} \curlywedge S_{2}=\varnothing$.

If $\Pi, \Pi_{1}$, and $\Pi_{2}$ are normalized, the surfaces $\left(\partial^{*} \Pi \cap\left(\Pi_{1} \vee \Pi_{2}\right)\right),\left(\partial^{*} \Pi \cap \Pi_{1}^{c}\right)$, and $\left(\partial^{*} \Pi \cap \Pi_{1} \cap \Pi_{2}\right)$ need not be normalized. For example, in both Figures $1 b$ and $1 \mathrm{c}$ the point $\mathrm{H}$ is a density point for $\left(\partial^{*} \Pi \cap\left(\Pi_{1} \vee \Pi_{2}\right)\right)$. But in the first case it belongs to $\Pi_{1} \vee \Pi_{2}$ and in the second it does not. In Figure $1 \mathrm{~d}, \mathrm{H}$ is a density point for $\left(\partial^{*} \Pi \cap \Pi_{1}^{c}\right)$ but does not belong to $\Pi_{1}^{c}$. Finally, for $\Pi$ and $\Pi_{1}$ as in Figure 1a and $\Pi_{2}=\Omega$, $\left(\partial^{*} \Pi \cap \Pi_{1} \cap \Pi_{2}\right)$ reduces to $\partial^{*} \Pi \cap \Pi_{1}$, which is not a normalized subsurface. This proves that the meet of subsurfaces does not coincide with the intersection. 
Of fundamental importance are the following properties of the partition of a subbody $\Pi$ into disjoint subbodies $\Pi_{1}$ and $\Pi_{2}$, by means of a separating surface $S$.

Proposition 3.1. Let $\Pi, \Pi_{1}$, and $\Pi_{2}$ be subbodies such that

$$
\Pi=\Pi_{1} \vee \Pi_{2}, \quad \Pi_{1} \cap \Pi_{2}=\varnothing,
$$

let $S$ be the normalized separating surface $S=\left(\partial^{*} \Pi_{1} \cap \Pi\right)^{\star}$, and let

$$
S_{1}=\left(\partial^{*} \Pi_{1} \cap \Pi_{2}^{c}\right)^{\star}, \quad S_{2}=\left(\partial^{*} \Pi_{2} \cap \Pi_{1}^{c}\right)^{\star} .
$$

Then $S, S_{1}$, and $S_{2}$ are pairwise disjoint, and

$$
S_{1} \curlyvee S_{2}=\partial^{*}\left(\Pi_{1} \vee \Pi_{2}\right), \quad S \curlyvee S_{1}=\partial^{*} \Pi_{1}, \quad S \curlyvee S_{2}=\partial^{*} \Pi_{2} .
$$

Proof. By (3-1),

$$
S_{1}=\left(\partial^{*} \Pi_{1} \cap \Pi_{2}^{c}\right)^{\star}=\left(\partial^{*} \Pi \cap \Pi_{2}^{c}\right)^{\star}, \quad S_{2}=\left(\partial^{*} \Pi_{2} \cap \Pi_{1}^{c}\right)^{\star}=\left(\partial^{*} \Pi \cap \Pi_{1}^{c}\right)^{\star} .
$$

Then both $S_{1}$ and $S_{2}$ are subsurfaces of $\partial^{*} \Pi$. By (3-4) and (2-3),

$$
\begin{aligned}
& S_{1} \curlywedge S_{2}=\left(\partial^{*} \Pi \cap \Pi_{1}^{c} \cap \Pi_{2}^{c}\right)^{\star}=\left(\partial^{*} \Pi \cap\left(\Pi_{1} \vee \Pi_{2}\right)^{c}\right)^{\star}=\left(\partial^{*} \Pi \cap \Pi^{c}\right)^{\star}=\varnothing, \\
& S_{1} \curlyvee S_{2}=\left(\partial^{*} \Pi \cap\left(\Pi_{1}^{c} \vee \Pi_{2}^{c}\right)\right)^{\star}=\left(\partial^{*} \Pi \cap\left(\Pi_{1} \cap \Pi_{2}\right)^{c}\right)^{\star}=\left(\partial^{*} \Pi\right)^{\star}=\partial^{*} \Pi,
\end{aligned}
$$

with the second to last equality due to the fact that $\Pi_{1} \cap \Pi_{2}$ is the empty set, and therefore its complement is $\Omega$. This proves (3-7) ${ }_{1}$. Moreover, since both $S$ and $S_{1}$ are subsurfaces of $\partial^{*} \Pi_{1}$, by (3-4) and by the distributive property,

$$
\begin{aligned}
S \curlywedge S_{1}=\left(\partial^{*} \Pi_{1}\right. & \left.\cap\left(\Pi_{1} \vee \Pi_{2}\right) \cap \Pi_{2}^{c}\right)^{\star} \\
= & \left(\partial^{*} \Pi_{1} \cap\left(\left(\Pi_{1} \cap \Pi_{2}^{c}\right) \vee\left(\Pi_{2} \cap \Pi_{2}^{c}\right)\right)\right)^{\star}=\left(\partial^{*} \Pi_{1} \cap \Pi_{1} \cap \Pi_{2}^{c}\right)^{\star}=\varnothing,
\end{aligned}
$$

because both $\Pi_{2} \cap \Pi_{2}^{c}$ and $\partial^{*} \Pi_{1} \cap \Pi_{1}$ are empty. Furthermore,

$$
S \curlyvee S_{1}=\left(\partial^{*} \Pi_{1} \cap\left(\Pi_{1} \vee \Pi_{2} \vee \Pi_{2}^{c}\right)\right)^{\star}=\left(\partial^{*} \Pi_{1}\right)^{\star}=\partial^{*} \Pi_{1},
$$

with the second equality due to $\Pi_{2} \vee \Pi_{2}^{c}=\Omega$. To get $S \curlywedge S_{2}=\varnothing$ and (3-7) 3 note that, by (3-2),

$$
S=\left(\partial^{*} \Pi_{1} \cap \Pi\right)^{\star}=\left(\partial^{*} \Pi_{2} \cap \Pi\right)^{\star},
$$

that is, both $S$ and $S_{2}$ are subsurfaces of $\partial^{*} \Pi_{2}$. Then it is sufficient to repeat the preceding proof, with the subscripts 1 and 2 interchanged.

Another remarkable consequence of the relations (3-1) is the following decomposition of the essential boundary of an intersection. ${ }^{9}$

\footnotetext{
${ }^{9}$ This proposition generalizes a relation proved in [Ziemer 1983] for nonnormalized surfaces, for which (3-8) holds only to within sets of null area.
} 
Proposition 3.2. For every pair $\Pi_{1}, \Pi_{2}$ in $\wp(\Omega)$,

$$
\partial^{*}\left(\Pi_{1} \cap \Pi_{2}\right)=\left(\partial^{*} \Pi_{1} \cap \Pi_{2}\right)^{\star} \curlyvee\left(\partial^{*} \Pi_{2} \cap \Pi_{1}\right)^{\star} .
$$

Proof. By (3-1) 1 ,

$$
\partial^{*} \Pi_{1} \cap \Pi_{2}=\partial^{*}\left(\Pi_{1} \cap \Pi_{2}\right) \cap \Pi_{2}, \quad \partial^{*} \Pi_{2} \cap \Pi_{1}=\partial^{*}\left(\Pi_{1} \cap \Pi_{2}\right) \cap \Pi_{1} .
$$

Then, by (3-3) and (3-4) 1 with $\Pi=\Pi_{1} \cap \Pi_{2}$,

$$
\left(\partial^{*} \Pi_{1} \cap \Pi_{2}\right)^{\star} \curlyvee\left(\partial^{*} \Pi_{2} \cap \Pi_{1}\right)^{\star}=\left(\partial^{*}\left(\Pi_{1} \cap \Pi_{2}\right) \cap\left(\Pi_{2} \vee \Pi_{1}\right)\right)^{\star} .
$$

On the other hand, by (3-3) and (3-4) 2 with $\Pi=\Pi_{1} \cap \Pi_{2}$ and with $\Pi_{1} \vee \Pi_{2}$ in place of $\Pi_{1}$,

$$
\left(\left(\partial^{*}\left(\Pi_{1} \cap \Pi_{2}\right) \cap\left(\Pi_{1} \vee \Pi_{2}\right)\right)^{\star}\right)^{\Pi}=\left(\partial^{*}\left(\Pi_{1} \cap \Pi_{2}\right) \cap\left(\Pi_{1} \vee \Pi_{2}\right)^{c}\right)^{\star} .
$$

The right-hand side is the empty set, because

$$
\partial^{*}\left(\Pi_{1} \cap \Pi_{2}\right) \subset \partial^{*} \Pi_{1} \cup \partial^{*} \Pi_{2}, \quad\left(\Pi_{1} \vee \Pi_{2}\right)^{c}=\Pi_{1}^{c} \cap \Pi_{2}^{c},
$$

and both $\partial^{*} \Pi_{1} \cap \Pi_{1}^{c}$ and $\partial^{*} \Pi_{2} \cap \Pi_{2}^{c}$ are empty. If the complement of a subsurface of $\partial^{*}\left(\Pi_{1} \cap \Pi_{2}\right)$ is empty, the subsurface coincides with the whole surface:

$$
\left(\partial^{*}\left(\Pi_{1} \cap \Pi_{2}\right) \cap\left(\Pi_{1} \vee \Pi_{2}\right)\right)^{\star}=\partial^{*}\left(\Pi_{1} \cap \Pi_{2}\right) .
$$

Then combining with (3-9) the desired relation (3-8) follows.

The surface $\partial^{*} \Pi$ has a natural orientation, with the interior on the side of $\Pi$ and the exterior on the side of $\Pi^{c}$. By $(2-10)_{3}, \partial^{*} \Pi$ can also be viewed as the surface of $\Pi^{c}$, and in this case it has the opposite orientation. The same holds for the subsurfaces $S_{1}=\left(\partial^{*} \Pi \cap \Pi_{1}\right)^{\star}$. For a given orientation of $\partial^{*} \Pi$, with the symbol $\vec{S}$ we denote the subsurface $S$ with the same orientation, and with $\bar{S}$ we denote the same subsurface with the opposite orientation.

By $\vec{S}(\Pi)$ we denote the set of all countable joins and complements of normalized subsurfaces of $\partial^{*} \Pi$, oriented as $\partial^{*} \Pi$. This set is the $\sigma$-algebra generated by the subsurfaces of $\partial^{*} \Pi$. A $Y$-valued surface measure on $\partial^{*} \Pi$ is a function $Q: \overrightarrow{\mathbb{S}}(\Pi) \rightarrow Y$, additive on disjoint surfaces:

$$
Q\left(S_{1} \curlyvee S_{2}\right)=Q\left(S_{1}\right)+Q\left(S_{2}\right), \quad S_{1}, S_{2} \in \overrightarrow{\mathbb{S}}(\Pi), \quad S_{1} \curlywedge S_{2}=\varnothing .
$$

\section{Cauchy fluxes and pseudobalance equations}

Let $\bar{S}(\Omega)$ be the set of all oriented surfaces in $\Omega$ :

$$
\bar{S}(\Omega)=\bigcup_{\Pi \in \bar{\wp}(\Omega)}\{\vec{S}: \vec{S} \in \overrightarrow{\mathbb{S}}(\Pi)\}
$$


A $Y$-valued Cauchy flux on $\Omega$ is a function $Q: \bar{S}(\Omega) \rightarrow Y$, whose restriction to each $\vec{S}(\Pi)$ is a surface measure on $\partial^{*} \Pi$. A Cauchy flux is symmetric if

$$
Q(\vec{S})=Q(\bar{S}) \text { for all } \vec{S} \in \bar{S}(\Omega),
$$

and is skew-symmetric if

$$
Q(\vec{S})=-Q(\bar{S}) \text { for all } \vec{S} \in \bar{S}(\Omega) .
$$

An example of a real-valued symmetric Cauchy flux is the area measure. In the rest of the paper, we will be interested in skew-symmetric Cauchy fluxes. For them, the following additivity property for nondisjoint surfaces holds [Noll 1973, §8].

Proposition 4.1. For a Cauchy flux $Q$, the equation

$$
Q\left(\partial^{*}\left(\Pi_{1} \vee \Pi_{2}\right)\right)=Q\left(\partial^{*} \Pi_{1}\right)+Q\left(\partial^{*} \Pi_{2}\right)
$$

holds for all pairs $\Pi_{1}, \Pi_{2}$ of disjoint subbodies, if and only if $Q$ is skew-symmetric. Proof. Let $\vec{S}$ be the subsurface $\left(\partial^{*} \Pi_{1} \cap\left(\Pi_{1} \vee \Pi_{2}\right)\right)^{\star}$ oriented as $\partial^{*} \Pi_{1}$, and let $\vec{S}_{1}$ and $\vec{S}_{2}$ be the subsurfaces (3-6) oriented as $\partial^{*} \Pi_{1}$ and $\partial^{*} \Pi_{2}$, respectively. By Proposition 3.1 the three surfaces are pairwise disjoint, and equations (3-7), now rewritten as

$$
\vec{S}_{1} \curlyvee \vec{S}_{2}=\partial^{*}\left(\Pi_{1} \vee \Pi_{2}\right), \quad \vec{S} \curlyvee \vec{S}_{1}=\partial^{*} \Pi_{1}, \quad \bar{S} \curlyvee \vec{S}_{2}=\partial^{*} \Pi_{2},
$$

hold. By the additivity property (3-10) on disjoint surfaces,

$$
\begin{gathered}
Q\left(\partial^{*}\left(\Pi_{1} \vee \Pi_{2}\right)\right)=Q\left(\vec{S}_{1}\right)+Q\left(\vec{S}_{2}\right), \\
Q\left(\partial^{*} \Pi_{1}\right)=Q(\vec{S})+Q\left(\vec{S}_{1}\right), \quad Q\left(\partial^{*} \Pi_{2}\right)=Q(\grave{S})+Q\left(\vec{S}_{2}\right) .
\end{gathered}
$$

Then (4-1) holds if and only if $Q(\vec{S})=-Q(\bar{S})$.

Let $Q$ be a Cauchy flux, and let $F: \bar{\wp}(\Omega) \rightarrow Y$ be the function

$$
F(\Pi) \doteq-Q\left(\partial^{*} \Pi\right) \text {. }
$$

We are interested in whether $F$ is a measure. The question is answered as follows:

Proposition 4.2. Let $Q: \bar{S}(\Omega) \rightarrow Y$ be a Cauchy flux, and let $F$ be as in (4-2). Then $F$ is a $Y$-valued measure on $\bar{\wp}(\Omega)$ if and only if $Q$ is skew-symmetric.

Proof. It is sufficient to prove that $F$ is additive on disjoint subsets:

$$
F\left(\Pi_{1} \vee \Pi_{2}\right)=F\left(\Pi_{1}\right)+F\left(\Pi_{2}\right), \quad \Pi_{1}, \Pi_{2} \in \bar{\wp}(\Omega), \quad \Pi_{1} \cap \Pi_{2}=\varnothing .
$$

By (4-2), this is the same as (4-1). By Proposition 4.1, (4-1) holds if and only if $Q$ is skew-symmetric. 
Thus, the restriction of a skew-symmetric Cauchy flux to the surfaces $\partial^{*} \Pi$ of all $\Pi \in \bar{\wp}(\Omega)$ can be identified with a measure $F$ on $\bar{\wp}(\Omega)$. Consider the Lebesgue decomposition of $F$ into the sum of an absolutely continuous and a singular part with respect to the volume measure:

$$
F=F^{a}+F^{s} .
$$

By the Radon-Nikodým theorem (see, e.g., [Ambrosio et al. 2000]), $F^{a}$ has a volume density $f \in L^{1}(\Omega, Y)$ :

$$
F^{a}(\Pi)=\int_{\Pi} f(x) d V \quad \text { for all } \Pi \in \bar{\wp}(\Omega),
$$

while $F^{s}$ is concentrated on a subset of $\Pi$ with zero volume. Moreover, the Cauchy flux $Q$ admits the decomposition $Q=Q^{a}+Q^{s}$, with

$$
Q^{a}(\vec{S})=\int_{S} s\left(x, \partial^{*} \Pi\right) d A \text { for all } S \in \overrightarrow{\mathbb{S}}(\Pi),
$$

where $s\left(\cdot, \partial^{*} \Pi\right) \in L^{1}\left(\partial^{*} \Pi, Y\right)$ is the surface density associated with the restriction of $Q^{a}$ to $\vec{S}(\Pi)$, and $Q^{s}$ is concentrated on a subset of $\partial^{*} \Pi$ with null area measure. Equation (4-2) then takes the form

$$
\int_{\Pi} f(x) d V+F^{s}(\Pi)+\int_{\partial^{*} \Pi} s\left(x, \partial^{*} \Pi\right) d A+Q^{s}\left(\partial^{*} \Pi\right)=0 .
$$

The measure $Q$ is absolutely continuous with respect to the area measure if $Q^{s}=0$, and $F$ is absolutely continuous with respect to the volume measure if $F^{s}=0$. A sufficient condition for the $A$-absolute continuity of $Q$ is the property of area boundedness

$$
|Q(\vec{S})| \leq K A(\vec{S}) \quad \text { for all } \vec{S} \in \bar{S}(\Omega)
$$

with $K$ a positive constant and $|\cdot|$ the norm of $Y$. A sufficient condition for the $V$-absolute continuity of $F$ is the volume boundedness

$$
|F(\Pi)| \leq K V(\Pi) \quad \text { for all } \Pi \in \bar{\wp}(\Omega) .
$$

The less restrictive condition that for every $\Pi \in \bar{\wp}(\Omega)$ there is a nonnegative function $h^{\Pi} \in L^{1}\left(\partial^{*} \Pi, \mathbb{R}\right)$ such that

$$
|Q(\mathscr{Y})| \leq \int_{\mathscr{S}} h^{\Pi}(x) d A \quad \text { for all } \mathscr{Y} \in \overrightarrow{\mathbb{S}}(\Pi),
$$

is necessary and sufficient for the $A$-absolute continuity of $Q$, and the condition that there is a nonnegative function $h \in L^{1}(\Omega, \mathbb{R})$ such that

$$
|F(\Pi)| \leq \int_{\Pi} h(x) d V \quad \text { for all } \Pi \in \bar{\wp}(\Omega),
$$


is necessary and sufficient for the $V$-absolute continuity of $F$ [Šilhavý 1985]. A flux with the properties (4-8) and (4-9) is called a weakly balanced Cauchy flux. ${ }^{10}$

Thus, $F$ is a measure if and only if $Q$ is skew-symmetric, and this measure is $V$-absolutely continuous if and only if $Q$ is weakly balanced. A skew-symmetric weakly balanced Cauchy flux is called a bounded Cauchy flux [Šilhavý 2008]. For such fluxes, (4-2) takes the special form

$$
\int_{\Pi} f(x) d V+\int_{\partial^{*} \Pi} s\left(x, \partial^{*} \Pi\right) d A=0 .
$$

Equation (4-5) and its special form (4-10) will be called pseudobalance equations, to distinguish them from the balance equations of continuum mechanics, which have the same form but a different physical meaning.

The pseudobalance equation (4-10) has two important consequences. For almost every $x \in \Omega$,

(i) the surface density

$$
s\left(x, \partial^{*} \Pi\right)=\lim _{r \rightarrow 0} \frac{Q\left(\partial^{*} \Pi \cap B_{r}(x)\right)}{A\left(\partial^{*} \Pi \cap B_{r}(x)\right)}
$$

only depends on the exterior normal $n$ to $\partial^{*} \Pi$ at $x$,

$$
s\left(x, \partial^{*} \Pi\right)=s(x, n), \quad \text { a.e. } x \in \Omega,
$$

and

(ii) the dependence of $s$ on $n$ is linear.

The first consequence was considered a postulate by Cauchy, and was proved later by Noll [1959]. The second is the object of the celebrated tetrahedron theorem of Cauchy. Formally, they can be stated as follows.

Theorem 4.3. Let $Q$ be a bounded Cauchy flux, and let $\Pi$ be a subbody. Let $x$ be a point of $\partial^{*} \Pi$ at which the measure-theoretic exterior normal $n$ exists, and let $s\left(x, \partial^{*} \Pi\right)$ be the limit (4-11). Then for every other subbody $\Pi^{\prime}$ with $x \in \partial^{*} \Pi^{\prime}$ and with the same normal $n$ at $x$,

$$
s\left(x, \partial^{*} \Pi^{\prime}\right)=s\left(x, \partial^{*} \Pi\right) .
$$

Theorem 4.4. Let $Q$ and $s$ be as above, and let the function $h^{P}$ in equation (4-15) below be integrable. Then there is a mapping $T \in L^{1}\left(\Omega, \mathbb{R}^{N \times N}\right)$ such that

$$
s(x, n)=T(x) n
$$

for all $n \in Y$ and for $V$-almost all $x$ in $\Omega$.

${ }^{10}$ See [Gurtin and Martins 1976], modified in [Šilhavý 1985]. 
The proof of Theorem 4.3 given in Appendix A follows the lines of Noll's original proof [Noll 1959], with modifications dictated by the weaker regularity assumed for the surfaces $\partial^{*} \Pi$. For Theorem 4.4, the original proof based on the tetrahedron argument ${ }^{11}$ is sufficient for the purposes of the present paper. It is known [Noll 1973, §8] that Cauchy's proof relies on the somehow artificial assumption of continuity of the vector field $s(\cdot, n)$. Later, the theorem was extended to integrable functions [Gurtin et al. 1968].

A sufficient condition for integrability is the following. For every fixed direction $n$, let $\mathscr{P}_{\xi}^{n}$ be the plane with normal $n$ and with signed distance $\xi$ from a fixed point $x_{0}$. For an absolutely continuous flux $Q$, the function $s(\cdot, n)$ is integrable on each $\mathscr{P}_{\xi}^{n}$. Then, there is a nonnegative number $h^{P}$, depending on $\xi$, such that

$$
\int_{\mathscr{P}_{\xi}^{n} \cap \Omega}|s(x, n)| d A \leq h^{P}(\xi) A\left(P_{\xi}^{n} \cap \Omega\right) .
$$

Assume that the function $\xi \mapsto h^{P}(\xi)$ is integrable over the real line. If $\Omega$ is bounded, this guarantees the integrability of $s(\cdot, n)$ over $\Omega$ [Kolmogorov and Fomin 1968, $\S 35$, Problem 6].

For a bounded Cauchy flux, using (4-14) and the tensorial version of the divergence theorem (2-7)

$$
\int_{\partial^{*} \Pi} T(x) n d A=\int_{\Pi} \operatorname{div} T(x) d V,
$$

which follows from (2-8) written for a constant $v$, the pseudobalance equation (4-10) takes the form of a volume integral

$$
\int_{\Pi}(f(x)+\operatorname{div} T(x)) d V=0 .
$$

By the arbitrariness of $\Pi$, this implies the local relation

$$
f(x)+\operatorname{div} T(x)=0, \quad \text { a.e. } x \in \Omega,
$$

between the divergence of $T$ and the volume density of the measure $F{ }^{12}$

\section{The traditional approach to continuum mechanics}

In this section we recall the traditional formulation of continuum mechanics. Some alternative formulations present in the literature are summarized in the next section.

${ }^{11}$ See [Cauchy 1823]. For proofs not based on the tetrahedron argument see [Šilhavý 1985; 1990; 1991; 2008; Fosdick and Virga 1989; Marzocchi and Musesti 2003].

${ }^{12}$ Because $f$ is integrable by assumption, not only $T$, but also its divergence is integrable. The regularity of $T$ has been successively relaxed to $L^{p}$ with divergence in $L^{p}$ [Ziemer 1983; Šilhavý 1985], to $L^{\infty}$ with divergence measure [Chen and Torres 2005], to $L^{1}$ with divergence measure [Degiovanni et al. 1999], and to measures with divergence measure [Chen and Frid 1999]. 
Let $\Omega$ be the region of $\mathscr{E}^{N}$ occupied by a continuous body. The interaction of a body with the exterior is assumed to consist of two vector-valued measures, a volumic measure $\mu: \bar{\wp}(\Omega) \rightarrow \mathbb{R}^{N}$, called the distance action, and a surface measure $Q: \vec{S}(\Omega) \rightarrow \mathbb{R}^{N}$, called the contact action. ${ }^{13}$ They are subject to two fundamental axioms, the Euler laws of motion and the cut principle of Euler and Cauchy [Truesdell 1991, §III.1].

The Euler laws are the balance laws of linear momentum and of angular momentum. They state that the total action and the total moment exerted on $\Omega$ by the exterior are zero: ${ }^{14}$

$$
\int_{\Omega} d \mu+\int_{\partial^{*} \Omega} d Q=0, \quad \int_{\Omega} x \times d \mu+\int_{\partial^{*} \Omega} x \times d Q=0 .
$$

The cut principle states that the same balance laws hold for every subbody $\Pi$ of $\Omega$. This hypothesis requires, in particular, that the contact action be defined on all surfaces $\partial^{*} \Pi$ and not only on the boundary $\partial^{*} \Omega$, and that (5-1) holds with $\Omega$ replaced by $\Pi$. In other words, the cut principle requires that $Q$ be a Cauchy flux.

The measures $\mu$ and $Q$ are supposed to have a volume density $b$ and an area density $s\left(\cdot, \partial^{*} \Pi\right)$, respectively. Then the balance laws take the form

$$
\begin{aligned}
& \int_{\Omega} b(x) d V+\int_{\partial^{*} \Omega} s\left(x, \partial^{*} \Omega\right) d A=0, \\
& \int_{\Omega} x \times b(x) d V+\int_{\partial^{*} \Omega} x \times s\left(x, \partial^{*} \Omega\right) d A=0 .
\end{aligned}
$$

By the cut principle, the same laws hold for every subbody $\Pi .{ }^{15}$ Then, using the arbitrariness of $\Pi$, from the first balance law the dependence of $s(x, \cdot)$ on the normal, the action-reaction law

$$
s(x, n)=-s(x,-n),
$$

and the existence of a stress tensor $T$ such that

$$
s(x, n)=T(x) n
$$

are deduced using Theorems 4.3 and 4.4 with $f$ replaced by $b$. (For the deduction of (5-3), see [Noll 1959].) As a consequence, the balance equations (5-2) are

${ }^{13}$ Inertia forces are included in $\mu$; see [Noll 1963, §7].

${ }^{14}$ Here and in the following, we denote with the same symbol $x$ both a point in $\mathscr{E}^{N}$ and the position vector $x-o$ with respect to an origin $o$ chosen once and for all.

${ }^{15}$ In fact, writing (5-2) with $\Omega$ replaced by $\Pi$ requires some physical assumptions. Namely, using the same volume density $b$ for $\Omega$ and for $\Pi$ requires the assumption that the distance actions between parts of the body are negligible. Also, treating the contact actions at the interior surfaces of the body in the same way as the contact actions at the boundary means to exclude any special structure of the body's surface, such as, for example, the structure of the material surfaces studied in [Gurtin and Murdoch 1975]. 
reduced to the local forms

$$
\operatorname{div} T(x)+b(x)=0, \quad T(x)=T^{T}(x), \quad \text { a.e. } x \in \Omega .
$$

These are the local equations of motion, or, in the absence of inertia forces, the local equilibrium equations at the internal points of $\Omega$. After introducing a set $\checkmark$ of virtual displacements $v,(5-5)_{1}$ multiplied by $v$ and integrated over $\Pi$, the Gauss-Green formula (2-8), the relation (5-4), and the symmetry condition (5-5) lead to the equation of virtual power

$$
\int_{\Pi} b(x) \cdot v(x) d V+\int_{\partial^{*} \Pi} s(x, n) \cdot v(x) d A=\int_{\Pi} T(x) \cdot \nabla^{S} v(x) d V
$$

This equation states the equality of the external power of the actions $b$ and $s$ with the internal power, given by the product of the internal force $T$ by the generalized deformation $\nabla^{S} v$. This equation has been deduced from the equilibrium equations (5-5). Conversely, if (5-6) is assumed to hold, (5-5) follows after replacing $s(x, n)$ by $T(x) n$. Thus, (5-6) is an alternative definition of equilibrium. It can be regarded as the weak form of the definition of an equilibrated system of actions.

Assume that the external body forces $b$ are known, that surface tractions $s(x)$ are prescribed on a portion $\partial_{s}^{*} \Omega$ of $\partial^{*} \Omega$, and that null displacements are prescribed on the complement $\left(\partial_{s}^{*} \Omega\right)^{c}$ of $\partial_{s}^{*} \Omega$. Denoting by $\mathscr{V}_{o}$ the set of all virtual displacements which vanish on $\left(\partial_{s}^{*} \Omega\right)^{c}$, from (5-6) written for $\Pi=\Omega$ it follows that

$$
\int_{\Omega} b(x) \cdot v(x) d V+\int_{\partial_{s}^{*} \Omega} s(x) \cdot v(x) d A=\int_{\Omega} T(x) \cdot \nabla^{S} v(x) d V \quad \text { for all } v \in \mathscr{V}_{o} \text {. }
$$

By introducing the constitutive equation of an elastic material ${ }^{16}$

$$
T=g(\nabla u),
$$

the weak form of the equilibrium problem for an elastic body is obtained (see [Ciarlet 1988, Theorem 5.2-1]). For nonelastic bodies the formulation is more complicated, since it requires the introduction of additional variables and of the corresponding generalized forces and evolution equations (see for example, [Halphen and Nguyen 1975]). Nonelastic continua will not be considered in this paper.

\section{Alternative approaches}

The traditional approach to continuum mechanics illustrated in the previous section grew over the centuries, starting from the pioneering work of Newton, Euler,

\footnotetext{
${ }^{16}$ Here and in the following, the constitutive equations are relative to the current deformed configuration of the body, taken as the reference configuration. This is the reason why the kinematical variable is the gradient of the virtual displacement.
} 
and Cauchy. Relatively recent is the realization that the balance laws (5-2) are consequences of a more fundamental physical principle, the indifference, that is, the invariance under changes of observer, of the external power [Noll 1963]. ${ }^{17}$ For the external power given by the left side of (5-6)

$$
P_{\mathrm{ext}}(\Pi, v)=\int_{\Pi} b(x) \cdot v(x) d V+\int_{\partial * \Pi} s(x, n) \cdot v(x) d A,
$$

indifference is expressed by the condition

$$
P_{\mathrm{ext}}(\Pi, v)=P_{\mathrm{ext}}(\Pi, v+a+W(\cdot)),
$$

to be satisfied for all $\Pi \in \wp(\Omega)$, for all vectors $a$, and for all skew-symmetric tensors $W .{ }^{18}$ By the linear dependence of $P_{\text {ext }}$ on $v$, this condition holds if and only if

$$
P_{\text {ext }}(\Pi, a)=0, \quad P_{\text {ext }}(\Pi, W(\cdot))=0,
$$

for all $a$ and for all $W$, and from these conditions the balance equations (5-2) easily follow.

Recently, alternative approaches have appeared in the literature. One of them consists in taking the equation of virtual power (5-6) as a postulate. In this case, the weak formulation (5-7) of the equilibrium problem follows directly from (5-6) written for $\Pi=\Omega$ and $v \in \mathscr{V}_{o}$, and the balance equations (5-2) follow from the same equation written for $v=a$ and $v=W(\cdot)$, respectively. This approach has been largely used to construct models of continua with microstructure [Germain 1973a; 1973b]. Some of its advantages and drawbacks will be discussed later.

Also very common is the variational approach, which consists in minimizing an energy functional, whose Euler equation coincides with (5-7). For an elastic continuum, the energy is

$$
E(v)=\int_{\Omega} w(\nabla v(x)) d V-\int_{\Omega} b(x) \cdot v(x) d V-\int_{\partial \Omega} s(x) \cdot v(x) d A,
$$

where $w$ is the strain energy density, and $T=d w(\nabla v) / d \nabla v$ is the constitutive equation. Thus, in the variational approach the constitutive equation enters from the very beginning. Since it is our intention to keep the equilibrium conditions separate from the constitutive assumptions, the variational approach will not be considered here.

${ }^{17}$ In Lagrangian mechanics, the deduction of the balance of linear momentum from the translational indifference of the Lagrangian is a well-known consequence of Noether's theorem on the correspondence between the indifference properties of a functional and conservation laws; see, for example, [Lanczos 1949, p. 403].

${ }^{18}$ Here $a$ is the rigid translation $a(x)=a$, and $W$ is the skew-symmetric tensor $W x=w \times x$ associated with the rotation vector $w$, see Appendix B. 
In the approach based on the properties of bounded Cauchy fluxes introduced in Section 4, the pseudobalance equation (4-10) holds. A comparison of its local form (4-18) with (5-5) 1 leads to the identification

$$
f=b .
$$

That is, the volume density $f$ of $Q$ coincides with the body force. With this identification, the procedure leading to the equilibrium problem becomes identical to the one followed in the traditional approach.

Thus, at a first glance, the difference of the two approaches looks irrelevant. On the contrary, as pointed out in Section 1, when the external actions involve additional measures $\mu^{\alpha}$ and $Q^{\alpha}$, some problems are met in defining additional microscopic balance equations with the traditional approach. As shown in the following section, the assumption that all $Q^{\alpha}$ are bounded Cauchy fluxes provides a quite general solution to this difficulty.

\section{Nonclassical continua}

A continuum with microstructure is a continuum in which the deformations act on two length scales of different orders of magnitude, macroscopic and microscopic. ${ }^{19}$ The macrodeformation is described by the displacement vector $u$, and the microdeformation is described by a finite number of order parameters $d^{\alpha}$, also called internal variables or state variables, defined on finite-dimensional inner product spaces $Y^{\alpha}$. Each order parameter describes a microstructure, and each set of order parameters describes a continuum with microstructure. Here we will use the terms nonclassical continua and classical continua to denote continua with and without microstructure, respectively.

Just as the macrodeformation $u$ is associated with a pair $(\mu, Q)$ of vector-valued measures describing the macroscopic external actions, to each $d^{\alpha}$ corresponds a pair $\left(\mu^{\alpha}, Q^{\alpha}\right)$ of $Y^{\alpha}$-valued measures, describing the microscopic external actions due to the $\alpha$-th microstructure. We confine our attention to the case in which all measures $\mu^{\alpha}$ are absolutely continuous with respect to the volume measure, and all $Q^{\alpha}$ are absolutely continuous with respect to the area measure. ${ }^{20}$ In this case

\footnotetext{
${ }^{19}$ Historically, the first example of a continuum with microstructure is the continuum with couple stresses [Cosserat and Cosserat 1909]. A short history of successive developments and a broad list of applications can be found in the book [Capriz 1989].

${ }^{20}$ In general, this assumption is too restrictive. Stress concentrations corresponding to singular Cauchy fluxes appear even in some classical problems of linear elasticity. Examples of concentrated contact interactions are discussed in [Podio-Guidugli 2004], and examples of stress fields equilibrated with continuous surface tractions at the boundary and exhibiting, at the interior, stress concentrations on singular surfaces or lines are given in [Lucchesi et al. 2006; 2009]. An appropriate environment for the study of stress concentrations is provided by stress fields with divergence measure, see [Chen and Frid 1999; Degiovanni et al. 1999; Chen and Torres 2005; Šilhavý 2008].
} 
each $\mu^{\alpha}$ has a volume density, the body microforce $\beta^{\alpha}$, each $Q^{\alpha}$ has a surface density, the surface microtraction $\sigma^{\alpha}$, and the external power has the form

$$
P_{\mathrm{ext}}\left(\Pi, v, v^{\alpha}\right)=\int_{\Pi}\left(b \cdot v+\beta^{\alpha} \cdot v^{\alpha}\right) d V+\int_{\partial^{*} \Pi}\left(s \cdot v+\sigma^{\alpha} \cdot v^{\alpha}\right) d A,
$$

where $v^{\alpha}$ are virtual variations of the order parameters $d^{\alpha} \cdot{ }^{21}$

We also assume that $Q$ and all $Q^{\alpha}$ are bounded Cauchy fluxes, and we extend to continua with microstructure the approach based on bounded Cauchy fluxes, described in Section 4 for classical continua.

For a bounded Cauchy flux, the pseudobalance equation (4-10) holds for $Q$, and for each $Q^{\alpha}$ the pseudobalance equation

$$
\int_{\Pi} \phi^{\alpha} d V+\int_{\partial * \Pi} \sigma^{\alpha} d A=0
$$

holds as well, with $\phi^{\alpha}$ the volume density associated with the flux $Q^{\alpha}$. By Theorems 4.3 and 4.4 , the relations

$$
s=T n, \quad \sigma^{\alpha}=\Sigma^{\alpha} n,
$$

and the local pseudobalance equations

$$
\operatorname{div} T+f=0, \quad \operatorname{div} \Sigma^{\alpha}+\phi^{\alpha}=0,
$$

follow, where each $\Sigma^{\alpha}$ is a linear map on the corresponding $Y^{\alpha}{ }^{\alpha 2}$

It is convenient to decompose the volume densities $f$ and $\phi^{\alpha}$ into the sums

$$
f=b-z, \quad \phi^{\alpha}=\beta^{\alpha}-\zeta^{\alpha},
$$

where $z$ and $\zeta^{\alpha}$ measure the deviations of the external body forces $b$ and $\beta^{\alpha}$ from the densities $f$ and $\phi^{\alpha}$.

Using (7-4), (7-5), and the Gauss-Green formula (2-8), the external power (7-1) transforms into the internal power

$$
P_{\text {int }}\left(\Pi, v, v^{\alpha}\right)=\int_{\Pi}\left(z \cdot v+T \cdot \nabla v+\zeta^{\alpha} \cdot v^{\alpha}+\Sigma^{\alpha} \cdot \nabla v^{\alpha}\right) d V .
$$

This is a sum of terms, each of which is the scalar product of an internal force by the corresponding generalized deformation. Therefore, the terms $z$ and $\zeta^{\alpha}$ in (7-5) are characterized as internal forces, and $v$ and $v^{\alpha}$ are the corresponding generalized deformations.

${ }^{21}$ Summation over repeated superscripts $\alpha$ is understood. For simplicity of notation, from here onwards the argument $x$ is omitted.

${ }^{22}$ For $Y^{\alpha}$ equal to $\mathbb{R}, \mathbb{R}^{N}$, and $\mathbb{R}^{N \times N}$, the values $\Sigma^{\alpha}(x)$ are vectors, second-order tensors, and third-order tensors, respectively. 
The difference between the internal power (7-6) and the internal power (5-6) of a classical continuum is not only the presence of microstructural terms. Indeed, in (7-6) there is the extra term $(z \cdot v)$, and the generalized deformation $\nabla^{S} v$ is replaced by $\nabla v$. This is due to the indifference requirements. In all examples to be discussed below, the internal power has the translational indifference property ${ }^{23}$

$$
P_{\text {int }}(\Pi, a, 0)=0,
$$

which implies

$$
z=0,
$$

and, therefore, leads to the same identification $f=b$ found for the classical continuum. On the contrary, the condition of rotational indifference varies according to the physical nature of the order parameters. Therefore, the symmetry of $T$ found for a classical continuum is not preserved, in general, in nonclassical continua. These are the reasons for the differences between (7-6) and (5-6) remarked above.

As a consequence of (7-8), $z$ can be dropped from the list of the internal forces and, consequently, $v$ can be dropped from the list of the generalized deformations. With this modification, the equation of virtual power for a continuum with microstructure takes the form

$$
\begin{aligned}
\int_{\Pi}\left(b \cdot v+\beta^{\alpha} \cdot v^{\alpha}\right) d V+\int_{\partial^{*} \Pi}(s \cdot v & \left.+\sigma^{\alpha} \cdot v^{\alpha}\right) d A \\
& =\int_{\Pi}\left(T \cdot \nabla v+\zeta^{\alpha} \cdot v^{\alpha}+\Sigma^{\alpha} \cdot \nabla v^{\alpha}\right) d V .
\end{aligned}
$$

Just as (5-6) for the classical continuum, this equation defines an equilibrated system of actions for a continuum with microstructure.

The constitutive equations are relations between the internal forces and the generalized deformations

$$
\begin{gathered}
T=\hat{T}\left(\nabla v, v^{\alpha}, \nabla v^{\alpha}\right), \quad \zeta^{\alpha}=\hat{\zeta}^{\alpha}\left(\nabla v, \nu^{\alpha}, \nabla v^{\alpha}\right), \\
\Sigma^{\alpha}=\hat{\Sigma}^{\alpha}\left(\nabla v, v^{\alpha}, \nabla v^{\alpha}\right) .
\end{gathered}
$$

Substituting into (7-9) written for $\Pi=\Omega$ and imposing boundary conditions either on the kinematical variables $u$ and $d^{\alpha}$ or on the corresponding contact actions $s$ and $\sigma^{\alpha}$, a generalization of (5-7) is obtained. This is the weak form of the equilibrium problem for an elastic continuum with microstructure.

In models based on generalizations of the traditional approach, the pseudobalance equation $(7-4)_{1}$ is replaced by the balance equation of linear momentum

${ }^{23}$ Just like (3-7) for classical continua, in the present approach the equation of virtual power is in fact an identity, which holds when all systems of contact actions are strongly balanced Cauchy fluxes. By consequence, the external power is indifferent if and only if the internal power is. In the following, we will systematically impose the indifference of the internal power. 
(5-5) $)_{1}$. Equations identical to (7-4) 2 , called microforce balance equations, are either postulated, ${ }^{24}$ or deduced from the equation of virtual power, assumed as a basic postulate. ${ }^{25}$ In both cases, the way these equations are introduced is not completely satisfactory. In the first case, it is difficult to attribute to such equations, which are so strongly dependent on the number and physical nature of the order parameters, the same status of the balance equation of linear momentum, which is a general law of mechanics. ${ }^{26}$. In the second case, it is not clear how much liberty is allowed in the choice of the internal power, once the external power has been defined. The choice cannot be completely arbitrary, because it must be compatible with the balance laws (5-2). Then there must be a tacit preselection of admissible forms of the internal power, in contrast with the character of a postulate attributed to the equation of virtual power.

On the contrary, the approach based on bounded Cauchy fluxes provides the most general form of the internal power compatible with the assumed external power (7-1), that is, with the chosen order parameters. We say that this choice determines the structural properties of a continuum. Together with the indifference requirements, these properties define specific classes of continua. Within each class, the constitutive equations (7-10) define subclasses of materials. In many models present in the literature, the assumed form of the internal power is a special case of (7-6), resulting from a mix of structural properties and constitutive assumptions. $^{27}$

A bounded Cauchy flux is skew-symmetric, and this is the property from which the pseudobalance equations (4-10) and (7-2) follow. From the foundational viewpoint, I find this fact very intriguing. Indeed, the balance equations of linear and angular momentum are expressions of Newton's first two laws of motion, and the skew-symmetry of $Q$ is an expression of the third law, the law of action and reaction. For classical continua, as seen in Section 5, the third law is a consequence of the first two. Accordingly, in classical continuum mechanics the third law is not considered as a general principle. (See, for example, [Truesdell 1991, §12].) For continua with microstructure, the third law gives a precise status to the microforce balance equations, transforming them into pseudobalance equations. In this respect, the third law recovers the role of a general principle.

${ }^{24}$ See, for example, [Capriz 1989, §8]. It is interesting that the assumption that $z$ is zero while $\zeta^{\alpha}$ need not be zero, made on page 22 of the book, coincide with our conclusions deduced from the indifference of power.

${ }^{25}$ This is the method proposed by Germain. Examples of models constructed in this way are given in [Gurtin 2003, Footnote 1] and in [Del Piero 2012, Chapter 2].

${ }^{26} \mathrm{An}$ alternative is to view the microforce balance equations as constitutive assumptions. To my knowledge, the nature of these equations has never been clearly specified.

${ }^{27}$ In this respect, it is instructive to compare the two models for strain-gradient plasticity of [Gurtin 2003; 2004]; see [Del Piero 2012, §2.4]. 


\section{Continua with indifferent microstructure}

Here and in the following sections, we assume that the translational indifference condition (7-7) holds in general. For the rotational condition, consider first the case in which all microstructures are indifferent:

$$
P_{\text {ext }}(\Pi, W(\cdot), 0)=0 .
$$

Because scalars are invariant under changes of observer, this is the case of all microstructures whose order parameters are scalars. Examples are given by the scalar theories of damage, strain-gradient plasticity, and crystal plasticity. (See, for example, [Del Piero 2012, §2.2].)

The result of this condition applied to the internal power (7-6) is the symmetry of $T$. In this way condition (5-5) 2 , which in the traditional approach was a consequence of the balance of angular momentum, is recovered. There are no restrictions on the virtual velocities $\zeta^{\alpha}$ and $\Sigma^{\alpha}$. In the constitutive equations (7-10), the only change is that now the values of the constitutive function $\hat{T}$ are symmetric secondorder tensors.

\section{Micropolar continua}

Micropolar continua are continua with microstructure whose order parameters $d^{\alpha}$ are vector fields, called directors. They may represent, for example, the orientations of a crystalline lattice or the directions of some crystal defects. Since the directors change their orientation with the body's deformation, the rotational indifference requires the invariance of the internal power under simultaneous rigid rotations of the body and of the directors:

$$
P_{\text {int }}\left(\Pi, W(\cdot), W d^{\alpha}(\cdot)\right)=0 .
$$

From (7-6) with $z=0$ it follows that

$$
\begin{aligned}
0 & =\int_{\Pi}\left(T \cdot W+\zeta^{\alpha} \cdot W d^{\alpha}+\Sigma^{\alpha} \cdot W \nabla d^{\alpha}\right) d V \\
& =W \cdot \int_{\Pi}\left(T+\zeta^{\alpha} \otimes d^{\alpha}+\Sigma^{\alpha} \nabla^{T} d^{\alpha}\right) d V,
\end{aligned}
$$

where $\nabla^{T} d^{\alpha}$ is the transpose of $\nabla d^{\alpha}$. This implies the symmetry of the integrand function. Then $T$ is not symmetric in general, and its skew-symmetric part is

$$
T^{W}=-\left(\Sigma^{\alpha} \nabla^{T} d^{\alpha}+\zeta^{\alpha} \otimes d^{\alpha}\right)^{W} .
$$

We say that $T^{W}$ is the reactive part of the internal force $T$, and that the symmetric part $T^{S}$ is the active part. While $T^{W}$ is a known function of the microstructural 
internal forces $\zeta^{\alpha}$ and $\Sigma^{\alpha}, T^{S}$ has to be determined by a constitutive equation. The internal power takes the form

$$
\begin{aligned}
\int_{\Pi}\left(T^{S} \cdot \nabla v+\zeta^{\alpha} \cdot v^{\alpha}-\left(\Sigma^{\alpha} \nabla^{T} d^{\alpha}+\zeta^{\alpha} \otimes d^{\alpha}\right)^{W} \cdot \nabla v+\Sigma^{\alpha} \cdot \nabla v^{\alpha}\right) d V \\
\quad=\int_{\Pi}\left(T^{S} \cdot \nabla^{S} v+\zeta^{\alpha} \cdot\left(v^{\alpha}-\nabla^{W} v d^{\alpha}\right)+\Sigma^{\alpha} \cdot\left(\nabla v^{\alpha}-\nabla^{W} v \nabla d^{\alpha}\right)\right) d V .
\end{aligned}
$$

It shows that the generalized deformations corresponding to the internal forces $T^{S}$, $\zeta^{\alpha}$, and $\Sigma^{\alpha}$ are

$$
\nabla^{S} v, \quad \psi^{\alpha}=v^{\alpha}-\nabla^{W} v d^{\alpha}, \quad \Psi^{\alpha}=\nabla v^{\alpha}-\nabla^{W} v \nabla d^{\alpha},
$$

respectively. Note that $\psi^{\alpha}$ is the relative rotation between the director $d^{\alpha}$ and the corresponding direction in the deformed body. Thus, the equation of virtual power becomes

$$
\begin{aligned}
\int_{\Pi}\left(b \cdot v+\beta^{\alpha} \cdot v^{\alpha}\right) d V+\int_{\partial^{*} \Pi}\left(s \cdot v+\sigma^{\alpha} \cdot v^{\alpha}\right) d A \\
\quad=\int_{\Pi}\left(T^{S} \cdot \nabla^{S} v+\zeta^{\alpha} \cdot \psi^{\alpha}+\Sigma^{\alpha} \cdot \Psi^{\alpha}\right) d V,
\end{aligned}
$$

and the constitutive equations (7-10) take the form

$$
\begin{gathered}
T^{S}=\hat{T}^{S}\left(\nabla^{S} v, \psi^{\alpha}, \Psi^{\alpha}\right), \quad \zeta^{\alpha}=\hat{\zeta}^{\alpha}\left(\nabla^{S} v, \psi^{\alpha}, \Psi^{\alpha}\right), \\
\Sigma^{\alpha}=\hat{\Sigma}^{\alpha}\left(\nabla^{S} v, \psi^{\alpha}, \Psi^{\alpha}\right) .
\end{gathered}
$$

Equation (9-6) defines an equilibrated system of actions for a micropolar continuum. When coupled with the constitutive equations (9-7), it provides the weak formulation for the equilibrium problem for a micropolar continuum.

Special micropolar continua are the Cosserat continua. In a three-dimensional body, they are characterized by three mutually orthogonal directors $d^{\alpha}$, whose virtual velocities are

$$
v^{\alpha}=\omega \times d^{\alpha},
$$

with $\omega$ a vector field. With this assumption the directors preserve length and mutual orthogonality in all infinitesimal deformations, and $\omega$ measures their common rotation. In the expression (7-1) of the external power, we have

$$
\beta^{\alpha} \cdot v^{\alpha}=\beta^{\alpha} \cdot \omega \times d^{\alpha}=d^{\alpha} \times \beta^{\alpha} \cdot \omega,
$$

and, similarly,

$$
\sigma^{\alpha} \cdot v^{\alpha}=d^{\alpha} \times \sigma^{\alpha} \cdot \omega .
$$


By setting (with $\alpha$ summed)

$$
c=d^{\alpha} \times \beta^{\alpha}, \quad m=d^{\alpha} \times \sigma^{\alpha},
$$

the external power takes the form

$$
P_{\mathrm{ext}}(\Pi, v, \omega)=\int_{\Pi}(b \cdot v+c \cdot \omega) d V+\int_{\partial * \Pi}(s \cdot v+m \cdot \omega) d A .
$$

Here $c$ and $m$ are the body couple and the surface couple, respectively. Thus, due to assumption (9-8), the Cosserat continuum is a micropolar continuum with a single vectorial microstructure.

If all $\sigma^{\alpha}$ are the densities of bounded Cauchy fluxes, by the definition of the cross product of a vector and a tensor given in Appendix B, from (7-3) we get

$$
m=d^{\alpha} \times \sigma^{\alpha}=d^{\alpha} \times\left(\Sigma^{\alpha} n\right)=\left(d^{\alpha} \times \Sigma^{\alpha}\right) n=M n,
$$

where $M=d^{\alpha} \times \Sigma^{\alpha}$ is the couple stress. Then using the Gauss-Green formula and setting

$$
\zeta=c+\operatorname{div} M,
$$

the external power transforms into the internal power

$$
P_{\text {int }}(\Pi, v, \omega)=\int_{\Pi}(T \cdot \nabla v+\zeta \cdot \omega+M \cdot \nabla \omega) d V .
$$

The rotational indifference condition (9-1) now requires that

$$
P_{\text {int }}(\Pi, a \times(\cdot), a)=0,
$$

for all constant vectors $a$. The identity $a \times x=(a \times I) x$ and the relation (B-7) in Appendix B imply

$$
T(x) \cdot \nabla(a \times x)=T(x) \cdot(a \times I)=2 t(x) \cdot a,
$$

with $t$ the vector associated with the skew-symmetric part of $T$. Then, by (9-15) and (9-16),

$$
2 t+\zeta=0
$$

This determines the skew-symmetric part of $T$ in the case of Cosserat continua.

In the macroscopic deformation, the infinitesimal rotation is represented by the vector associated with the skew-symmetric part of $\nabla v$. By (B-8), this vector is one half of $\operatorname{curl} v$. Then, again from (B-7),

$$
T \cdot \nabla v+\zeta \cdot \omega=T^{S} \cdot \nabla^{S} v+2 t \cdot \frac{1}{2} \operatorname{curl} v+\zeta \cdot \omega=T^{S} \cdot \nabla^{S} v+\zeta \cdot\left(\omega-\frac{1}{2} \operatorname{curl} v\right)
$$


Then the internal power further reduces to

$$
P_{\text {int }}(\Pi, v, \omega)=\int_{\Pi}\left(T^{S} \cdot \nabla^{S} v+\zeta \cdot\left(\omega-\frac{1}{2} \operatorname{curl} v\right)+M \cdot \nabla \omega\right) d V,
$$

and the constitutive equations (9-7) take the form

$$
\begin{gathered}
T^{S}=\hat{T}^{S}\left(\nabla^{S} v, \omega-\frac{1}{2} \operatorname{curl} v, \nabla \omega\right), \quad \zeta=\hat{\zeta}\left(\nabla^{S} v, \omega-\frac{1}{2} \operatorname{curl} v, \nabla \omega\right), \\
M=\hat{M}\left(\nabla^{S} v, \omega-\frac{1}{2} \operatorname{curl} v, \nabla \omega\right) .
\end{gathered}
$$

Comparison with the constitutive equations (9-7) shows that the relative rotation $\left(\omega-\frac{1}{2} \operatorname{curl} v\right)$ corresponds to the relative rotation $\psi^{\alpha}$ of the general micropolar continuum.

Again, the equation of virtual power obtained by equating the powers (9-12) and (9-20) defines an equilibrated system of actions for a Cosserat continuum, and this same equation coupled with the constitutive equations (9-21) provides the corresponding weak form of the equilibrium problem.

\section{Second-gradient continua}

A second-gradient continuum is a continuum with microstructure whose unique order parameter is the displacement gradient $\nabla u$ [Toupin 1962; Mindlin 1964; Germain 1973a]. See also [Forte and Vianello 1988; Noll and Virga 1990; Dell'Isola and Seppecher 1997; Podio-Guidugli and Vianello 2010]. The corresponding virtual velocity is $\nabla v$, and the external power has the form

$$
P_{\mathrm{ext}}(\Pi, v)=\int_{\Pi}(b \cdot v+B \cdot \nabla v) d V+\int_{\partial^{*} \Pi}(s \cdot v+S \cdot \nabla v) d A .
$$

If the second-order tensor field $S$ is the surface density of a bounded Cauchy flux, the tensorial version ${ }^{28}$ of Theorems 4.3 and 4.4 ensures the existence of a thirdorder tensor field $\mathbb{T}$ such that

$$
\begin{aligned}
S & =\mathbb{} n, & \operatorname{div} \mathbb{T}+\Phi & =0, \\
S_{i j} & =\mathbb{T}_{i j k} n_{k}, & \mathbb{T}_{i j k, k}+\Phi_{i j} & =0,
\end{aligned}
$$

where $\Phi$ is the volume density associated with the Cauchy flux. The internal power, given by the right-hand side of (7-9), now has the form

$$
P_{\text {int }}(\Pi, v)=\int_{\Pi}((T+Z) \cdot \nabla v+\mathbb{T} \cdot \nabla \nabla v) d V,
$$

where $Z=B-\Phi$ is the gap between the external body microforce $B$ and $\Phi$. The rotational indifference is expressed by condition (6-3) 2 , which now implies the

${ }^{28}$ See, for example, [Del Piero 2009, Appendix]. 
symmetry of $(T+Z)$ :

$$
(T+Z)^{W}=0 .
$$

Then the generalized deformation associated with the internal force $(T+Z)$ is $\nabla^{S} v$ instead of $\nabla v$. Moreover, due to the symmetry of $\nabla \nabla v$ with respect to the last two subscripts, only the part of $\mathbb{T}$ symmetric with respect to the last two subscripts, here denoted by $\mathbb{T}^{\times S}$, contributes to the power. Therefore, for a second-gradient continuum the internal forces are the symmetric tensors $(T+Z)$ and $\mathbb{T}^{\times S}$, and $\nabla^{S} v$ and $\nabla \nabla v$ are the corresponding generalized deformations. For an elastic material, the constitutive equations are

$$
T+Z=\hat{F}\left(\nabla^{S} v, \nabla \nabla v\right), \quad \mathbb{T}^{\times S}=\hat{\mathbb{T}}\left(\nabla^{S} v, \nabla \nabla v\right),
$$

with $\hat{F}$ symmetric, and with $\hat{\mathbb{T}}$ symmetric with respect to the last two subscripts. ${ }^{29}$

The name second-order continuum is due to presence of $\nabla \nabla v$ among the generalized deformations. This presence causes some complication in the formulation of the boundary conditions. Indeed, the displacement gradient at the boundary has a normal and a tangential component, and the tangential component is determined by the values of $v$ at the boundary. Then, boundary conditions of place can be prescribed only to $v$ and to the normal component of $\nabla v$.

The boundary conditions are a part of the strong formulation of the equilibrium problem. For a three-dimensional body $\Omega$, at the boundary $\partial^{*} \Omega$ take an orthonormal local reference frame $\left(e^{\alpha}, e^{n}\right)$, where $e^{\alpha}, \alpha \in\{1,2\}$, are tangent vectors, and $e^{n}$ is the exterior normal $n$. After decomposing the product $S \cdot \nabla v$ into a sum of normal and tangential parts, the Gauss-Green formula applied to $\partial^{*} \Omega$ yields

$$
\begin{aligned}
\int_{\partial^{*} \Omega} S \cdot \nabla v d A & =\int_{\partial^{*} \Omega}\left(S_{i n} v_{i, n}+S_{i \alpha} v_{i, \alpha}\right) d A \\
& =\int_{\partial^{*} \Omega}\left(S_{i n} v_{i, n}-S_{i \alpha, \alpha} v_{i}\right) d A=\int_{\partial^{*} \Omega}\left(S n \cdot \nabla_{n} v-\operatorname{div}_{\alpha} S \cdot v\right) d A .
\end{aligned}
$$

In a similar way, a double application of the Gauss-Green formula provides a wellknown transformation of the second-gradient term [Toupin 1962; Mindlin 1964]:

$$
\begin{aligned}
\int_{\Omega} \mathbb{T} \cdot \nabla \nabla v d V & =\int_{\Omega} \mathbb{T}_{i j k}^{\times S} v_{i, j k} d V=-\int_{\Omega} \mathbb{T}_{i j k, k}^{\times S} v_{i, j} d V+\int_{\partial^{*} \Omega} \mathbb{T}_{i j n}^{\times S} v_{i, j} d A \\
& \left.=\int_{\Omega} \mathbb{T}_{i j k, k j}^{\times S} v_{i} d V+\int_{\partial^{*} \Omega}\left(\mathbb{\mathbb { T }}_{i n n}^{\times S} v_{i, n}+\mathbb{T}_{i \alpha k}^{\times S} n_{k} v_{i, \alpha}\right)-\mathbb{T}_{i n k, k}^{\times S} v_{i}\right) d A \\
& =\int_{\Omega} \mathbb{T}_{i j k, k j}^{\times S} v_{i} d V+\int_{\partial^{*} \Omega}\left(\mathbb{T}_{i n n}^{\times S} v_{i, n}-\left(\mathbb{T}_{i \alpha n, \alpha}^{\times S}+\mathbb{T}_{i n k, k}^{\times S}\right) v_{i}\right) d A .
\end{aligned}
$$

${ }^{29}$ In particular, the constitutive equations are independent of $\nabla^{W} v$. See [Grioli 1960, Equation 19], and [Toupin 1962, Equations 5.1-5.3]. 
By the symmetry of $\mathbb{T}^{\times S}$,

$$
\begin{aligned}
& \mathbb{T}_{i \alpha n, \alpha}^{\times S}=\mathbb{T}_{i n \alpha, \alpha}^{\times S}=\left(\operatorname{div}_{\alpha}\left(\mathbb{T}^{\times S} n\right)\right)_{i}, \\
& \mathbb{T}_{i n k, k}^{\times S}=\mathbb{T}_{i n \alpha, \alpha}^{\times S}+\mathbb{T}_{i n n, n}^{\times S}=\left(\operatorname{div}_{\alpha}\left(\mathbb{T}^{\times S} n\right)\right)_{i}+\left(\nabla_{n}\left(\mathbb{T}^{\times S} n n\right)\right)_{i} .
\end{aligned}
$$

Therefore,

$$
\begin{aligned}
\int_{\Omega} \mathbb{T} \cdot \nabla \nabla v d V=\int_{\Omega} \operatorname{div} \operatorname{div} & \mathbb{T}^{\times S} \cdot v d V+\int_{\partial^{*} \Omega} \mathbb{T}^{\times S} n n \cdot \nabla_{n} v d A \\
& -\int_{\partial^{*} \Omega}\left(2 \operatorname{div}_{\alpha}\left(\mathbb{T}^{\times S} n\right)+\nabla_{n}\left(\mathbb{T}^{\times S} n n\right)\right) \cdot v d A .
\end{aligned}
$$

After the transformation

$$
\int_{\Omega} B \cdot \nabla v d V=-\int_{\Omega} \operatorname{div} B \cdot v d V+\int_{\partial^{*} \Omega} B n \cdot v d A
$$

and a similar transformation for $(T+Z)$, the equation of virtual power eventually takes the form

$$
\begin{aligned}
\int_{\Omega}(b-\operatorname{div} B) \cdot v d V+\int_{\partial^{*} \Omega}\left(S n \cdot \nabla_{n} v+\left(B n+s-\operatorname{div}_{\alpha} S\right) \cdot v\right) d A \\
=\int_{\Omega}\left(\operatorname{div} \operatorname{div} \mathbb{T}^{\times S}-\operatorname{div}(T+Z)\right) \cdot v d V+\int_{\partial^{*} \Omega} \mathbb{T}^{\times S} n n \cdot \nabla_{n} v d A \\
\quad+\int_{\partial^{*} \Omega}\left((T+Z) n-2 \operatorname{div}_{\alpha}\left(\mathbb{T}^{\times S} n\right)-\nabla_{n}\left(\mathbb{T}^{\times S} n n\right)\right) \cdot v d A .
\end{aligned}
$$

From the arbitrariness of $v$, the equilibrium equation

$$
\operatorname{div} \operatorname{div} \mathbb{T}^{\times S}-\operatorname{div}(T+Z)=b-\operatorname{div} B,
$$

at the interior points, and the conditions

$$
\begin{aligned}
\left(\left(\mathbb{T}^{\times S} n\right) n-S n\right) \cdot \nabla_{n} v & =0, \\
\left((T+Z) n-2 \operatorname{div}_{\alpha}\left(\mathbb{T}^{\times S} n\right)-\nabla_{n}\left(\left(\mathbb{T}^{\times S} n\right) n\right)-B n-s+\operatorname{div}_{\alpha} S\right) \cdot v & =0,
\end{aligned}
$$

at the boundary, are deduced. The latter provide the desired forms of the boundary conditions of traction

$$
\begin{aligned}
\left(\mathbb{T}^{\times S} n\right) n & =S n, \\
(T+Z) n-2 \operatorname{div}_{\alpha}\left(\mathbb{T}^{\times S} n\right)-\nabla_{n}\left(\left(\mathbb{T}^{\times S} n\right) n\right) & =B n+s-\operatorname{div}_{\alpha} S,
\end{aligned}
$$

on the portion of the boundary on which the values of the contact actions $S$ and $s$ are prescribed. ${ }^{30}$

${ }^{30}$ These are the equations in [Mindlin 1964], in the improved version of [Bleustein 1967], plus the simplification (10-8) due to the symmetry of $\mathbb{T}^{\times S}$. An interesting interpretation of the boundary conditions in terms of orthofibers is given in [Froiio et al. 2010]. 
The operator $\left(\operatorname{div}_{\alpha}\right)$, obtained in (10-6) and (10-7) when applying the GaussGreen formula to $\partial^{*} \Omega$, must be interpreted in the distributional sense. Then, if the boundary $\partial^{*} \Omega$ has an edge line, that is, a line at which the normal is discontinuous, the terms $\operatorname{div}_{\alpha} S$ in (10-6) and $\operatorname{div}_{\alpha}\left(\mathbb{T}^{\times S} n\right)$ in (10-8) include singularities, called edge forces, represented by forces per unit length applied to the edge line. ${ }^{31}$

In (10-6), the power of the hypertractions $S$ is represented as the sum of the powers of two ordinary tractions, $(S n)$ and $\left(\operatorname{div}_{\alpha} S\right)$, and the latter has singularities at points at which the normal is discontinuous. Some authors believe that the presence of singularities requires a reformulation of Theorems 4.3 and 4.4. This does not seem to be the case, as long as the field $S$ itself is not singular, that is, as long as the Cauchy flux $Q^{\alpha}$ is absolutely continuous with respect to the area measure. Indeed, in this case the tensorial versions of Theorems 4.3 and 4.4 apply. The singularities are merely apparent, because they originate from the representation of the power in a discontinuous local basis. ${ }^{32}$

On the contrary, more regular regions and generalized versions of Noll's and Cauchy's theorems become necessary when the external actions involve singular measures. In spite of some valuable progress, ${ }^{33}$ the construction of a comprehensive, self-consistent theory of higher-order continua in the presence of singular measures is still an open problem.

\section{Continua with latent microstructure}

A continuum with latent microstructure is a continuum subject to internal constraints relating the order parameters to the macroscopic deformation [Capriz 1985; 1989]. By effect of the constraint, the internal forces decompose into the sum of an active part and a reactive part. The latter does not appear in the constitutive equations or in the equilibrium equations.

${ }^{31}$ Similarly, in a third-gradient continuum, concentrated forces, called wedge forces, appear at vertices, and higher-order terms appear in higher-gradient continua. See [Noll and Virga 1988; Di Carlo and Tatone 2001; Podio-Guidugli and Vianello 2010; Dell'Isola et al. 2011]. A characterization of the power of a $n$-th gradient continuum based on the concept of diffused subbody was proposed in [Degiovanni et al. 2007].

${ }^{32}$ As stated in [Noll and Virga 1990], "edge interactions should not be confused with external actions concentrated along curves". The problems caused by writing the power equation in terms of tangential and normal components have a long history. They were perhaps met for the first time in the theory of the bending of plates. The determination of the boundary conditions for this problem kept scientists of the caliber of Poisson, Lagrange, Kirchhoff, and Kelvin busy for a good part of the nineteenth century. For a history of the problem see [Timoshenko 1953].

${ }^{33}$ The problem of the regularity of regions was posed in [Noll and Virga 1990]. Sets with curvature measure have been introduced by [Degiovanni et al. 2006]. For extensions of Theorems 4.3 and 4.4 in the presence of singular measures see [Dell'Isola and Seppecher 1997; Marzocchi and Musesti 2003; Degiovanni et al. 2006; Dell'Isola et al. 2011]. 
An example is the second-gradient continuum described in the preceding section. It has a single tensorial order parameter, whose virtual velocity $V^{\alpha}$ is subject to the constraint

$$
V^{\alpha}=\nabla v
$$

By (10-3), the internal forces are $(T+Z)$ and $\mathbb{T}$, and the active parts are $(T+Z)^{S}$ and $\mathbb{T}^{\times S}$.

A second example is given by the Cauchy-Born hypothesis, by which the orientations of the directors $d^{\alpha}$ are forced to follow the macroscopic deformation. That is, their variations $v^{\alpha}$ must satisfy the internal constraint ${ }^{34}$

$$
v^{\alpha}=\nabla v d^{\alpha} .
$$

In this case, the external power (7-1) takes the form (with $\alpha$ summed)

$$
P_{\mathrm{ext}}(\Pi, v)=\int_{\Pi}\left(b \cdot v+\left(\beta^{\alpha} \otimes d^{\alpha}\right) \cdot \nabla v\right) d V+\int_{\partial^{*} \Pi}\left(s \cdot v+\left(\sigma^{\alpha} \otimes d^{\alpha}\right) \cdot \nabla v\right) d A .
$$

This is a special case of (10-1), with

$$
B=\beta^{\alpha} \otimes d^{\alpha}, \quad S=\sigma^{\alpha} \otimes d^{\alpha} .
$$

The internal power is

$$
P_{\mathrm{int}}(\Pi, v)=\int_{\Pi}\left(\left(T+\Sigma^{\alpha} \nabla^{T} d^{\alpha}+\zeta^{\alpha} \otimes d^{\alpha}\right) \cdot \nabla v+\left(\Sigma^{\alpha} \otimes d^{\alpha}\right) \cdot \nabla \nabla v\right) d V,
$$

with $\zeta^{\alpha}$ as in (7-5) 2 . This is a special case of (10-3), with

$$
Z=\Sigma^{\alpha} \nabla^{T} d^{\alpha}+\zeta^{\alpha} \otimes d^{\alpha}, \quad \mathbb{T}=\Sigma^{\alpha} \otimes d^{\alpha} .
$$

Thus, a micropolar continuum obeying the Cauchy-Born hypothesis is a secondgradient continuum with particular forms for $B, S, Z$, and $\mathbb{T}$.

Another special case is the Cosserat continuum with constrained rotation [Toupin 1964]. For this continuum, the virtual velocities $v^{\alpha}$ are subject to the internal constraint

$$
v^{\alpha}=\nabla^{W} v d^{\alpha} .
$$

By (B-4) and (B-8), this constraint can be put in the equivalent form $v^{\alpha}=\frac{1}{2} \operatorname{curl} v \times d^{\alpha}$. Comparing with (9-8), we see that the internal constraint reduces to

$$
\omega=\frac{1}{2} \operatorname{curl} v \text {. }
$$

${ }^{34}$ If the directors $d^{\alpha}$ form a basis for the underlying space and if $d_{\alpha}$ is the dual basis, this is a special case of (11-1), with $V^{\alpha}=v^{\alpha} \otimes d_{\alpha}$. 
That is, the rotations $\omega$ of all directors coincide with the rotation $\frac{1}{2} \operatorname{curl} v$ in the macroscopic deformation. The external power (9-12) reduces to

$$
P_{\mathrm{ext}}(\Pi, v)=\int_{\Pi}\left(b \cdot v+\frac{1}{2} c \cdot \operatorname{curl} v\right) d V+\int_{\partial^{*} \Pi}\left(s \cdot v+\frac{1}{2} m \cdot \operatorname{curl} v\right) d A,
$$

and the internal power $(9-20)$ reduces to

$$
P_{\text {int }}(\Pi, v)=\int_{\Pi}\left(T^{S} \cdot \nabla^{S} v+\frac{1}{2} M \cdot \nabla \operatorname{curl} v\right) d V .
$$

Therefore, the generalized deformations corresponding to the internal forces $T^{S}$ and $M$ are $\nabla^{S} v$ and $\frac{1}{2} \nabla \operatorname{curl} v$, respectively. The constitutive equations (9-21) become

$$
T^{S}=\hat{T}^{S}\left(\nabla^{S} v, \frac{1}{2} \nabla \operatorname{curl} v\right), \quad M=\hat{M}\left(\nabla^{S} v, \frac{1}{2} \nabla \operatorname{curl} v\right) .
$$

This is still a second-order continuum. Indeed, by (B-8),

$$
\frac{1}{2} M \cdot \operatorname{curl} v=\frac{1}{2} M_{i j} e_{i h k} v_{k, h j}=\mathbb{T} \cdot \nabla \nabla v,
$$

with $\mathbb{T}$ the third-order tensor

$$
\mathbb{T}_{k h j}=\frac{1}{2} M_{i j} e_{i h k} .
$$

Note that $\mathbb{T}$ is skew-symmetric with respect to the first two subscripts. Thus $\mathbb{T}$ has nine independent components, as many as the couple stress tensor $M$. For the internal forces $T, \zeta$, and $M$ in (9-15), the active parts are $T^{S}, 0$, and $M$, and the reactive parts are $T^{W}, \zeta$, and 0 , respectively. The latter do not appear in (11-9) and (11-10).

\section{Appendix A: Proof of Theorem 4.3}

The proof is divided into four steps.

Step 1. For simplicity, write $B_{r}$ in place of $B_{r}(x)$. Consider the definition (4-2) for the set $\Pi \cap B_{r}$ :

$$
F\left(\Pi \cap B_{r}\right)+Q\left(\partial^{*}\left(\Pi \cap B_{r}\right)\right)=0 .
$$

By Proposition 3.2, the set $\partial^{*}\left(\Pi \cap B_{r}\right)$ admits the decomposition (3-8):

$$
\partial^{*}\left(\Pi \cap B_{r}\right)=\left(\Pi \cap \partial^{*} B_{r}\right)^{\star} \curlyvee\left(\partial^{*} \Pi \cap B_{r}\right)^{\star} .
$$

Then, by the additivity property (3-10),

$$
Q\left(\partial^{*}\left(\Pi \cap B_{r}\right)\right)=Q\left(\left(\Pi \cap \partial^{*} B_{r}\right)^{\star}\right)+Q\left(\left(\partial^{*} \Pi \cap B_{r}\right)^{\star}\right),
$$

and the pseudobalance equation reduces to

$$
F\left(\Pi \cap B_{r}\right)+Q\left(\left(\Pi \cap \partial^{*} B_{r}\right)^{\star}\right)+Q\left(\left(\partial^{*} \Pi \cap B_{r}\right)^{\star}\right)=0 .
$$


Step 2. Let us prove that

$$
\lim _{r \rightarrow 0} \frac{F\left(\Pi \cap B_{r}\right)}{A\left(\partial^{*} B_{r}\right)}=0 .
$$

By the property (4-9) of the bounded Cauchy fluxes, there is a positive function $h \in L^{1}(\Omega, \mathbb{R})$ such that

$$
\left.\mid F\left(\Pi \cap B_{r}\right)\right)|=| Q\left(\partial^{*}\left(\Pi \cap B_{r}\right)\right) \mid \leq \int_{\Pi \cap B_{r}} h(x) d V .
$$

Therefore,

$$
\lim _{r \rightarrow 0}\left|\frac{F\left(\Pi \cap B_{r}\right)}{A\left(\partial^{*} B_{r}\right)}\right| \leq \lim _{r \rightarrow 0} \frac{\int_{\Pi \cap B_{r}} h(x) d V}{V\left(\Pi \cap B_{r}\right)} \frac{V\left(\Pi \cap B_{r}\right)}{A\left(\partial^{*} B_{r}\right)} .
$$

The first term on the right converges to a finite value $V$-almost everywhere, by the Lebesgue-Besicovitch differentiation theorem (see, e.g., [Evans and Gariepy 1992]), and the second term converges to zero, because the volume of $B_{r}$ goes to zero faster than the area. Then, (A-2) follows.

Step 3. Let us prove that

$$
\lim _{r \rightarrow 0} \frac{Q\left(\left(\partial^{*} B_{r} \cap \Pi\right)^{\star}\right)}{A\left(\partial^{*} B_{r}\right)}=\lim _{r \rightarrow 0} \frac{Q\left(\left(\partial^{*} B_{r} \cap H\right)^{\star}\right)}{A\left(\partial^{*} B_{r}\right)},
$$

where $H=H(x, n)$ is the half-space with $x$ as a boundary point and with exterior normal $n$. From the identity

$$
\Pi=(\Pi \cap H) \vee\left(\Pi \cap H^{c}\right)
$$

it follows that

$$
\begin{aligned}
& \left(\partial^{*} B_{r} \cap \Pi\right)^{\star}=\left(\partial^{*} B_{r} \cap(\Pi \cap H)\right)^{\star} \curlyvee\left(\partial^{*} B_{r} \cap\left(\Pi \cap H^{c}\right)\right)^{\star}, \\
& \left(\partial^{*} B_{r} \cap H\right)^{\star}=\left(\partial^{*} B_{r} \cap(H \cap \Pi)\right)^{\star} \curlyvee\left(\partial^{*} B_{r} \cap\left(H \cap \Pi^{c}\right)\right)^{\star} .
\end{aligned}
$$

Then it is sufficient to prove that

$$
\lim _{r \rightarrow 0} \frac{Q\left(\left(\partial^{*} B_{r} \cap \Pi \cap H^{c}\right)^{\star}\right)}{A\left(\partial^{*} B_{r}\right)}=0, \quad \lim _{r \rightarrow 0} \frac{Q\left(\left(\partial^{*} B_{r} \cap H \cap \Pi^{c}\right)^{\star}\right)}{A\left(\partial^{*} B_{r}\right)}=0 .
$$

Since $H^{c}=H(x,-n)$, from the definition of the measure-theoretic normal we have

$$
\lim _{r \rightarrow 0} \frac{V\left(B_{r} \cap \Pi \cap H^{c}\right)}{V\left(B_{r}\right)}=0 .
$$

On the other hand, by the coarea formula [Ziemer 1983, Equation (8)]

$$
V\left(B_{r} \cap \Pi \cap H^{c}\right)=\int_{0}^{r} A\left(\partial^{*} B_{\eta} \cap \Pi \cap H^{c}\right) d \eta,
$$


that is, the map $r \mapsto V\left(B_{r} \cap \Pi \cap H^{c}\right)$ is differentiable, and

$$
\frac{d}{d r} V\left(B_{r} \cap \Pi \cap H^{c}\right)=A\left(\partial^{*} B_{r} \cap \Pi \cap H^{c}\right)
$$

for almost every $r$, again by the Lebesgue-Besicovitch theorem. (The same holds for the identity next to (A-7) below.) Since $V\left(B_{r}\right)=O\left(r^{N}\right)$, from (A-5) it follows that $V\left(B_{r} \cap \Pi \cap H^{c}\right)=o\left(r^{N}\right)$, and from (A-6) it follows that $A\left(\partial^{*} B_{r} \cap \Pi \cap H^{c}\right)=$ $o\left(r^{N-1}\right)$. Because $A\left(\partial^{*} B_{r}\right)=O\left(r^{N-1}\right)$, we conclude that

$$
\lim _{r \rightarrow 0} \frac{A\left(\partial^{*} B_{r} \cap \Pi \cap H^{c}\right)}{A\left(\partial^{*} B_{r}\right)}=0 .
$$

In the identity

$$
\lim _{r \rightarrow 0} \frac{Q\left(\left(\partial^{*} B_{r} \cap \Pi \cap H^{c}\right)^{\star}\right)}{A\left(\partial^{*} B_{r}\right)}=\lim _{r \rightarrow 0} \frac{Q\left(\left(\partial^{*} B_{r} \cap \Pi \cap H^{c}\right)^{\star}\right)}{A\left(\left(\partial^{*} B_{r} \cap \Pi \cap H^{c}\right)^{\star}\right)} \lim _{r \rightarrow 0} \frac{A\left(\left(\partial^{*} B_{r} \cap \Pi \cap H^{c}\right)^{\star}\right)}{A\left(\partial^{*} B_{r}\right)},
$$

by the absolute continuity of $Q$, the first limit on the right exists and is finite for $A$-almost every $x$ in $\Omega$. The second limit is zero by (A-7), because a subsurface differs from the corresponding normalized surface at most by a set of area zero. Then $(\mathrm{A}-4)_{1}$ follows. Equation $(\mathrm{A}-4)_{2}$ is proved in the same way.

Step 4. From (A-1), (A-2), and (A-3) it follows that

$$
\lim _{r \rightarrow 0} \frac{Q\left(\left(\partial^{*} \Pi \cap B_{r}\right)^{\star}\right)}{A\left(\partial^{*} B_{r}\right)}=\lim _{r \rightarrow 0} \frac{Q\left(\left(\partial^{*} H \cap B_{r}\right)^{\star}\right)}{A\left(\partial^{*} B_{r}\right)} .
$$

If the first limit exists for some $\Pi$, it also exists for $H$ and, by consequence, it exists for any other $\Pi^{\prime}$ with normal $n$ at $x$. On the other hand,

$$
\lim _{r \rightarrow 0} \frac{Q\left(\left(\partial^{*} \Pi \cap B_{r}\right)^{\star}\right)}{A\left(\partial^{*} B_{r}\right)}=\lim _{r \rightarrow 0} \frac{Q\left(\left(\partial^{*} \Pi \cap B_{r}\right)^{\star}\right)}{A\left(\left(\partial^{*} \Pi \cap B_{r}\right)^{\star}\right)} \lim _{r \rightarrow 0} \frac{A\left(\left(\partial^{*} \Pi \cap B_{r}\right)^{\star}\right)}{A\left(\partial^{*} B_{r}\right)} .
$$

At $A$-almost every $x$ on $\partial^{*} \Pi$, the first limit on the right is equal to $s\left(x, \partial^{*} \Pi\right)$, and the second limit is equal to one [Ziemer 1983, Equation (5)]. By (A-8), the same conclusion holds with $\Pi$ replaced by $H$. This proves that

$$
s\left(x, \partial^{*} \Pi\right)=s\left(x, \partial^{*} H\right)
$$

at all points $x \in \partial^{*} \Pi$ at which the limit $s\left(x, \partial^{*} \Pi\right)$ exists, that is, $A$-almost everywhere on $\partial^{*} \Pi$.

If $x$ is one of such points and if $\Pi^{\prime}$ is another surface such that $x \in \partial^{*} \Pi^{\prime}$ and $n$ is the normal at $x$, in the same way as above it can be proved that

$$
s\left(x, \partial^{*} \Pi^{\prime}\right)=s\left(x, \partial^{*} H\right) .
$$

Then it is possible to denote by $s(x, n)$ the common value of all $s\left(x, \partial^{*} \Pi^{\prime}\right)$, and (4-13) follows from (A-9) and (A-10). 


\section{Appendix B: The cross product of a vector by a tensor}

The cross product of a vector $w$ by a second-order tensor $A$ is the second-order tensor $(w \times A)$ such that ${ }^{35}$

$$
(w \times A) v=w \times(A v)
$$

for all vectors $v$. In components,

$$
(w \times A)_{i j}=e_{i h k} w_{h} A_{k j} .
$$

In particular, $w \times I$ is the second-order tensor such that

$$
(w \times I) v=w \times v
$$

for all $v$. By definition, $(w \times I)$ is the skew-symmetric tensor associated with $w$. In components, for $W=w \times I$ the relations

$$
W_{i j}=e_{i k j} w_{k}, \quad w_{i}=\frac{1}{2} e_{j i k} W_{j k},
$$

hold. In particular, let $V$ and $W$ be the skew-symmetric tensors associated with $v$ and $w$, respectively. Then, by (B-1),

$$
2 w \cdot v=e_{j i k} W_{j k} v_{i}=-(v \times W)_{i i}=-I \cdot(v \times W),
$$

and, by (B-1) and (B-3),

$$
v \times W=(v \times I) W=V W .
$$

Then, by the skew-symmetry of $V$ and $W$,

$$
2 w \cdot v=-I \cdot V W=V \cdot W .
$$

Finally, consider the curl of a vector field $v$

$$
(\operatorname{curl} v)_{h}=e_{h s r} v_{r, s}
$$

By (B-2),

$$
(\operatorname{curl} v \times I)_{i j}=e_{i h k} e_{h s r} v_{r, s} \delta_{k j}=v_{i, j}-v_{j, i}=2\left(\nabla^{W} v\right)_{i j},
$$

that is, $2 \nabla^{W} v$ is the skew-symmetric tensor associated with $\operatorname{curl} v$.

${ }^{35}$ This definition is modified from [Antman and Osborn 1979]. 


\section{References}

[Ambrosio et al. 2000] L. Ambrosio, N. Fusco, and D. Pallara, Functions of bounded variation and free discontinuity problems, Oxford University Press, New York, 2000.

[Antman and Osborn 1979] S. S. Antman and J. E. Osborn, "The principle of virtual work and integral laws of motion”, Arch. Ration. Mech. Anal. 69:3 (1979), 231-262.

[Bleustein 1967] J. L. Bleustein, “A note on the boundary conditions of Toupin's strain-gradient theory”, Int. J. Solids Struct. 3 (1967), 1053-1057.

[Capriz 1985] G. Capriz, "Continua with latent microstructure", Arch. Ration. Mech. Anal. 90 (1985), $43-56$.

[Capriz 1989] G. Capriz, Continua with microstructure, Springer Tracts in Natural Philosophy 35, Springer, Berlin, 1989.

[Cauchy 1823] A.-L. Cauchy, "Recherches sur l'équilibre et le mouvement intérieur des corps solides ou fluides, élastiques ou non élastiques", Bull. Soc. Philomatique (1823), 9-13. Reprinted in Oeuvres complètes 2, pp. 300-304, Cambridge University Press, Cambridge, 2009.

[Chen and Frid 1999] G.-Q. Chen and H. Frid, "Divergence-measure fields and hyperbolic conservation laws", Arch. Ration. Mech. Anal. 147:2 (1999), 89-118.

[Chen and Torres 2005] G.-Q. Chen and M. Torres, "Divergence-measure fields, sets of finite perimeter, and conservation laws", Arch. Ration. Mech. Anal. 175:2 (2005), 245-267.

[Ciarlet 1988] P. G. Ciarlet, Mathematical elasticity, I: Three-dimensional elasticity, Studies in Mathematics and its Applications 20, North-Holland, Amsterdam, 1988.

[Cosserat and Cosserat 1909] E. Cosserat and F. Cosserat, Théorie des corps déformables, Hermann, Paris, 1909.

[De Giorgi 1954] E. De Giorgi, "Su una teoria generale della misura $(r-1)$-dimensionale in uno spazio ad $r$ dimensioni”, Ann. Mat. Pura Appl. (4) 36 (1954), 191-213.

[Degiovanni et al. 1999] M. Degiovanni, A. Marzocchi, and A. Musesti, "Cauchy fluxes associated with tensor fields having divergence measure", Arch. Ration. Mech. Anal. 147 (1999), 197-223.

[Degiovanni et al. 2006] M. Degiovanni, A. Marzocchi, and A. Musesti, "Edge-force densities and second-order powers", Ann. Mat. Pura Appl. (4) 185:1 (2006), 81-103.

[Degiovanni et al. 2007] M. Degiovanni, A. Marzocchi, and A. Musesti, "Virtual powers on diffused subbodies and normal traces of tensor-valued measures", pp. 21-53 in Mathematical modelling of bodies with complicated bulk and boundary behavior, edited by M. Šilhavý, Quad. Mat. 20, Dept. Math., Seconda Univ. Napoli, Caserta, 2007.

[Del Piero 2003] G. Del Piero, "A class of fit regions and a universe of shapes for continuum mechanics”, J. Elasticity 70:1-3 (2003), 175-195.

[Del Piero 2009] G. Del Piero, "On the method of virtual power in continuum mechanics", J. Mech. Mater. Struct. 4 (2009), 281-292.

[Del Piero 2012] G. Del Piero, "On the method of virtual power in the mechanics of non-classical continua", lecture notes to the CISM course "Multiscale modelling of complex materials", Udine, 2012. To appear.

[Dell'Isola and Seppecher 1997] F. dell'Isola and P. Seppecher, "Edge contact forces and quasibalanced power", Meccanica 32:1 (1997), 33-52.

[Dell'Isola et al. 2011] F. dell'Isola, P. Seppecher, and A. Madeo, "Beyond Euler-Cauchy continua: the structure of contact actions in $N$-th gradient generalized continua: a generalization of the Cauchy tetrahedron argument", pp. 17-106 in Variational models and methods in solid and fluid 
mechanics (Udine, 2010), edited by F. dell'Isola and S. Gavrilyuk, CISM Courses and Lectures 535, Springer, Vienna, 2011.

[Di Carlo and Tatone 2001] A. Di Carlo and A. Tatone, "(Iper-)tensioni \& equi-potenza", in XV Congresso AIMETA di Meccanica Teorica e Applicata (Taormina, 2001), edited by G. Augusti, Associazione Italiana di Meccanica Teorica e Applicata, Milan, 2001.

[Evans and Gariepy 1992] L. C. Evans and R. F. Gariepy, Measure theory and fine properties of functions, CRC Press, Boca Raton, FL, 1992.

[Federer 1958] H. Federer, "A note on the Gauss-Green theorem", Proc. Amer. Math. Soc. 9 (1958), $447-451$.

[Federer 1969] H. Federer, Geometric measure theory, Grundlehren der mathematischen Wissenschaften 153, Springer, New York, 1969.

[Forte and Vianello 1988] S. Forte and M. Vianello, "On surface stresses and edge forces", Rend. Mat. Appl. (7) 8:3 (1988), 409-426.

[Fosdick and Virga 1989] R. L. Fosdick and E. G. Virga, "A variational proof of the stress theorem of Cauchy", Arch. Ration. Mech. Anal. 105:2 (1989), 95-103.

[Froiio et al. 2010] F. Froiio, A. Zervos, and I. Vardoulakis, "On natural boundary conditions in linear 2nd-grade elasticity", pp. 211-221 in Mechanics of generalized continua, edited by G. A. Maugin and A. V. Metrikine, Adv. Mech. Math. 21, Springer, New York, 2010.

[Germain 1973a] P. Germain, "La méthode des puissances virtuelles en mécanique des milieux continus, I: Théorie du second gradient”, J. Méc. 12 (1973), 235-274.

[Germain 1973b] P. Germain, "The method of virtual power in continuum mechanics, 2: Microstructure”, SIAM J. Appl. Math. 25 (1973), 556-575.

[Grioli 1960] G. Grioli, "Elasticità asimmetrica”, Ann. Mat. Pura Appl. (4) 50 (1960), 389-417.

[Gurtin 2003] M. E. Gurtin, "On a framework for small-deformation viscoplasticity: free energies, microforces, strain gradients", Int. J. Plast. 19 (2003), 47-90.

[Gurtin 2004] M. E. Gurtin, "A gradient theory of small-deformation isotropic plasticity that accounts for the Burgers vector and for dissipation due to plastic spin", J. Mech. Phys. Solids 52:11 (2004), 2545-2568.

[Gurtin and Martins 1976] M. E. Gurtin and L. C. Martins, "Cauchy's theorem in classical physics", Arch. Ration. Mech. Anal. 60:4 (1976), 305-324.

[Gurtin and Murdoch 1975] M. E. Gurtin and A. I. Murdoch, "A continuum theory of elastic material surfaces", Arch. Ration. Mech. Anal. 57 (1975), 291-323.

[Gurtin et al. 1968] M. E. Gurtin, V. J. Mizel, and W. O. Williams, "A note on Cauchy's stress theorem", J. Math. Anal. Appl. 22 (1968), 398-401.

[Halphen and Nguyen 1975] B. Halphen and Q. S. Nguyen, "Sur les matériaux standards généralisés", J. Méc. 14 (1975), 39-63.

[Kolmogorov and Fomin 1968] A. N. Kolmogorov and S. V. Fomin, Элементы теории функций и функционального анализа, Nauka, Moscow, 1968. Translated as "Introductory real analysis", Dover, New York, 1975.

[Lanczos 1949] C. Lanczos, The variational principles of mechanics, University of Toronto Press, Toronto, 1949. Reprinted by Dover, New York, 1986.

[Lucchesi et al. 2006] M. Lucchesi, M. Šilhavý, and N. Zani, "A new class of equilibrated stress fields for no-tension bodies", J. Mech. Mater. Struct. 1 (2006), 503-539.

[Lucchesi et al. 2009] M. Lucchesi, M. Šilhavý, and N. Zani, "Equilibrated divergence measure stress tensor fields for heavy masonry bodies", Eur. J. Mech. A Solids 28:2 (2009), 223-232. 
[Marzocchi and Musesti 2003] A. Marzocchi and A. Musesti, "The Cauchy stress theorem for bodies with finite perimeter", Rend. Sem. Mat. Univ. Padova 109 (2003), 1-11.

[Mindlin 1964] R. D. Mindlin, "Micro-structure in linear elasticity", Arch. Ration. Mech. Anal. 16 (1964), 51-78.

[Noll 1959] W. Noll, "The foundations of classical mechanics in the light of recent advances in continuum mechanics", pp. 266-281 in The axiomatic method, with special reference to geometry and physics, edited by L. Henkin et al., North-Holland, Amsterdam, 1959. Reprinted in The foundations of continuum mechanics and thermodynamics: Selected papers of W. Noll, pp. 31-47, Springer, Berlin, 1974.

[Noll 1963] W. Noll, "La mécanique classique, basée sur un axiome d'objectivité", pp. 47-56 in La méthode axiomatique dans les mécaniques classiques et nouvelles (Paris, 1959), edited by J.-L. Destouches and F. Aeschlimann, Les Grands Problèmes des Sciences 13, Gauthier-Villars, Paris, 1963. Reprinted in The foundations of continuum mechanics and thermodynamics: Selected papers of W. Noll, pp. 135-144, Springer, Berlin, 1974.

[Noll 1973] W. Noll, "Lectures on the foundations of continuum mechanics and thermodynamics", Arch. Ration. Mech. Anal. 52 (1973), 62-92.

[Noll and Virga 1988] W. Noll and E. G. Virga, "Fit regions and functions of bounded variation", Arch. Ration. Mech. Anal. 102:1 (1988), 1-21.

[Noll and Virga 1990] W. Noll and E. G. Virga, "On edge interactions and surface tension", Arch. Ration. Mech. Anal. 111:1 (1990), 1-31.

[Podio-Guidugli 2004] P. Podio-Guidugli, "Examples of concentrated contact interactions in simple bodies", J. Elasticity 75:2 (2004), 167-186.

[Podio-Guidugli and Vianello 2010] P. Podio-Guidugli and M. Vianello, "Hypertractions and hyperstresses convey the same mechanical information", Contin. Mech. Therm. 22 (2010), 163-176.

[Šilhavý 1985] M. Šilhavý, "The existence of the flux vector and the divergence theorem for general Cauchy fluxes", Arch. Ration. Mech. Anal. 90:3 (1985), 195-212.

[Šilhavý 1990] M. Šilhavý, “On Cauchy's stress theorem”, Rend. Mat. Acc. Lincei (9) 1:3 (1990), 259-263.

[Šilhavý 1991] M. Šilhavý, "Cauchy's stress theorem and tensor fields with divergences in $L^{p}$ ", Arch. Ration. Mech. Anal. 116:3 (1991), 223-255.

[Šilhavý 2008] M. Šilhavý, "Cauchy's stress theorem for stresses represented by measures”, Contin. Mech. Therm. 20 (2008), 75-96.

[Timoshenko 1953] S. P. Timoshenko, History of strength of materials, McGraw-Hill, New York, 1953. Reprinted by Dover, New York, 1983.

[Toupin 1962] R. A. Toupin, "Elastic materials with couple-stresses", Arch. Ration. Mech. Anal. 11 (1962), 385-414.

[Toupin 1964] R. A. Toupin, “Theories of elasticity with couple-stress", Arch. Ration. Mech. Anal. 17 (1964), 85-112.

[Truesdell 1991] C. A. Truesdell, A first course in rational continuum mechanics, vol. 1, 2nd ed., Academic Press, Boston, 1991.

[Vol'pert and Hudjaev 1985] A. I. Vol'pert and S. I. Hudjaev, Analysis in classes of discontinuous functions and equations of mathematical physics, Mechanics: Analysis 8, Martinus Nijhoff, Dordrecht, 1985.

[Ziemer 1983] W. P. Ziemer, "Cauchy flux and sets of finite perimeter", Arch. Ration. Mech. Anal. 84:3 (1983), 189-201. 
[Ziemer 1989] W. P. Ziemer, Weakly differentiable functions: Sobolev spaces and functions of bounded variation, Graduate Texts in Mathematics 120, Springer, New York, 1989.

Received 16 Nov 2012. Revised 1 Apr 2013. Accepted 21 May 2013.

GianPIETRo Del Piero: dlpgpt@unife.it

Dipartimento di Ingegneria, Università di Ferrara, via Saragat 1, 44100 Ferrara, Italy 



\section{Guidelines for Authors}

Authors may submit manuscripts in PDF format on-line at the submission page.

Originality. Submission of a manuscript acknowledges that the manuscript is original and and is not, in whole or in part, published or under consideration for publication elsewhere. It is understood also that the manuscript will not be submitted elsewhere while under consideration for publication in this journal.

Language. Articles in MEMOCS are usually in English, but articles written in other languages are welcome.

Required items. A brief abstract of about 150 words or less must be included. It should be selfcontained and not make any reference to the bibliography. If the article is not in English, two versions of the abstract must be included, one in the language of the article and one in English. Also required are keywords and a Mathematics Subject Classification or a Physics and Astronomy Classification Scheme code for the article, and, for each author, postal address, affiliation (if appropriate), and email address if available. A home-page URL is optional.

Format. Authors are encouraged to use $\mathrm{IAT}_{\mathrm{E} X}$ and the standard amsart class, but submissions in other varieties of $\mathrm{T}_{\mathrm{E}} \mathrm{X}$, and exceptionally in other formats, are acceptable. Initial uploads should normally be in PDF format; after the refereeing process we will ask you to submit all source material.

References. Bibliographical references should be complete, including article titles and page ranges. All references in the bibliography should be cited in the text. The use of $\mathrm{BIBT}_{\mathrm{E}} \mathrm{X}$ is preferred but not required. Tags will be converted to the house format, however, for submission you may use the format of your choice. Links will be provided to all literature with known web locations and authors are encouraged to provide their own links in addition to those supplied in the editorial process.

Figures. Figures must be of publication quality. After acceptance, you will need to submit the original source files in vector graphics format for all diagrams in your manuscript: vector EPS or vector PDF files are the most useful.

Most drawing and graphing packages - Mathematica, Adobe Illustrator, Corel Draw, MATLAB, etc. - allow the user to save files in one of these formats. Make sure that what you are saving is vector graphics and not a bitmap. If you need help, please write to graphics@msp.org with as many details as you can about how your graphics were generated.

Bundle your figure files into a single archive (using zip, tar, rar or other format of your choice) and upload on the link you been provided at acceptance time. Each figure should be captioned and numbered so that it can float. Small figures occupying no more than three lines of vertical space can be kept in the text ("the curve looks like this:"). It is acceptable to submit a manuscript with all figures at the end, if their placement is specified in the text by means of comments such as "Place Figure 1 here". The same considerations apply to tables.

White Space. Forced line breaks or page breaks should not be inserted in the document. There is no point in your trying to optimize line and page breaks in the original manuscript. The manuscript will be reformatted to use the journal's preferred fonts and layout.

Proofs. Page proofs will be made available to authors (or to the designated corresponding author) at a Web site in PDF format. Failure to acknowledge the receipt of proofs or to return corrections within the requested deadline may cause publication to be postponed. 
Mathematics and Mechanics of Complex Systems vol. 2 no. 1

Delaminated thin elastic inclusions inside elastic bodies

Alexander M. Khludnev and Günter R. Leugering

Interfaces endowed with nonconstant surface energies

23 revisited with the d'Alembert-Lagrange principle

Henri Gouin

Particles for fluids: SPH versus vortex methods

Andrea Colagrossi, Giorgio Graziani and Mario

Pulvirenti

Nonclassical continua, pseudobalance, and the law of action 71 and reaction

Gianpietro Del Piero

MEMOCS is a journal of the International Research Center for the Mathematics and Mechanics of Complex Systems at the Università dell' Aquila, Italy.

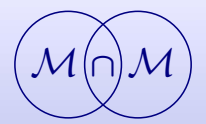

